\title{
Improved Frequency Domain Flutter Analysis Using Computational Fluid Dynamics
}

\author{
by \\ Ryan J. Beaubien \\ B. Eng. (Aerospace) \\ A thesis submitted to the Faculty of Graduate Studies and Research \\ in partial fulfillment of the requirements for the degree of \\ Master's of Applied Science in Aerospace Engineering \\ Ottawa-Carleton Institute for Mechanical and Aerospace Engineering \\ Department of Mechanical and Aerospace Engineering \\ Carleton University \\ Ottawa, Ontario, Canada
}

May 2006

(C) Copyright by Ryan J. Beaubien, 2006 


$\begin{array}{ll}\begin{array}{l}\text { Library and } \\ \text { Archives Canada }\end{array} & \begin{array}{l}\text { Bibliothèque et } \\ \text { Archives Canada }\end{array} \\ \begin{array}{l}\text { Published Heritage } \\ \text { Branch }\end{array} & \begin{array}{l}\text { Direction du } \\ \text { Patrimoine de l'édition }\end{array} \\ \begin{array}{l}\text { 395 Wellington Street } \\ \text { Ottawa ON K1A ON4 }\end{array} & \begin{array}{l}\text { 395, rue Wellington } \\ \text { Ottawa ON K1A ON4 } \\ \text { Canada }\end{array}\end{array}$

Your file Votre référence ISBN: 978-0-494-16452-5 Our file Notre référence ISBN: 978-0-494-16452-5

NOTICE:

The author has granted a nonexclusive license allowing Library and Archives Canada to reproduce, publish, archive, preserve, conserve, communicate to the public by telecommunication or on the Internet, loan, distribute and sell theses worldwide, for commercial or noncommercial purposes, in microform, paper, electronic and/or any other formats.

The author retains copyright ownership and moral rights in this thesis. Neither the thesis nor substantial extracts from it may be printed or otherwise reproduced without the author's permission.
AVIS:

L'auteur a accordé une licence non exclusive permettant à la Bibliothèque et Archives Canada de reproduire, publier, archiver, sauvegarder, conserver, transmettre au public par télécommunication ou par l'Internet, prêter, distribuer et vendre des thèses partout dans le monde, à des fins commerciales ou autres, sur support microforme, papier, électronique et/ou autres formats.

L'auteur conserve la propriété du droit d'auteur et des droits moraux qui protège cette thèse. $\mathrm{Ni}$ la thèse ni des extraits substantiels de celle-ci ne doivent être imprimés ou autrement reproduits sans son autorisation.
In compliance with the Canadian

Privacy Act some supporting forms may have been removed from this thesis.

While these forms may be included in the document page count, their removal does not represent any loss of content from the thesis.
Conformément à la loi canadienne sur la protection de la vie privée, quelques formulaires secondaires ont été enlevés de cette thèse.

Bien que ces formulaires aient inclus dans la pagination, il n'y aura aucun contenu manquant.

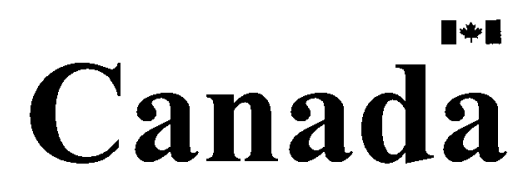




\section{Abstract}

The most widely used method for flutter certification is based on the linearized aerodynamic potential theory, specifically, the Doublet-Lattice Method (DLM). However, this method fails to accurately predict the aeroelastic behaviour in the transonic regime.

The present work investigates two different approaches which are able to capture the nonlinearities in the transonic regime: (1) performing a time marching simulation using Euler and Navier-Stokes equations; and (2) correcting the DLM aerodynamic data in a frequency domain analysis using Computational Fluid Dynamics (CFD) results. A flutter analysis of the AGARD 445.6 wing was subsequently conducted using the time marching and DLM correction approaches.

Various frequency domain correction techniques were considered within the present work resulting in the selection of an unsteady pressure matching method, based on a downwash weighting approach. Unsteady pressures employed for this technique were obtained from Euler and Navier-Stokes CFD simulations undergoing a rigid body pitching motion. 


\section{Acknowledgements}

I would like to thank Dr. Fred Nitzsche, my supervisor, and Dr. Daniel Feszty, my co-supervisor, for their suggestions and constant support during this research. I am deeply indebted to: Ihor Gegar (Bombardier Aerospace) for his guidance on structural and aerodynamic modelling; Dr. Roberto Gil Annes da Silva (Instituto de Aeronáutica e Espaço) for his assistance with the AIC correction procedure; Dr. Ken Badcock and Abdul Rampurawala (University of Glasgow) for providing the time marching code and assistance with CFD grid generation; and Johnny Cash for producing music that motivates the soul.

This thesis is dedicated to my family. 


\section{Contents}

Abstract $\quad$ iii

Acknowledgements $\quad$ iv

List of Tables viii

List of Figures $\quad$ ix

Nomenclature $\quad$ xi

1 Introduction 1

1.1 Description of Flutter . . . . . . . . . . . . . . 1

1.2 Historical Review of Flutter . . . . . . . . . . . . . . 2

1.3 Computational Analysis Techniques . . . . . . . . . . . . . 4

1.3.1 Frequency Domain Methods ............. 4

1.3.2 AIC Correction Methods . . . . . . . . . . . . . 7

1.3.3 Time Marching Methods . . . . . . . . . . . . . . 8

1.4 Basis and Overview of Current Work . . . . . . . . . . . 11

2 Frequency Domain Formulation $\quad 13$

2.1 Unsteady Aerodynamic Modelling . . . . . . . . . . . . . . 13

2.1.1 Overview of the Doublet-Lattice Method . . . . . . . . . . 14

2.2 Aeroelastic Model . . . . . . . . . . . . . . . 18

2.2.1 Aeroelastic Equations of Motion . . . . . . . . . . 18

2.2.2 Modal Approach . . . . . . . . . . . . . . . . 20 
2.2.3 Simple Harmonic Motion Approach . . . . . . . . . . . . . 22

2.2.4 Interconnection of the Structure with Aerodynamics . . . . . 22

2.3 Flutter Solution Techniques . . . . . . . . . . . . . 24

3 AIC Correction Formulation $\quad 26$

3.1 Overview of AIC Correction Methods . . . . . . . . . . . 26

3.1.1 Force Matching Methods . . . . . . . . . . . . . . . 26

3.1.2 Pressure Matching Methods . . . . . . . . . . . . . . 28

3.1.3 Dau-Garner Type Methods . . . . . . . . . . . . . . . . . . 31

3.1.4 Modal Aerodynamic Influence Coefficients Matrix Methods . . . . . . . . . . . . . . . . . 32

3.2 Derivation of Selected Correction Method . . . . . . . . . . . . . 33

3.2 .1 Theoretical Derivation . . . . . . . . . . . . . . 34

4 Time Marching Formulation $\quad 37$

4.1 Numerical Fluid Methods . . . . . . . . . . . . . . . . . . 37

$4.1 .1 \quad$ Euler Equations . . . . . . . . . . . . . . . . . . . 37

4.1 .2 Navier-Stokes Equations . . . . . . . . . . . . . . . . . 39

4.1 .3 Steady State Solver . . . . . . . . . . . . . . . . 42

$4.1 .4 \quad$ Unsteady Solver . . . . . . . . . . . . . . . . . . . . . 44

4.1 .5 Turbulence Model . . . . . . . . . . . . . . . . . 46

4.2 Structural Solver $\ldots \ldots \ldots \ldots \ldots \ldots \ldots$. . . . . . . . . 48

5 Test Cases 51

5.1 Validation of the Computational Procedure . . . . . . . . . . 51

$5.1 .1 \quad$ CFD Fluid Meshes . . . . . . . . . . . . . . . 52

5.1 .2 Steady Flow . . . . . . . . . . . . . . . . 53

5.1 .3 Unsteady Flow . . . . . . . . . . . . . . . . 54

5.2 Flutter Test Case . . . . . . . . . . . . . . . . . 56

5.2 .1 CFD Fluid Meshes . . . . . . . . . . . . . . . . . 59

$5.2 .2 \quad$ DLM Mesh . . . . . . . . . . . . . . 63 
5.2 .3 Structural Model . . . . . . . . . . . . . . . . . . 64

6 Results $\quad 68$

6.1 Uncorrected Frequency Domain Results . . . . . . . . . . . . 68

6.2 Time Marching Results . . . . . . . . . . . . . . . 70

6.2.1 Grid Density and Fluid Model Effect on Flutter Speed . . . . 70

6.2 .2 Flutter Boundaries ................ 72

6.3 AIC Correction Method Results . . . . . . . . . . . . . . 79

6.3.1 Present Work Results . . . . . . . . . . . . . 80

6.3.2 Referenced Results ................ 83

6.4 Conclusions .......................... 84

6.5 Future Works . . . . . . . . . . . . . 85

$\begin{array}{lr}\text { References } & 87\end{array}$ 


\section{List of Tables}

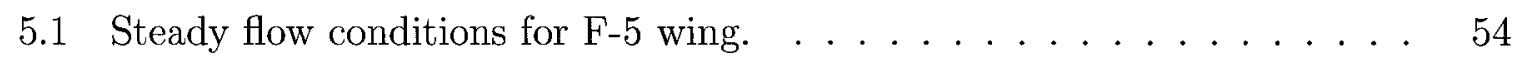

5.2 Unsteady flow conditions for $\mathrm{F}-5$ wing. . . . . . . . . . . . 54

5.3 Flow conditions for AGARD 445.6 wing flutter analysis. . . . . . . . 60

5.4 Comparison of modal frequencies for AGARD 445.6 wing. . . . . . . . 64

6.1 Comparison of flutter speed coefficients at Mach 0.96 for various grids. . 72

6.2 Reduced frequencies of the CFD simulations used in the AIC correction method. . . . . . . . . . . . . . . 79

6.3 Computational time required to calculate each flutter point. . . . . . . 84 


\section{List of Figures}

2.1 Lifting surface idealization in the DLM. . . . . . . . . . . . 15

2.2 DLM wing and element coordinates. . . . . . . . . . . 16

$5.1 \quad$ F-5 wing fluid mesh. . . . . . . . . . . . . . . 52

5.2 Steady $C_{p}$ over F-5 upper wing surface at $M_{\infty}=0.95 \ldots \ldots \ldots \ldots 53$

5.3 Steady $C_{p}$ and $\Delta C_{p}$ over F-5 wing at $M_{\infty}=0.95 \ldots \ldots \ldots \ldots \ldots$

5.4 Steady state convergence for F-5 wing at $M_{\infty}=0.95 \ldots \ldots \ldots \ldots$

5.5 Real and imaginary $C_{p}$ over F-5 wing at $M_{\infty}=0.95$ for 2 and 3 cycles. . $\quad 57$

5.6 Real and imaginary $C_{p}$ over F-5 wing at $M_{\infty}=0.95 \ldots \ldots \ldots \ldots$

5.7 Unsteady $\Delta C_{p}$ over F-5 wing at $M_{\infty}=0.95 \ldots \ldots \ldots \ldots \ldots$

5.8 Medium density AGARD 445.6 CFD meshes. . . . . . . . . . . . 61

5.9 Steady $C_{p}$ over AGARD 445.6 upper wing surface at $M_{\infty}=0.96 . \quad \ldots \quad 62$

5.10 Steady state convergence for medium density AGARD 445.6 wing meshes

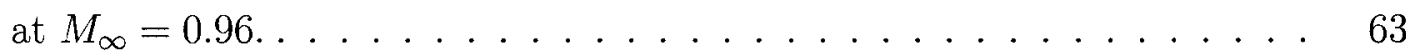

5.11 AGARD 445.6 structural model. . . . . . . . . . . . . . . . . 66

5.12 Calculated AGARD 445.6 wing mode shapes. . . . . . . . . . . 67

6.1 Uncorrected frequency domain flutter boundaries for AGARD 445.6 wing. 69

6.2 Uncorrected frequency domain $\mathrm{V}-\mathrm{f}$ and $\mathrm{V}-\mathrm{g}$ plots at $M_{\infty}=0.678 . \ldots 71$

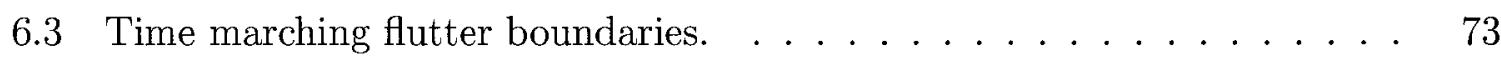

6.4 Time marching responses at $M_{\infty}=0.678 \ldots \ldots \ldots \ldots \ldots \ldots$

6.5 Time marching responses at $M_{\infty}=0.901 \ldots \ldots \ldots \ldots \ldots \ldots$

6.6 Time marching responses at $M_{\infty}=0.960 \ldots \ldots \ldots \ldots \ldots$

6.7 Time marching responses at $M_{\infty}=0.990 \ldots \ldots \ldots \ldots \ldots \ldots$ 
6.8 Time marching responses at $M_{\infty}=1.072 \ldots \ldots \ldots$. . . . . . 78

6.9 Unsteady $\Delta C_{p}$ over AGARD 445.6 wing at $M_{\infty}=0.678 \ldots \ldots$. . . . 81

6.10 AIC corrected frequency domain V-f and V-g plots at $M_{\infty}=0.678$. . . $\quad 82$

6.11 Flutter boundaries for AGARD 445.6 wing. . . . . . . . . . . . . 83 


\section{Nomenclature}

$\Delta \alpha$

$\Delta \xi$

$\Delta C_{p}$

$\Delta p$

$\mathrm{w}$

K

$[\Phi]$

$[A I C]$

$[D]$

$[F]$

$[G]$

$[H]$

$[K]$

$[M]$

$[Q]$
Amplitude of dynamic angle of attack

Body element length

Lifting pressure coefficient, $\Delta C_{p}=\left(p_{\text {lower }}-p_{\text {upper }}\right) / q_{\infty}$

Lifting pressure

Dimensionless normal velocity, $\mathrm{w}=W / U_{\infty}$

Kernel function

Modal transformation matrix, composed of mode shape vectors

Aerodynamic influence coefficients matrix

Influence matrix relating normalwash to lifting pressure

Substantial derivative operator

Spline matrix operator

Aerodynamic transfer function matrix

Structural stiffness matrix

Structural mass matrix

Aerodynamic loads influence coefficients matrix 
Integration matrix

Weighting matrix operator

Total aerodynamic force vector

$\left\{L_{a}\right\} \quad$ Induced aerodynamic force vector

$\left\{L_{e}\right\} \quad$ External aerodynamic force vector

$\{q\}$

Generalized coordinate vector

$\{\overline{\mathrm{w}}\}$

Dimensionless normal velocity vector divided by $\Delta \alpha$

$\{\mathrm{w}\}$

Dimensionless normal velocity vector

$\{u\}$

Structural displacement vector

$\mu$

Reference viscosity

$\mu_{T}$

Eddy viscosity

$\nu$

Poisson's ratio

$\Omega$

Angular velocity

$\omega$

Oscillation frequency associated with harmonic motion

$\operatorname{Re}(), \operatorname{Im}()$

Real and imaginary parts of a complex number

$\rho$

Density

$\sigma$

Lateral coordinate in the plane of the lifting surface

$\tau=U_{\infty} t / c$

Non-dimensional time

$\tau_{i j}$

Stress tensor

$\varphi_{j}^{0}$

Blending function

xii 


\begin{tabular}{|c|c|}
\hline$\xi, \bar{\eta}, 0$ & Coordinates of a point pressure doublet in element coordinates \\
\hline$\xi, \eta, \zeta$ & Coordinates of a point pressure doublet \\
\hline$\xi_{1 / 4}$ & One-quarter chord point of lifting surface element \\
\hline$b$ & Reference length (root semi-chord) \\
\hline$c_{r}$ & Wing root chord \\
\hline$C D_{k \omega}$ & Cross-diffusion term for the $k-\omega$ turbulence model \\
\hline$e$ & Semi-width of lifting surface element \\
\hline$E_{1}$ & Longitudinal elastic modulus \\
\hline$E_{2}$ & Lateral elastic modulus \\
\hline$F_{1}$ & SST coefficient blending function \\
\hline$F_{2}$ & SST closure forcing function \\
\hline$G$ & Shear modulus \\
\hline$g$ & Structural damping \\
\hline$H$ & Enthalpy \\
\hline$h$ & Displacement mode shape vector \\
\hline$i$ & Imaginary number, $i=\sqrt{-1}$ \\
\hline$k$ & $\begin{array}{l}\text { Reduced frequency, } k=\omega b / U_{\infty} \text { or turbulent kinetic energy } \\
\text { (Chapter } 5 \text { ) }\end{array}$ \\
\hline$M_{\infty}$ & Freestream Mach number \\
\hline$n l$ & Nonlinear \\
\hline
\end{tabular}

xiii 


\begin{tabular}{|c|c|}
\hline$P_{\gamma_{T}}$ & Turbulent Prandtl number \\
\hline$P_{\gamma}$ & Prandtl number \\
\hline$q_{\infty}$ & Dynamic pressure, $q_{\infty}=\frac{1}{2} \rho U_{\infty}^{2}$ \\
\hline$q_{x}, q_{y}, q_{z}$ & Heat flows \\
\hline$s$ & Laplace variable, $s=i \omega$ \\
\hline$T$ & Static temperature \\
\hline$t$ & Time \\
\hline$U, V, W$ & Contravariant velocities \\
\hline$u, v, w$ & Cartesian velocities \\
\hline$U_{\infty}$ & Freestream velocity \\
\hline$U_{F}$ & Flutter speed coefficient \\
\hline$W$ & Normal velocity at surface \\
\hline$x, \bar{y}, \bar{z}$ & $\begin{array}{l}\text { Coordinates of a general field point given in terms of a coordi- } \\
\text { nate system centred on and rotated into the plane of a lifting } \\
\text { surface element, i.e. element coordinate system }\end{array}$ \\
\hline$x, y, z$ & Cartesian coordinates of a general field point \\
\hline$y$ & Wall distance \\
\hline $\mathbf{F}, \mathbf{G}, \mathbf{H}$ & Flux vectors \\
\hline $\mathbf{F}^{i}, \mathbf{G}^{i}, \mathbf{H}^{i}$ & Inviscid viscous flux vectors \\
\hline $\mathbf{F}^{v}, \mathbf{G}^{v}, \mathbf{H}^{v}$ & Diffusive viscous flux vectors \\
\hline $\mathrm{Q}$ & Heat flow vector \\
\hline
\end{tabular}

xiv 
$\mathbf{R}$

W

AIC

BILU

CFD

CSD

CVT

DLM

$\mathrm{LCO}$

MAIC

RANS

Re

TFI

TSD
Residual vector

Solution vector

Aerodynamic Influence Coefficients

Block Incomplete Lower Upper

Computational Fluid Dynamics

Computational Structural Dynamics

Constant Volume Tetrahedron

Doublet-Lattice Method

Limit Cycle Oscillation

Modal Aerodynamic Influence Coefficients

Reynolds Averaged Navier-Stokes

Reynolds number

Transfinite Interpolation of Displacements

Transonic Small Disturbance 


\section{Chapter 1}

\section{Introduction}

\subsection{Description of Flutter}

Flutter, the dynamic instability of an elastic body in an airstream, is commonly encountered on bodies subjected to large lateral aerodynamic loads such as on aircraft wings, tails and control surfaces [1]. No aircraft is perfectly rigid due to weight constraints and its flexibility can interact with the airstream, sometimes creating self-sustaining vibrations. For example, an aircraft wing is relatively flexible and can be observed to bend and twist under the influence of air loads in flight. If this motion occurs in a periodic manner under certain conditions, the dynamic loads may begin to augment the elastic motion of the wing. As the amplitude of the motion increases, the loads on the structure also increase, eventually causing the structure to catastrophically fail. The speed at which this phenomenon occurs is called the flutter speed. Flights at speeds above and below the flutter speed yield unstable and stable conditions, respectively. 
Modern aircraft are subject to many kinds of flutter phenomena. The classical type of flutter is associated with potential flow and usually, but not necessarily, involves the coupling of two or more degrees of freedom - wing bending and torsion being the most common. The nonclassical type may involve separated flows, stalling conditions and time-lag effects between the aerodynamic forces and the motion of the structure [1]. The simulation of the classical type of flutter is the subject of this thesis.

\subsection{Historical Review of Flutter}

In 1916, the first recorded flutter incident occurred on a Handley Page O/400 twin engine biplane bomber. The bomber experienced violent self-excited antisymmetric oscillations of the fuselage and tail. The problem was eliminated by increasing the stiffness of the fuselage and tail structures.

In the period from 1918 to the 1930s, a systematic flutter study was conducted for the van Berkel monoplane, a seaplane for reconnaissance. The flutter mechanism was a coupling of the vertical bending of the wing with the motion of the ailerons and was negated by moving the aileron to balance the wing mass [2].

In the 1930s, with the availability of higher performance engines, flutter began to be recognized as a critical safety concern. The solution of increasing structural stiffness was not always feasible due to weight considerations. Experiments conducted during this decade revealed that altering the mass distribution of the aircraft was as effective as modifying the flight velocity at which flutter occurred as was increasing 
the structural stiffness [3].

During World War II, the importance of flutter in aircraft design was increased as designers incorporated new structural materials and more powerful engines. In 1945, 146 flutter incidents occurred in Germany, resulting in 24 crashes [2]. All of these incidents involved flutter of the control surfaces or the auxiliary controls. Only 54 flutter incidents were recorded during the 10 years following the war. This decrease can be attributed to advances in computational methods that complemented improvement in experimental techniques for flutter models, ground resonance testing and flight flutter testing [2], [4].

From 1947 to 1956 , several incidents of flutter were recorded which involved the carriage of external stores, such as external fuel tanks or missiles, or pylon mounted engines [2]. This problem is still significant today as most aircraft are capable of several store configurations; certain combinations of external stores carried by the American F-16, F-18 and F-111 aircraft produce a limit cycle oscillation (LCO). These oscillations are characterized by sustained sinusoidal oscillations of limited amplitude. Although LCOs may not be destructive, they may be uncomfortable for the pilot, may decrease the life of the aircraft and may negatively affect the precision of the air-to-air missile systems. In 1999, unexpected instabilities arose for the Mitsubishi F-2A/B fighter which delayed the program for over 9 months [5]. The problem was eventually linked to the presence of stores. Stores were also responsible for a $30 \%$ reduction in 
the flight envelope for the Royal Australian Air Force F-18 [6].

Other recent examples not involving LCOs include the Taiwan IDF fighter which crashed due to flutter of the horizontal tail during a high dynamic pressure flight test in 1992, ultimately leading to the cancellation of the project. Also in 1992, a prototype of the American F-22 Raptor crashed in a flutter related accident [7]. In 1997, an American F-117 Stealth fighter crashed due to aileron/flaperon flutter on a primary lifting surface [8].

Today, few catastrophic flutter incidents occur due to the improvements in flight flutter test technique, instrumentation and response data analysis [9]. However, flutter problems still arise regularly as illustrated by the examples cited above. These problems delay projects and incur substantial additional expense. Flutter testing is still considered hazardous due to the violent nature of the onset of flutter. Accurately predicting the flutter boundary in the transonic regime using computational methods would result in a shorter and less expensive flight testing program as the pilots and engineers would have greater confidence in the predictions.

\subsection{Computational Analysis Techniques}

\subsubsection{Frequency Domain Methods}

The aeronautical industry mainly employs commercial codes such as MSC/NASTRAN and ZAERO for flutter prediction. These codes perform a modal eigenvalue analysis using a structural dynamic solver and unsteady aerodynamics based on linearized 
aerodynamic potential theories. The aerodynamic analysis, like the structural analysis, is based on a finite element approach. A brief summary of the flutter solution procedure follows; refer to Chapter 2 for the detailed derivation.

The aeroelastic response of a body in an airstream is a result of the mutual interaction of: (1) inertial and elastic structural forces; (2) aerodynamic forces induced by static or dynamic deformation of the structure; and (3) external disturbance forces [10]. The equations of motion for the body are linearized and transformed into the frequency domain as the classical approach assumes that the structure undergoes low amplitude elastic harmonic motion. The aerodynamic forces are computed which are related to the structural deformations using an aerodynamic influence coefficients (AIC) matrix. The roots for the system of homogeneous equations of motion are determined using various eigenvalue solution techniques. A root corresponding to a decaying or stable condition is considered to be below the flutter speed. A root corresponding to a divergent oscillation with positive damping is assumed to be beyond the flutter speed. The root corresponding to neutral stability gives the flutter speed [3].

AICs reduce development costs and are therefore beneficial in a production flutter environment. Once the AIC matrices have been generated for a specific planform, an unlimited number of aeroelastic analyses can be performed in which only the inertial and stiffness properties are altered as the AICs are independent of the aircraft 
vibration modes shapes [11].

Several subsonic and supersonic aerodynamic theories are able to accurately predict the flow in the Mach range of $0.0<M_{\infty}<0.6$ and $M_{\infty}>1.1$. Common subsonic theories used in production flutter analysis include the modified strip theory, DoubletLattice Method (DLM) and ZONA6. Common supersonic theories include the Mach box method, Piston theory and ZONA51. Strip theory was the primary subsonic unsteady aerodynamic theory used from the early 1940 s to the mid 1960s.

In 1966, Reference [12] proposed modifying strip theory to account for finite span effects. The resultant method was referred to as modified strip theory. Coupled with the solution procedure outlined above, modified strip theory formed the basis for production flutter analyses in the late 1960s [13].

Today, industry primarily utilizes the DLM for calculating unsteady aerodynamic loads. During the late 1960s, this unsteady panel aerodynamic method was developed by Albano and Rodden [14] and refined by the Douglas Aircraft Company [15]. The DLM has been in use for over 30 years and is the standard by which new unsteady aerodynamic codes are evaluated. It is anticipated that the DLM will be used for many years to come [13].

Since the governing equations over which the unsteady aerodynamic methods were developed are based on a linearized unsteady potential flow hypothesis, the application of these methods are limited to purely subsonic or supersonic flows [16]. 
This is unfortunate as most passenger aircraft cruise at transonic speeds [3]. These methods are unable to capture the location and magnitude of local shock waves and the associated shock wave-boundary layer interactions on wing surfaces in the transonic regime. The prediction of these phenomena are crucial for assessing the aeroelastic behaviour of a wing in transonic flow as they are the source for several nonlinear aeroelastic effects, such as the transonic dip and LCOs.

\subsubsection{AIC Correction Methods}

Several procedures to solve the transonic aeroelastic problem have been developed over the past 40 years [17]. These procedures are commonly referred to as combined procedures, as mixed procedures or as semi-empirical corrections. Measured or computed data is related to the unsteady linear aerodynamic model so as to account for nonlinear effects unpredicted by the linearized potential-based equations of the fluid flow [16].

These corrections can be performed by the pre- or post-multiplication, addition or whole replacement of the AIC matrix. This approach is adequate for engineering applications as it is less expensive than the direct use of computational fluid dynamic techniques. The correction techniques, which have been applied to unsteady loading calculations for aeroelastic analysis, are classified in four major classes: (1) force matching methods [12], [18], [19], [20], [21]; (2) pressure matching methods [16], [22], [23], [24], [25], [26], [27]; (3) Dau-Garner type methods [28], [29], [30], [31]; and (4) 
modal aerodynamic influence coefficients (MAIC) matrix replacement [32], [33].

The first procedure matches reference nonlinear forces and moments which may be obtained from experiments or Computational Fluid Dynamics (CFD) simulations. In this case, nonlinearities such as pressure jumps due to shock waves and viscous effects are embedded in the reference quantities. The second procedure matches reference nonlinear pressures, thereby maintaining the same nonlinearities as the first method. The third procedure employs steady nonlinear information and semi-empirical relations in order to compute the unsteadiness of the resulting nonlinear corrected pressures. The fourth procedure generates a MAIC matrix which is referred to measured or computed nonlinear pressures or loading due to given modal displacements of the lifting surface. This new matrix is substituted in the aeroelastic equations of motion where the generalized unsteady aerodynamic forces are related to the associated modal displacements of the lifting surface or downwash mode shapes. Commonly employed methods for each class will be discussed in Chapter 3.

\subsubsection{Time Marching Methods}

A feasible solution to the transonic aeroclastic problem is to complete a time marching analysis where a CFD solver is coupled with a Computational Structural Dynamics (CSD) solver. In these simulations, the structure is given an initial velocity in one of the dominant modes and the subsequent time evolution of the modal response is calculated to see whether it grows or decays. The flow is usually simulated by solving 
the Euler or Reynolds' Averaged Navier-Stokes (RANS) equations. AERO-F and AERO-S and CFL3D are examples of CSD/CFD codes which have been successfully used to simulate flutter on a complete aircraft [34].

In fluid-structure interaction problems, the fluid solution is usually computed on an Eulerian coordinate system, whereas the structural component is solved in a Lagrangian system. There are three classes of time marching codes: (1) a loosely coupled code; (2) a direct approach code; and (3) a simultaneously coupled code.

A loosely coupled code will solve the fluid and structural systems utilizing two separate codes. Load and deformation information is transferred between the separate codes using an interfacing system. The modal approach is used in order to reduce computation costs. The advantage of this approach is that well established fluid and structural codes may be employed. The disadvantage is that errors can be introduced during the transformation of information and sequencing between the codes [3]. In the direct approach, the aerodynamic forces are calculated by the CFD code and are mapped onto the structural nodes. The CSD code calculates the structural response which is interpolated back onto the CFD grid. The CFD code then again calculates the aerodynamic forces and this continues until a defined convergence criteria is met. The direct approach is more accurate than the loosely coupled modal approach. The disadvantage is that a high computation cost is occurred inputting and outputting the CFD and CSD responses. The final approach is to combine the 
fluid and structural solvers into a single code, referred to as a simultaneously coupled code. The computational time is reduced at the cost of increasing the complexity of the code [3]. A brief history of the development of time marching methods follows; refer to Reference [3] for a detailed history.

In 1982, Reference [35] created a loosely coupled aeroelastic code, XTRANS3S. This was one of the first nonlinear transonic flutter analyses where the fluid was modelled using the Transonic Small Disturbance (TSD) equation and the structural deformation was represented by the modes of the structure. Reference [36] further developed the TSD technique resulting in a new code, the Computational Aeroelasticity Program-TSD (CAP-TSD). Reference [37] used CFL3D, a modified RANS code, to calculate wing flutter using Navier-Stokes aerodynamics on the AGARD 445.6 wing [3]. In 1993, Reference [38] introduced the method of direct coupling of a plate FE model with an Euler aerodynamic solver. In 1999, Reference [4] created a loosely coupled aeroelastic code using a transformation scheme based on the Constant Volume Tetrahedron (CVT) technique. Flutter calculations were performed on the AGARD 445.6 wing and the MDO wing. Reference [39] performed an aeroelastic simulation of an F-16 aircraft using a direct approach. In 2003, Reference [34] applied the three field Arbitrary Lagrangian-Euler formulation of the Euler and Navier-Stokes equations over an F-16 aircraft using a detailed structural FE model. 


\subsection{Basis and Overview of Current Work}

The aircraft industry requires a robust and efficient aerodynamic tool for use in transonic flutter analyses. Presently, a subsonic linearized discrete lifting surface theory, the DLM, is employed but it cannot capture the flow nonlinearities in the transonic regime. As discussed above, these nonlinearities can be captured using two different approaches: (1) correcting the DLM aerodynamic data in a frequency domain analysis using CFD or experimental results; or (2) performing a time marching simulation using Euler or Navier-Stokes equations.

The objective of the present work is to compare flutter boundaries of a finite wing using both uncorrected and corrected DLM aerodynamics in a frequency domain analysis with a time marching analysis using Euler and Navier-Stokes equations. A single AIC correction procedure will be analyzed that matches reference unsteady pressures obtained from CFD simulations undergoing a rigid body pitching motion.

Uncorrected frequency domain results will be obtained using DLM aerodynamics in MSC/NASTRAN and KEDLMPL. KEDLMPL, developed at the Instituto de Aeronáutica e Espaço in Brazil, is a subsonic DLM code which performs a KE-flutter analysis using the AIC matrix approach. The AIC correction procedure will be implemented into this code as current versions of MSC/NASTRAN will not allow direct access to the AIC matrices. 
The time marching simulations will be performed using the PMB (Parallel MultiBlock) code developed at the University of Glasgow. This implicit code features a finite-volume Euler and RANS CFD solver with a proven capability of accurately capturing transonic effects [4], [40].

Chapter 2 will present the theoretical background of the Doublet-Lattice method and the derivation of the frequency domain flutter solution procedure. Chapter 3 will derive the AIC correction procedure. The time marching procedure using Euler and Navier-Stokes equations will be detailed in Chapter 4. The test case, including structural and fluid models, will be defined in Chapter 5. Results and suggestions for future works will be presented in Chapter 6 . 


\section{Chapter 2}

\section{Frequency Domain Formulation}

\subsection{Unsteady Aerodynamic Modelling}

The classic approach in solving the unsteady compressible flow problem is through the use of lifting surface theory. Basic lifting surface theory assumes that flow is inviscid, isentropic, subsonic, and contains no flow separation. The thickness of the surface is neglected and the angle of attack is small so such that the small-disturbance potential flow approach may be used to linearize the mixed boundary value problem. The compressibility effect is taken into account in the aerodynamic governing equation using the Prandtl-Glauert transformation [1], [41], [42].

Based on a finite element approach, the DLM achieved wide acceptance due to its simplicity, accuracy and versatility. This section presents an overview of the DLM for use in flutter analyses. A brief review of the DLM follows; for a detailed derivation, refer to References [11], [14] and [15]. 


\subsubsection{Overview of the Doublet-Lattice Method}

The linearized formulation of the oscillatory subsonic lifting surface theory relates the normal velocity, $W$, at the surface

$$
W=U_{\infty} \operatorname{Re}\left(\mathrm{w} e^{i \omega t}\right)
$$

to the pressure difference across the surface

$$
\Delta p=\frac{1}{2} U_{\infty}^{2} \operatorname{Re}\left(\Delta C_{p} e^{i \omega t}\right)
$$

by a singular integral equation, transformed into the reduced frequency domain,

$$
\mathrm{w}(x, y, z)=\frac{1}{8 \pi} \iint \mathrm{K}\left(x-\xi, y-\eta, z-\zeta, k, M_{\infty}\right) \Delta C_{p} \mathrm{~d} \xi \mathrm{d} \sigma
$$

where $\xi$ is the streamwise coordinate, $\sigma$ is the tangential spanwise coordinate (as shown in Fig. 2.1), $k=\omega b / U_{\infty}$ is the reduced frequency, $\omega$ is the frequency of oscillation, $b$ is the root semi-chord, $U_{\infty}$ is the freestream speed and $\Delta C_{p}$ is the dimensionless lifting pressure coefficient. The kernel, $\mathrm{K}$, is the normalwash (or downwash) at a point $x, y, z$ induced by a pressure doublet of unit strength located at $\xi, \eta, \zeta$ and is given in Reference [15].

The lifting surface is divided into a grid of trapezoidal elements over which the lifting pressure is assumed constant. Refer to Fig. 2.1. Element coordinates are used to aid in performing the double integral of the kernel over each element. Let $x, \bar{y}, \bar{z}$ and $\xi, \bar{\eta}, 0$ be coordinates of the receiving and sending points, respectively, in a coordinate 


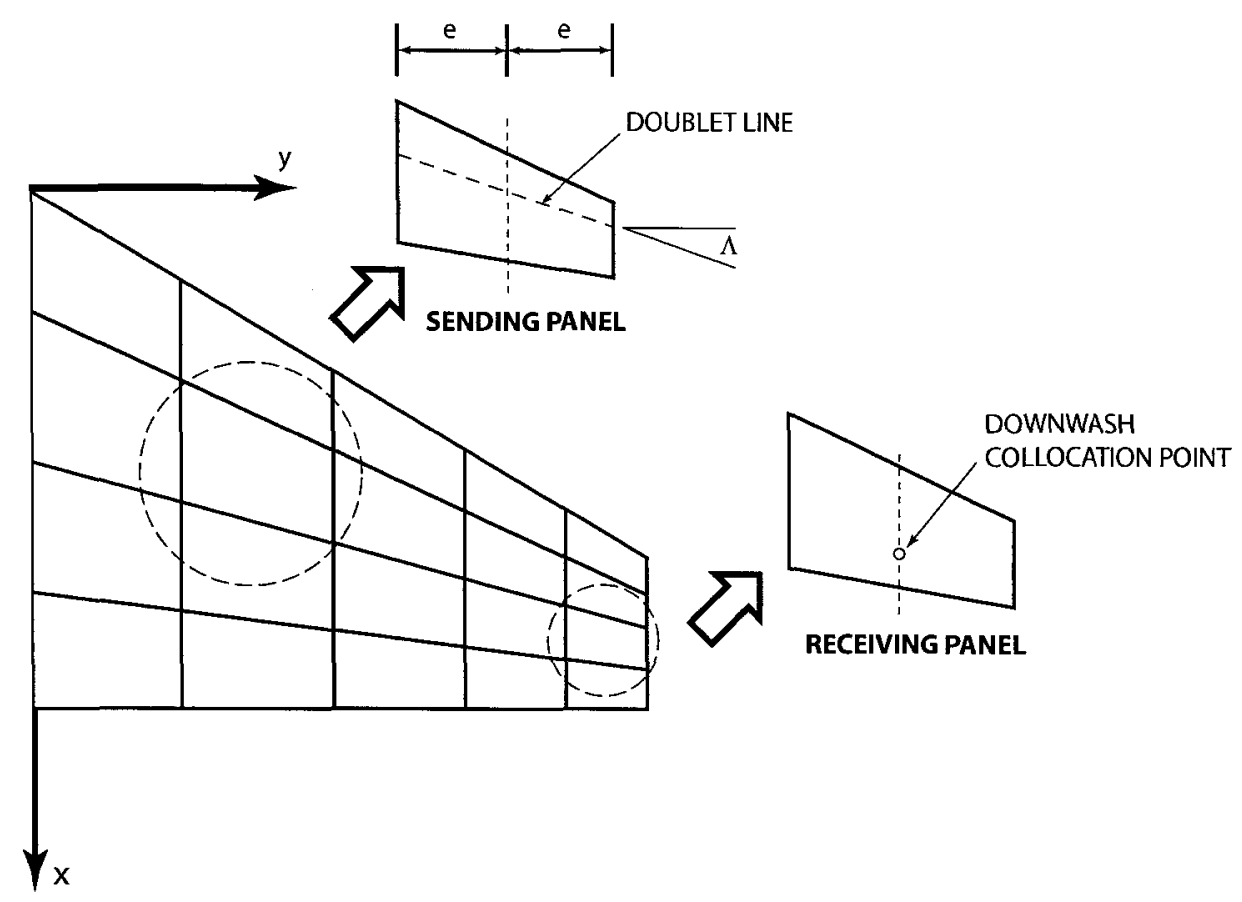

Figure 2.1: Lifting surface idealization in the DLM.

system centred on and rotated into the plane of the element. Refer to Fig. 2.2. The double integral over the element is then performed in the $\xi$ - and $\bar{\eta}$-directions. The integration of $\mathrm{K}$ in the streamwise direction $(\xi)$ is performed approximately by concentrating the value of the integrand at the one-quarter chord point, $\xi_{1 / 4}$. Eq. (2.3) becomes

$$
\mathrm{w}(x, y, z)=\sum_{j} \Delta C_{p_{j}} \frac{\Delta \xi_{j}}{8 \pi} \int_{-e_{j}}^{e_{j}} \mathrm{~K}\left(x-\xi_{1 / 4}, \bar{y}-\bar{\eta}, \bar{z}, k, M_{\infty}\right) \mathrm{d} \bar{\eta}
$$

where $e$ is the semi-width of the element. The result is an unsteady horseshoe vortex whose bound portion lies along the one-quarter chord line of the element. The change in variable from $\sigma$ to $\bar{\eta}$ indicates that element coordinates are to be used.

In Eq. (2.4), the downwash boundary condition $\mathrm{w}(x, y, z)$ is known and the lifting 


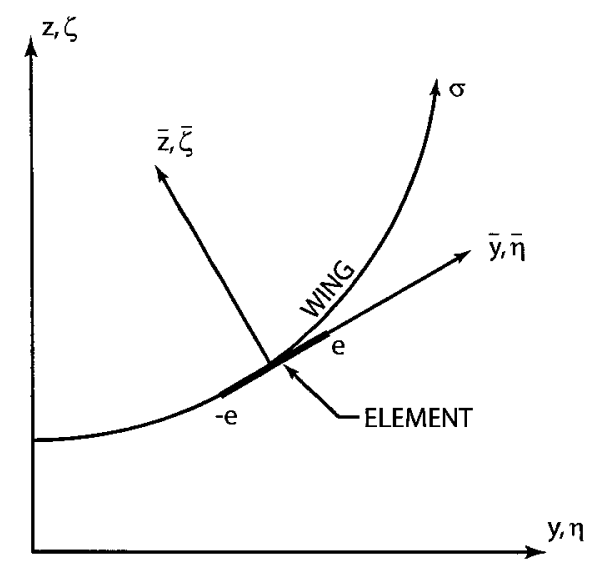

Figure 2.2: DLM wing and element coordinates.

pressure, $\Delta C_{p_{j}}$, over each element is unknown. A set of linear algebraic equations may be formed if the downwash is satisfied for each element. Eq. (2.4) can be then written in matrix form

$$
\{\mathrm{w}\}=[D]\left\{\Delta C_{p}\right\}
$$

where a typical element of $[D], D_{i j}$, is

$$
D_{i j}=\frac{\Delta \xi_{j}}{8 \pi} \int_{e_{j}}^{e_{j}} \mathrm{~K}\left(x_{i}-\xi_{1 / 4_{i}}, \bar{y}_{i}-\bar{\eta}, \bar{z}_{i}, k, M_{\infty}\right) \mathrm{d} \bar{\eta}
$$

The term $D_{i j}$ is the downwash factor at $x_{i}, \overline{y_{i}}, \overline{z_{i}}$ due to element $j$. This term is broken down into a steady part and an unsteady increment and the solution must satisfy two boundary conditions, the so-called Kutta condition and the flow tangency condition. The Kutta condition states that the pressure difference at the trailing edge of a thin lifting surface must be zero. The flow tangency condition states that for an inviscid flow, the velocity vector must be tangent to the surface of the streamlined body [42]. There is one control or receiving point for each element and the latter 
boundary condition is satisfied at each of these points. The control point is centred spanwise on the three-quarter chord line of the element.

The inverse of the matrix $[D]$ multiplied by the downwash vector, $\{w\}$, yields the pressure distribution. The resulting inverse matrix is the AIC matrix which is a function of the reduced frequency and is related to the pressure distribution by

$$
\left\{\Delta C_{p}(i k)\right\}=[A I C(i k)]\{\mathrm{w}(i k)\}
$$

The coefficients of the AIC matrix may be interpreted as rates of pressure variation due to a given displacement amplitude input associated with the boundary conditions. The determination of the pressure coefficient vector in Eq. (2.7) is performed from the known downwash which is related to the amplitude of the pitch and plunge motion at each element. The substantial derivative of a given displacement mode is composed of a derivative of the normal direction displacement plus the associated velocity scaled by the undisturbed speed. In a small disturbance sense, this quantity represents an angle of attack [16]. Therefore, from the boundary conditions for those small perturbations, the relationship between the normalwash and a solid boundary displacement is written as

$$
\{\mathrm{w}(i k)\}=\frac{\partial h(x, y, 0)}{\partial x}+i k h(x, y, 0)=[F(i k)]\{h(x, y, 0)\}
$$

The substantial derivative applied to a given modal displacement vector, $\{h\}$, is denoted by the matrix operator $[F(i k)]$. The resulting aerodynamic loading vector, $\left\{L_{a}(i k)\right\}$ may be expressed as the multiplication of the pressures by an integration 
matrix, $[S]$, which is constructed from the geometrical characteristics of the panel elements [16]. The resulting final expression for the unsteady loading over the lifting surface is given by

$$
\left\{L_{a}(i k)\right\}=q_{\infty}[S][A I C(i k)][F(i k)]\{h\}
$$

where $q_{\infty}$ is the dynamic pressure and the matrix $[F(i k)]$ is given by

$$
[F(i k)](\cdot)=\left[\frac{\partial(\cdot)}{\partial x}+i k(\cdot)\right]
$$

\section{$2.2 \quad$ Aeroelastic Model}

\subsubsection{Aeroelastic Equations of Motion}

The equation of motion for a structure in an airstream can be represented as an equilibrium between the structural and aerodynamic forces as

$$
[M]\{\ddot{u}(t)\}+[K]\{u(t)\}=\{L(u(t), \dot{u}(t))\}
$$

where $[M]$ and $[K]$ are the structural mass and stiffness matrices, respectively, and are usually obtained from a finite element model of the body. The structural deformation is denoted as $\{u\}$. The aerodynamic forces applied on the structure are denoted as $\{L\}$. The terms $[M]\{\ddot{u}(t)\}$ and $[K]\{u(t)\}$ are referred to as the inertial and elastic structural forces, respectively. The structural damping matrix is excluded from Eq. (2.11) for illustration purposes. Structural damping, typically small for an aircraft, will appear later in the derivation as artificial damping. The aerodynamic 
forces can be split into two components: (1) the aerodynamic forces induced by the structural deformation, $\left\{L_{a}(u(t), \dot{u}(t))\right\}$; and (2) the external forces, $\left\{L_{e}(t)\right\}$. Typical examples of external forces are gust loads, atmospheric turbulence, store ejection forces and pilot induced control surface aerodynamic forces [10]. These forces are considered for dynamic system response investigations. The induced aerodynamic loads may be generated by unsteady aerodynamic theories. Since these loads depend on the structural deformation, the relationship can be interpreted as an aerodynamic feedback system [16]. Eq. (2.11) is rewritten as

$$
[M]\{\ddot{u}(t)\}+[K]\{u(t)\}-\left\{L_{a}(u(t), \dot{u}(t))\right\}=\left\{L_{e}(t)\right\}
$$

The investigation of the dynamic stability of Eq. (2.12) is known as the flutter problem [16]. Flutter analysis usually involves the search of the structural stability boundary of an aircraft structure in terms of its flight speed and altitude or the corresponding dynamic pressure. This boundary is determined by examining the decay or growth of the structural response with respect to the flight speed. The structural dynamic model, which is the left hand side of Eq. (2.12), is generally linear as amplitudes of deformations are small for aeroelastic phenomena [1]. When the aerodynamic response may be assumed linear with respect to the structural deformation, Eq. (2.12) is a linear system. The flutter boundary is determined by solving the complex eigenvalues of this system. 
The solution of the eigenvalue problem requires the homogeneous aeroelastic system of equations

$$
[M]\{\ddot{u}(t)\}+[K]\{u(t)\}-\left\{L_{a}(u(t), \dot{u}(t))\right\}=0
$$

be written into the Laplace domain. The transformation of the induced aerodynamic forces to the Laplace domain is performed by a convolution integral

$$
\left\{L_{a}(u(t), \dot{u}(t))\right\}=\int_{0}^{1} q_{\infty}\left[H\left(\frac{U_{\infty}}{b}(t-\tau)\right)\right]\{u(\tau)\} d \tau
$$

where $[H]$ represents the aerodynamic transfer function matrix. The aerodynamic loading in the Laplace domain is written as

$$
\left\{L_{a}(u(s))\right\}=q_{\infty}\left[\bar{H}\left(\frac{s b}{U_{\infty}}\right)\right]\{u(s)\}
$$

with $\left[\bar{H}\left(\frac{s b}{U_{\infty}}\right)\right]$ as the Laplace domain counterpart of $\left[H\left(\frac{U_{\infty} t}{b}\right)\right]$ and $s b / U_{\infty}$ being the non-dimensional Laplace variable. The resulting eigenvalue problem is obtained by transforming Eq. (2.13) to the Laplace domain as

$$
\left[s^{2}[M]+[K]-q_{\infty}\left[\bar{H}\left(\frac{s b}{U_{\infty}}\right)\right]\right]\{u(s)\}=0
$$

\subsubsection{Modal Approach}

Since the finite element model of aircraft structure normally contains a large amount of degrees of freedom, the size of the mass and stiffness matrices can be substantial. Directly solving the eigenvalue problem of Eq. (2.16) would be computationally 
costly [10], [16]. Transforming the structural dynamic system to modal space circumvents this problem. The resulting eigenvalues are known as the natural frequencies and the eigenvectors are the associated mode shape vectors. Therefore, in a modal representation, the structural displacements are written as a linear combination of the generalized coordinates, $\{q(t)\}$, and the physical displacements of the structure, $\{u(t)\}$, as

$$
\{u(t)\}=[\Phi]\{q(t)\}
$$

where $[\Phi]$ is the modal transformation matrix whose columns contain mode shape vectors, each associated to a given natural frequency of the structure. Normally, no more than 10 of the lowest natural modes are sufficient for a flutter analysis of a wing. The rationale of the modal approach is based on the premise that the critical flutter modes are usually due to the coupling of lower order structural modes. Thus, the structural deformation of the flutter mode can be sufficiently represented by the superposition of lower order modes [10]. The resulting aeroelastic system of equations written in the modal space is

$$
\left[s^{2}[\bar{M}]+[\bar{K}]-q_{\infty}\left[Q\left(\frac{s b}{U_{\infty}}\right)\right]\right]\{q(s)\}=0
$$

where $[\bar{M}]=[\Phi]^{\top}[M][\Phi]$ and $[\bar{K}]=[\Phi]^{\top}[K][\Phi]$ are the generalized mass and stiffness matrices, respectively, and

$$
\left[Q\left(\frac{s b}{U_{\infty}}\right)\right]=[\Phi]^{\top}\left[\bar{H}\left(\frac{s b}{U_{\infty}}\right)\right][\Phi]
$$


is the generalized aerodynamic force matrix, with $\{q(s)\}$ being the generalized coordinates vector in the Laplace domain.

\subsubsection{Simple Harmonic Motion Approach}

The computation of the aerodynamic transfer functions in the Laplace domain can be extremely difficult. For this reason, unsteady aerodynamic methods are often formulated in the frequency domain assuming simple harmonic motion. The aerodynamic transform function, in a simple harmonic reduced frequency domain, is called the AIC matrix [10].

The induced aerodynamic loading is obtained by a convolution integral which transforms the aerodynamic loading from the time domain to the Laplace domain. Since the AIC matrix is only available in the simple harmonic reduced frequency domain, the imaginary counterpart of aeroelastic system of equations, given by Eq. (2.18), is obtained with $i \omega$ replacing $s$ leading to

$$
\left[-\omega^{2}[\bar{M}]+[\bar{K}]-q_{\infty}[Q(i k)]\right]\{q(i \omega)\}=0
$$

with $\{u(i \omega)\}=[\Phi]\{q(i \omega)\}$.

\subsubsection{Interconnection of the Structure with Aerodynamics}

The location and size of the aerodynamic panels may be independent of the structural finite elements in order to allow for discretization optimization. An interpolation 
procedure is required to pass the displacements of the structural nodes to the aerodynamic control points and forces from the aerodynamic control points to the structural nodes. This procedure is based on spline function approximations [16]. A spline matrix, $[G]$, is generated using the beam spline method, the infinite plate spline method, the thin-plate spline method or the rigid-body attachment method, such that

$$
\{h(x, y, 0)\}=[G]\left\{u\left(x_{s}, y_{s}, z_{s}\right)\right\}
$$

where $\left\{u\left(x_{s}, y_{s}, z_{s}\right)\right\}$ is the finite element model nodal displacement vector and the displacement vector at the aerodynamic control points is $\{h(x, y, 0)\}$. Once the spline matrix has been generated, the force transferral from the aerodynamic control points to structural nodes can be performed accordingly

$$
\{\delta h(x, y, 0)\}^{\top}\left\{L_{a}^{a e r o}(x, y, 0)\right\}=\left\{\delta u\left(x_{s}, y_{s}, z_{s}\right)\right\}^{\top}\left\{L_{a}^{s t r}\left(x_{s}, y_{s}, z_{s}\right)\right\}
$$

where $\{\delta h(x, y, 0)\}^{\top}$ and $\left\{\delta u\left(x_{s}, y_{s}, z_{s}\right)\right\}^{\top}$ are the virtual displacements. The aerodynamic loading at the structural nodes and at the aerodynamic control points are denoted as $\left\{L_{a}^{\text {str }}\left(x_{s}, y_{s}, z_{s}\right)\right\}$ and $\left\{L_{a}^{\text {aero }}(x, y, 0)\right\}$, respectively. The relationship between the loads is given by

$$
\left\{L_{a}^{s t r}\left(x_{s}, y_{s}, z_{s}\right)\right\}=[G]^{\top}\left\{L_{a}^{a e r o}(x, y, 0)\right\}
$$

Replacing Eq. (2.10) in Eq. (2.23), using Eq. (2.21), yields

$$
\left\{L_{a}^{s t r}\left(x_{s}, y_{s}, z_{s}, i k\right)\right\}=q_{\infty}[G]^{\top}[S][A I C(i k)][F(i k)][G]\left\{u\left(x_{s}, y_{s}, z_{s}\right)\right\}
$$


The generalized aerodynamic forces matrix, in the reduced frequency domain, is given by

$$
[Q(i k)]=\left[\Phi_{a}\right]^{\top}[G]^{\top}[S][A I C(i k)][F(i k)][G]\left[\Phi_{a}\right]
$$

\subsection{Flutter Solution Techniques}

The stability of an aeroelastic system is evaluated by using flutter solution techniques. These methods are based on the solution of the eigenvalue problem with respect to a given parameter variation. Three methods of analysis are available: (1) the American (K) method; (2) a restricted but more efficient American (KE) method; and (3) the British (PK) method.

The K-method of flutter analysis considers the aerodynamic loads as complex masses. Therefore, the method becomes a vibration analysis using complex arithmetic to determine the frequencies and artificial dampings required to sustain the assumed harmonic motion. Structural damping is incorporated into the analysis as a matrix. As the K-method is a looping procedure, caution must be used if a large number of loops are specified; the analysis may take an excessive time to execute.

The KE-method is ideal when hundreds of flutter analyses are required. The KE-method is similar to the K-method; by restricting the functionality, the $\mathrm{KE}$ method becomes more efficient than the K-method. Two major restrictions are: (1) no damping matrix is allowed; and (2) no eigenvector recovery is made. The effects of structural damping are, however, included in the complex stiffness matrix. Eq. (2.20) 
becomes

$$
\left[-\omega^{2}[\bar{M}]+(1+i g)[\bar{K}]-q_{\infty}[Q(i k)]\right]\{q(i \omega)\}=0
$$

where $g$ is the added artificial damping. For both the K- and KE-methods, the term is a mathematical quantity that cannot be readily related to the physical system damping. However, these methods are capable of providing the correct prediction of the flutter boundary, which, in other words, is the flutter speed at zero damping.

The PK-method not only determines stability boundaries but provides approximate and realistic estimates of system damping at subcritical speeds that can be used to monitor flight flutter tests. The PK-method treats the aerodynamic matrices as real frequency dependent springs and dampers. This method requires less eigenvalues to solve, thereby resulting in a more efficient solver.

Although MSC/NASTRAN is capable of performing flutter analyses utilizing the K-, KE- and PK-methods, KEDLMPL can only utilize the KE-method. Thus, for consistency between the frequency domain results, the KE-method will be applied for flutter computations in the present investigations. 


\section{Chapter 3}

\section{AIC Correction Formulation}

\subsection{Overview of AIC Correction Methods}

Several procedures have been developed which relate measured or computed data to the unsteady linear aerodynamic model in order to account for nonlinear effects. The correction techniques are classified in four major classes: (1) force matching methods; (2) pressure matching methods; (3) Dau-Garner type methods; and (4) MAIC matrix replacement. Commonly employed methods for each class will be briefly discussed. Detailed information is available in Reference [16].

\subsubsection{Force Matching Methods}

The modified strip theory was the first correction technique; lift and moment coefficient derivatives obtained from Theodorsen's method were replaced by the derivatives determined from measurements. The published results were in good agreement with experimental results for the case of high aspect ratio wings only [12], [16]. 
Reference [18] developed a method to correct the subsonic discrete lifting surface theory based on a reference experimental or nonlinear computed loading. This method included a procedure to obtain a pre- or post-multiplicative operator to correct the AIC matrix by the use of weighting factors. The purpose of such operators was to match a single reference steady spanwise loading of the lifting surface. Each spanwise station corresponded to a set of panels aligned in the streamwise direction, thereby resulting in less known quantities than the necessary weighting factors. In order to circumvent this problem, the weighting factors were obtained using the least squares method and thus, there is no guarantee that the modified pressure distribution resulting from the correct AIC matrix will be identical to the experimental or nonlinear computed pressures. In the presence of shock waves, this pressure distortion can severely modify the aeroelastic behaviour in the transonic regime as the nature of the transonic dip depends on the position and strength of the shock wave. Nevertheless, the modified pressure distribution will guarantee correct sectional lift and moments [16], [18].

A method to correct a linear stripwise AIC matrix was presented by Reference [20] which related strip displacements to their loads. This procedure was based on a postmultiplication of the stripwise AIC matrix by weighting factors which were obtained from the inversion of the AIC matrix multiplied by the reference loading condition. The reference loading condition was obtained from the solution of the transonic small 
disturbance equation in a steady flow condition. The results showed agreement with experimental flutter speeds. Small differences between the results were attributed to the inaccuracy of the predicted strength and location of the shock wave using the numerical solution of the transonic small disturbance equation.

\subsubsection{Pressure Matching Methods}

Reference [22] presented a procedure based on the pre-multiplication of the AIC matrix by a diagonal weighting operator which was obtained from the ratio between the experimental and theoretical steady pressures. Similarly, Reference [23] pre-multiplied the AIC matrix by a diagonal weighting operator based on the ratio between measured and theoretical unsteady pressures related to a given downwash mode shape. Reference [24] calculated a diagonal weighting operator obtained from the ratio between the nonlinear and the linear quasi-steady pressure slopes. The correction was performed in two forms, as a multiplicative weighting operator or as an additive weighting operator. The results showed incorrect trends for computed unsteady pressure results as well as nonconservative flutter speeds [16]. The computation of the correction factors presented in References [22], [23] and [24] also produced badly conditioned corrected AIC matrices for zero or very low pressure values.

Reference [25] presented an additive correction procedure based on the increment of the linear AIC matrix by a frequency dependent nonlinear term. The nonlinear 
term was obtained from a nonlinear unsteady CFD computation of the lifting surface undergoing an impulse-type displacement. The pressures were computed from a discrete Fourier transform of the aerodynamic response in the time domain. The resulting frequency dependent unsteady pressures were then used to obtain the nonlinear term of the AIC matrix at each reduced frequency. This method was more expensive than previously discussed approaches as it required a refined time marching CFD solution. The results showed agreement with experiments for standard aeroelastic wing configuration flutter computations only [16], [25].

An extension of the correction method of Reference [18] was presented by References [26] and [27] in which pressures were used instead of loads as reference conditions. This procedure employed multiple reference pressure conditions associated with a reference mode shape. The number of the given reference steady pressures was smaller than the order of the AIC matrix. Therefore, the resulting pressure matrix, which contained the given pressures due to prescribed mode shapes, was a rectangular matrix. The computation of the weighing operator is performed by the inversion of the matrix of given pressures, requiring a minimization technique. Minimization, however, was avoided by computing of a set of linear steady pressures based on a linearly independent artificial mode shape. Since these complementary pressure conditions were known, they were included in the given reference pressure matrix. A modified square pressure matrix was then constructed in which each of its columns 
were related to a given modal displacement [16]. This pressure matrix was inverted and multiplied by a linear pressure matrix. The resulting weighting operator was a fully populated matrix. As DLM interference effects were not strongly modified, this procedure avoided severe pressure distortions. Subsonic flutter calculations were presented showing agreement with the experimental results; however, further extension into the transonic regime yielded poor results [26].

Reference [16] presented a pressure correction based on downwash weighting which preserved the mean steady flow nonlinear characteristics. The control point displacement vector was modified in order to satisfy a given reference pressure distribution. The advantage of this method was that the computed weighting factors depended on a linear system of equations instead of a simple pressure ratio. The solution of this system did not yield incorrect weighting factors even for reference conditions containing null displacements or pressures. The second advantage was that the AIC matrices resulting from the conventional modelling, based on discrete element solutions of the linearized potential flow equations (i.e. the DLM), related pressures to displacements at control points. Therefore, it was feasible to employ this pressure matching technique for the correction of the discrete element models. Reference [16] presented results using nonlinear reference conditions computed from steady state and unsteady CFD solutions. The results showed agreement with experimental flutter speeds for unsteady CFD solutions as a weighting operator was computed for all 
reduced frequencies in the aeroelastic analysis. Pressure matching procedures based on steady reference conditions failed in obtaining unsteady pressures at higher reduced frequencies due to the absence of unsteady transonic flow information in the reference conditions. The magnitude of the pressures were well approximated; however, the phases computation presented wrong trends.

\subsubsection{Dau-Garner Type Methods}

The objective of Dau-Garner correction methods was to compute approximate nonlinear unsteady pressures in the frequency domain for use in transonic flutter calculations. The pressure computation was performed with the aid of steady state semiempirical relations. The theoretical background for this class of correction methods was introduced by Reference [28] and the extension of the theory was performed by Reference [29], resulting in the Dau-Garner method.

The basic hypothesis of the Dau-Garner method was an empirical relation that assumed an equal ratio between unsteady and steady velocity potential gradients whether the flow was transonic or purely subsonic. The unsteady velocity potential gradient was expressed as a function of the given steady state velocity potential gradient. The ratio between the linear unsteady and steady velocity potential gradients was computed from a linear theory such as the DLM [16].

An additional condition was the relation between unsteady pressures and the unsteady velocity potential, which was derived from the Euler momentum equations. 
The steady velocity potential gradient was written as a function of a steady state pressure distribution, as given by the pressure to velocity potential relation derived from the Euler momentum equations. Therefore, the unsteady velocity potential distribution was written as a function of steady pressure distribution times the ratio between the unsteady and steady velocity potential gradients. The introduction of the nonlinearities associated with transonic flow was performed by the substitution of the steady state linear pressures by the nonlinear pressures. This method modified both the real and imaginary parts of the pressures with respect to a nonlinear steady state pressure distribution [16], [29], [28], [30].

The published results showed inconsistencies with the computed phase components. In most cases, the computed phases differed by approximately 50 degrees from the experimental results.

\subsubsection{Modal Aerodynamic Influence Coefficients Matrix Methods}

Body motions may be written in terms of modal generalized coordinates instead of physical displacements coordinates. The matrix operator which relates the resulting pressures modes to displacement mode shapes is called the modal aerodynamic influence coefficient matrix (MAIC). The MAIC matrix computed from linear theory is replaced by an externally computed matrix which accounts for nonlinear aerodynamic behaviour [16], [33]. 
The only MAIC method published is the Transonic Equivalent Strip (TES) method of Reference [32]. This procedure required two consecutive correction steps, a stripwise correction followed by a spanwise correction. In the first step, a chordwise mean flow correction is performed based on steady pressures obtained from CFD or experimental sources. An inverse airfoil design procedure is employed to generate a new profile of an equivalent airfoil for each of the spanwise stations of the lifting surface. The computation of the unsteady pressures follows and was based on a time domain impulse response finite difference solution of the transonic small disturbance equation. The unsteady pressures in the frequency domain are computed from the Fourier transform of the time response of these pressures. The second step consisted of a spanwise correction of the two-dimensional unsteady pressures in order to account for three-dimensional effects [16], [32], [33].

The published flutter results for cases involving isolated lifting surfaces were in good agreement with the experimental data. The TES method is, however, unable to efficiently handle complex typical section profiles such as a combination of wingpylon-nacelles or external stores with the wing profile [16].

\subsection{Derivation of Selected Correction Method}

The selected correction procedure for the present work was the unsteady pressure matching method of Reference [16]. This method, based on a downwash weighting approach, has the following advantages: (1) the nonlinear flow characteristics in the 
transonic regime are preserved; (2) reference conditions can be based on unsteady CFD or experimental data; (3) reference conditions containing null displacements or pressures do not yield incorrect weighting factors; and (4) ease of implementation into the DLM.

The following section will detail the AIC correction procedure. Refer to Reference [16] for the detailed derivation of the steady and unsteady correction procedure.

\subsubsection{Theoretical Derivation}

The basic equation over which the procedure is developed is the algebraic pressure to downwash relationship that is derived from the application of discrete element kernel function methods to model the linearized potential flow equation [16]. This relation is rewritten in a simplified form as a function only of the reduced frequency as

$$
\left\{\Delta C_{p}(i k)\right\}=[A I C(i k)]\{\mathrm{w}(i k)\}
$$

The reference conditions are based on frequency-dependent pressures computed from the time domain aerodynamic response. Pressure time histories result from lifting surface displacements due to a prescribed motion. These displacements are arbitrary and may be an impulse-type or an oscillating harmonic motion [16]. For the present work, the unsteady pressure differences computation was performed based on a time domain CFD solution of the Euler and Navier-Stokes equations, using the finite volume approach presented in Chapter 4. 
The chosen displacement may be any unsteady motion associated with a displacement mode shape of the lifting surface. In the present work, the motion selected was a rigid body harmonic pitching rotation around the root midchord axis of the lifting surface at a reduced frequency, $k$. This motion leads to an unsteady downwash, regarded as an unsteady perturbation in angle of attack with the amplitude equal to the amplitude of the pitching motion, $\Delta \alpha[16]$.

Nonlinear pressure coefficient differences, denoted by the superscript $n l$, are computed from the solutions of the Navier-Stokes or Euler equations as

$$
\left\{\Delta C_{p}^{n l}\right\}=\left\{C_{p}^{l}\right\}-\left\{C_{p}^{u}\right\}
$$

where the superscripts $l$ and $u$ denote the lower and upper surfaces of the lifting surface, respectively.

As the linear aerodynamic model is defined in the frequency domain, the reference time domain pressure differences must be transformed into the frequency domain. The following Fourier transformations were employed in order to obtain the first harmonic frequency domain components of the pressure differences

$$
\begin{aligned}
& \operatorname{Re}\left(\Delta C_{p}^{n l}(i k)\right)=\frac{k}{\pi \Delta \alpha} \int_{\tau_{1}}^{\tau_{1}+\pi / k} \Delta C_{p}(\tau) \sin (k \tau) \mathrm{d} \tau \\
& \operatorname{Im}\left(\Delta C_{p}^{n l}(i k)\right)=\frac{k}{\pi \Delta \alpha} \int_{\tau_{1}}^{\tau_{1}+\pi / k} \Delta C_{p}(\tau) \cos (k \tau) \mathrm{d} \tau
\end{aligned}
$$

where $\tau$ is the non-dimensional time, as defined in the non-dimensionalization of the Euler and Navier-Stokes equations; refer to Chapter 5 for the validation of the 
computational procedure for unsteady flow computations.

The complex first harmonic components of the pressure coefficient differences are obtained from the real and imaginary part of the first harmonic components, given by Eqs. (3.3) and (3.4) as

$$
\left\{\Delta C_{p}^{n l}\right\}=\left(\operatorname{Re}\left\{\Delta C_{p}^{n l}(i k)\right\}+\operatorname{Im}\left\{\Delta C_{p}^{n l}(i k)\right\}\right)
$$

Eq. (3.1) is rewritten as a function of a frequency dependant weighing operator, $W T$, given by

$$
\left\{\Delta C_{p}^{n l}\right\}=[A I C(i k)][W T(i k)]\{\overline{\mathrm{w}}(i k)\}
$$

where $\{\overline{\mathrm{w}}(i k)\}$ is the downwash vector divided by the amplitude of the pitching motion, $\Delta \alpha$. The diagonal weighing matrix coefficients are obtained from the ratio between a modified unsteady downwash, computed by

$$
\left\{\mathrm{w}^{n l}(i k)\right\}=[A I C(i k)]^{-1}\left\{\Delta C_{p}^{n l}\right\}
$$

and the known downwash associated with the prescribed lifting surface motion, leading to $[16]$

$$
\left(W T(i k)_{i i}\right)=\frac{\left(\mathrm{w}^{n l}(i k)\right)_{i}}{(\overline{\mathrm{w}}(i k))_{i}}
$$

Therefore, the nonlinear unsteady aerodynamic loading is performed by incorporating the correcting weighting operator into Eq. (2.10), leading to an approximate aerodynamic loading given by [16]

$$
\left\{L_{a}^{n l}(i k)\right\}=q_{\infty}[S][A I C(i k)][W T(i k)]\{\mathrm{w}(i k)\}
$$




\section{Chapter 4}

\section{Time Marching Formulation}

\subsection{Numerical Fluid Methods}

The time marching simulations will be performed using the PMB (Parallel MultiBlock) code developed at the University of Glasgow. This implicit code features a finite-volume Euler and RANS CFD solver with a proven capability of accurately capturing transonic effects. A brief derivation of the Euler and RANS solvers follows. For detailed derivations, refer to References [4], [40], [43] and [44].

\subsubsection{Euler Equations}

The three-dimensional Euler equations can be written in non-dimensional conservative form as

$$
\frac{\partial \mathbf{W}}{\partial t}+\frac{\partial \mathbf{F}}{\partial x}+\frac{\partial \mathbf{G}}{\partial y}+\frac{\partial \mathbf{H}}{\partial z}=0
$$


where the vector $\mathbf{W}=(\rho, \rho u, \rho v, \rho w, \rho E)^{\top}$ denotes the vector of conservative variables. The flux vectors, $\mathbf{F}, \mathbf{G}$ and $\mathbf{H}$, are

$$
\begin{aligned}
\mathbf{F} & =\left(\begin{array}{c}
\rho U \\
\rho u U+p \\
\rho v U \\
\rho w U \\
U(\rho E+p)+x_{t} p
\end{array}\right) \\
\mathbf{G} & =\left(\begin{array}{c}
\rho V \\
\rho u V \\
\rho v V+p \\
\rho w V \\
V(\rho E+p)+y_{t} p
\end{array}\right) \\
\mathbf{H} & =\left(\begin{array}{c}
\rho W \\
\rho u W \\
\rho v W \\
\rho w W+p \\
W(\rho E+p)+z_{t} p
\end{array}\right)
\end{aligned}
$$

where $\rho, u, v, w, p$ and $E$ denote the density, the three Cartesian components of the velocity, the pressure and the specific total energy, respectively. The terms $U, V$ and $W$ are the contravariant velocities defined by

$$
\begin{aligned}
U & =u-x_{t} \\
V & =v-y_{t} \\
W & =w-z_{t}
\end{aligned}
$$

where $x_{t}, y_{t}$ and $z_{t}$ are the grid speeds in the Cartesian directions. 


\subsubsection{Navier-Stokes Equations}

The three-dimensional Reynolds Averaged Navier-Stokes (RANS) equations can be written in non-dimensional conservative form as

$$
\frac{\partial \mathbf{W}}{\partial t}+\frac{\partial \mathbf{F}^{i}-\mathbf{F}^{v}}{\partial x}+\frac{\partial \mathbf{G}^{i}-\mathbf{G}^{v}}{\partial y}+\frac{\partial \mathbf{H}^{i}-\mathbf{H}^{v}}{\partial z}=0
$$

The conservative form of the Navier-Stokes equations are obtained through a derivation based on a spatially fixed control volume. The flux vectors $\mathbf{F}, \mathbf{G}$ and $\mathbf{H}$ are composed of inviscid terms as described with the superscript $i$ and diffusive viscous terms as described by the superscript $v$ [45]. The inviscid flux terms are

$$
\begin{gathered}
\mathbf{F}^{i}=\left(\begin{array}{c}
\rho U \\
\rho u U+\xi_{x} p \\
\rho v U+\xi_{y} p \\
\rho w U+\xi_{z} p \\
\rho U H
\end{array}\right) \\
\mathbf{G}^{i}=\left(\begin{array}{c}
\rho \\
\rho V \\
\rho v V+\eta_{y} p \\
\rho w V+\eta_{z} p \\
\rho V H \\
\rho W \\
\rho u W+\varsigma_{x} p \\
\rho v W+\varsigma_{y} p \\
\rho w W+\varsigma_{z} p \\
\rho W H
\end{array}\right)
\end{gathered}
$$

where $H$ denotes enthalpy. The terms $U, V$ and $W$ are defined in the local curvilinear 
$\xi, \eta, \varsigma$ coordinate system as

$$
\begin{aligned}
U & =\xi_{x} u+\xi_{y} v+\xi_{z} w \\
V & =\eta_{x} u+\eta_{y} v+\eta_{z} w \\
W & =\varsigma_{x} u+\varsigma_{y} v+\varsigma_{z} w
\end{aligned}
$$

The viscous terms are

$$
\begin{aligned}
& \mathbf{F}^{v}=\frac{1}{\operatorname{Re}}\left(\begin{array}{c}
0 \\
\tau_{x x} \\
\tau_{x y} \\
\tau_{x z} \\
u \tau_{x x}+v \tau_{x y}+w \tau_{x z}+q_{x}
\end{array}\right) \\
& \mathbf{G}^{v}=\frac{1}{\operatorname{Re}}\left(\begin{array}{c}
0 \\
\tau_{x y} \\
\tau_{y y} \\
\tau_{y z} \\
u \tau_{x y}+v \tau_{y y}+w \tau_{y z}+q_{y}
\end{array}\right) \\
& \mathbf{H}^{v}=\frac{1}{\operatorname{Re}}\left(\begin{array}{c}
0 \\
\tau_{x z} \\
\tau_{y z} \\
\tau_{z z} \\
u \tau_{z x}+v \tau_{z y}+w \tau_{z z}+q_{z}
\end{array}\right)
\end{aligned}
$$


where Re denotes the Reynolds number. The stress tensors are defined as

$$
\begin{aligned}
\tau_{x x} & =-\left(\mu+\mu_{T}\right)\left[2 \frac{\partial u}{\partial x}-\frac{2}{3}\left(\frac{\partial u}{\partial x}+\frac{\partial v}{\partial y}+\frac{\partial w}{\partial z}\right)\right]+\frac{2}{3} \rho k \\
\tau_{y y} & =-\left(\mu+\mu_{T}\right)\left[2 \frac{\partial v}{\partial y}-\frac{2}{3}\left(\frac{\partial u}{\partial x}+\frac{\partial v}{\partial y}+\frac{\partial w}{\partial z}\right)\right]+\frac{2}{3} \rho k \\
\tau_{z z} & =-\left(\mu+\mu_{T}\right)\left[2 \frac{\partial w}{\partial z}-\frac{2}{3}\left(\frac{\partial u}{\partial x}+\frac{\partial v}{\partial y}+\frac{\partial w}{\partial z}\right)\right]+\frac{2}{3} \rho k \\
\tau_{x y} & =-\left(\mu+\mu_{T}\right)\left[\frac{\partial u}{\partial y}+\frac{\partial v}{\partial x}\right] \\
\tau_{x z} & =-\left(\mu+\mu_{T}\right)\left[\frac{\partial u}{\partial z}+\frac{\partial w}{\partial x}\right] \\
\tau_{y z} & =-\left(\mu+\mu_{T}\right)\left[\frac{\partial v}{\partial z}+\frac{\partial w}{\partial y}\right]
\end{aligned}
$$

where $k, \mu_{T}$ and $P r_{T}$ are the turbulent kinetic energy, the eddy viscosity and the turbulent Prandtl number, respectively. The heat flux vectors are defined as

$$
\begin{aligned}
& q_{x}=-\frac{1}{(\gamma-1) M_{\infty}^{2}}\left[\frac{\mu}{P r}+\frac{\mu_{T}}{P r_{T}}\right] \frac{\partial T}{\partial x} \\
& q_{y}=-\frac{1}{(\gamma-1) M_{\infty}^{2}}\left[\frac{\mu}{P r}+\frac{\mu_{T}}{P r_{T}}\right] \frac{\partial T}{\partial y} \\
& q_{z}=-\frac{1}{(\gamma-1) M_{\infty}^{2}}\left[\frac{\mu}{P r}+\frac{\mu_{T}}{P r_{T}}\right] \frac{\partial T}{\partial z}
\end{aligned}
$$

where $T$ denotes the static temperature.

The Navier-Stokes equations permit the complete and accurate solution of a physical flow. In order to allow this solution to occur in a timely manner, turbulence modelling is introduced as a means of predicting turbulent transition, flow separation and flow reattachment. The RANS equation decomposes the flow variables into a time averaged component and a turbulent fluctuation describing the instantaneous variation from the mean value [43]. 
The turbulence properties of the flow are modelled by the turbulent viscosity, turbulent kinetic energy and an additional set of closure equations. The closure equations, known as turbulence models, present a mathematical resolution for the effects of turbulence. Refer to References [43] and [45] for detailed derivations.

\subsubsection{Steady State Solver}

The Euler and Navier-Stokes equations are discretized onto curvilinear multi-block body conforming grids using a cell-centred finite volume method. The partial differential equations of Eqs. (4.1) and (4.4) are converted into a set of ordinary differential equations which can be written as

$$
\frac{\mathrm{d}}{\mathrm{d} t}\left(V_{i, j, k} \mathbf{W}_{i, j, k}\right)=-\mathbf{R}_{i, j, k}(\mathbf{W})
$$

where $\mathbf{W}$ and $\mathbf{R}$ are the vectors of the cell conserved variables and the cell residuals, respectively. The volume of the cell is denoted by $V_{i, j, k}$. The convective terms are discretized using Osher's upwind method. MUSCL variable extrapolation is used to provide second-order accuracy with the Van Albada limiter to prevent spurious oscillations around shock waves [44]. Two layers of ghost cells are generated outside of the computational domain providing boundary conditions. For the far-field, the ghost cells are set equal to the freestream conditions. For viscous flows, the no-slip boundary condition is imposed at the wall. For inviscid flows, the normal component of velocity is set equal to zero [45]. 
A numerical integration allows the solution of the set of differential equations created by the finite volume method. The steady state solution is determined from an implicit time-marching scheme according to

$$
\frac{\mathbf{W}_{i, j, k}^{n+1}-\mathbf{W}_{i, j, k}^{n}}{\Delta t}=-\frac{1}{V_{i, j, k}} \mathbf{R}_{i, j, k}\left(\mathbf{W}_{i, j, k}^{n+1}\right)
$$

where the superscripts $n$ and $n+1$ refer to time $n \Delta t$ and $(n+1) \Delta t$, respectively. The numerical integration expressed above represents a system of non-linear equations. In order to simplify the solution procedure, the residual term is linearized

$$
\begin{aligned}
\mathbf{R}_{i, j, k}\left(\mathbf{W}^{n+1}\right) & =\mathbf{R}_{i, j, k}\left(\mathbf{W}^{n}\right)+\frac{\partial \mathbf{R}_{i, j, k}}{\partial t} \Delta t+O\left(\Delta t^{2}\right) \\
& \approx \mathbf{R}_{i, j, k}^{n}\left(\mathbf{W}^{n}\right)+\frac{\partial \mathbf{R}_{i, j, k}}{\partial \mathbf{W}_{i, j, k}} \frac{\partial \mathbf{W}_{i, j, k}}{\partial t} \Delta t \\
& \approx \mathbf{R}_{i, j, k}^{n}\left(\mathbf{W}^{n}\right)+\frac{\partial \mathbf{R}_{i, j, k}}{\partial \mathbf{W}_{i, j, k}} \Delta \mathbf{W}_{i, j, k}
\end{aligned}
$$

where $\Delta \mathbf{W}_{i, j, k}=\mathbf{W}_{i, j, k}^{n+1}-\mathbf{W}_{i, j, k}^{n}$, and the integration becomes linear according to

$$
\left[\frac{V_{i, j, k}}{\Delta t} \mathbf{I}+\frac{\partial \mathbf{R}_{i, j, k}}{\partial \mathbf{W}_{i, j, k}}\right]=-\mathbf{R}_{i, j, k}^{n}\left(\mathbf{W}^{n}\right)
$$

The above linear system is solved through a Generalized Conjugate Gradient (GCG) method. The GCG method provides an approximate solution of a linear system by minimizing an error function in a finite-dimensional space of potential solution vectors. The preconditioning strategy for the linear system is based on a block incomplete lower-upper (BILU) factorization. This was selected because the sparsity pattern of the BILU method is the same as that of the Jacobian matrix. The BILU 
factorization is decoupled between blocks and thus, does not restrict the efficiency of parallel computation [45].

Since implicit schemes require special treatment prior to the full fledged resolution of a computational domain, the flow is initially solved explicitly. This allows for some smoothing of the flow field before the solver can be switched to the less robust but much faster implicit scheme. The resulting Jacobian matrix has more than one nonzero entries per row [43].

The solution for the steady state turbulent case is identical to the steady state mean flow case described above. The eddy viscosity is determined from the turbulent kinetic energy, and other pertinent values, and then used to calculate the mean flow values, which are in turn used to update the turbulent terms. An approximate Jacobian matrix is used as the source term by disregarding the production terms and accounting only for the dissipation terms [45].

\subsubsection{Unsteady Solver}

The unsteady solver enables time-accurate simulations of unsteady flows on meshes. The case described here is for turbulent flow and accounts for mesh deformation. The laminar and inviscid cases are calculable through the elimination of terms and constant mesh size is solvable without adjustment [45].

The basis of the unsteady solver is the multigrid false-time formulation developed by Jameson. The Jameson formulation for the updated steady state problem is given 
by

$$
\begin{gathered}
\mathbf{R}_{i, j, k}^{*}=\frac{3 \mathbf{w}_{i, j, k}^{n+1} V_{i, j, k}^{n+1}-4 \mathbf{w}_{i, j, k}^{n} V_{i, j, k}^{n}+\mathbf{w}_{i, j, k}^{n-1} V_{i, j, k}^{n-1}}{2 \Delta t}+\mathbf{R}_{i, j, k}\left(\tilde{\mathbf{w}}_{i, j, k}^{k_{m}}, \tilde{\mathbf{q}}_{i, j, k}^{k_{t}}\right)=0 \\
\mathbf{Q}_{i, j, k}^{*}=\frac{3 \mathbf{q}_{i, j, k}^{n+1} V_{i, j, k}^{n+1}-4 \mathbf{q}_{i, j, k}^{n} V_{i, j, k}^{n}+\mathbf{q}_{i, j, k}^{n-1} V_{i, j, k}^{n-1}}{2 \Delta t}+\mathbf{Q}_{i, j, k}\left(\tilde{\mathbf{w}}_{i, j, k}^{l_{m}}, \tilde{\mathbf{q}}_{i, j, k}^{l_{t}}\right)=0
\end{gathered}
$$

where the superscripts $k_{m}, k_{t}, l_{t}$ and $l_{t}$ represent the time level of the spatially descritized variables. In this formulation, the only modification to the laminar case is the addition of the eddy viscosity from the previous time step. Since the turbulence model is tied only to the eddy viscosity, it allows a two equation turbulence model to be implemented without modification to the mean flow solver and is the method of choice for the computational code [45].

In order to solve Jameson's equations for the updated steady state problem, a false or pseudo time, $\tau$, is introduced, yielding

$$
\begin{aligned}
& \frac{\mathbf{w}_{i, j, k}^{n+1, m+1} V_{i, j, k}^{n+\mathbf{1}, m+1}-\mathbf{w}_{i, j, k}^{n+1, m} V_{i, j, k}^{n+1, m}}{\Delta \tau}+ \\
& \frac{\mathbf{w}_{i, j, k}^{k_{m}} V_{i, j, k}^{k_{m}}-4 \mathbf{w}_{i, j, k}^{n} V_{i, j, k}^{n}+\mathbf{w}_{i, j, k}^{n-1} V_{i, j, k}^{n-1}}{2 \Delta t}+\mathbf{R}_{i, j, k}\left(\tilde{\mathbf{w}}_{i, j, k}^{k_{m}}, \mathbf{q}_{i, j, k}^{k_{t}}\right)=0
\end{aligned}
$$

where the superscript $n$ continues to describe the real time step and the superscript $m$ is introduced in order to describe the pseudo time step. An implicit time stepping method for pseudo time is employed in PMB, with $k_{m}=l_{m}=l_{t}=n-1, m-1$ and $k_{t}=n-1, m$. The solution of the equations is decoupled by freezing values and the real time stepping proceeds without sequential error thereby providing second order accuracy in time [45]. 


\subsubsection{Turbulence Model}

Closure equations in the form of a turbulence model must be implemented in order to have a closed solution to the RANS equations. The Shear Stress Transport (SST) turbulence model was selected as it is applicable to cases involving complex surface interactions [45].

The SST model of Reference [46] blends the $k-\epsilon$ model (typically accepted for turbulence modelling outside of the boundary layer) and the $k-\omega$ model (accepted for turbulence modelling within the boundary layer). A two equation turbulence model can be introduced through the eddy viscosity term [45]. The eddy viscosity term becomes

$$
\mu_{T}=\frac{\rho k / \omega}{\max \left[1 ; \Omega F_{2} /\left(a_{1} \omega\right)\right]}, a_{1}=0.31
$$

which describes that in a turbulent boundary layer the maximum value of eddy viscosity is limited by forcing the shear stress to be bound by the turbulent kinetic energy $\omega$ times a constant $a_{1}$. Additionally, the absolute vorticity is multiplied by a forcing function $F_{2}$ which is defined as a function of the wall distance $y$ according to

$$
F_{2}=\tanh \left[\left(\max \left[2 \frac{\sqrt{k}}{0.09 \omega y} ; \frac{500 \mu}{\rho y^{2} \omega}\right]\right)^{2}\right]
$$

The two equations of the SST model are defined in terms of the blending function $F_{1}$, which established the appropriate mix of the $\epsilon$ and $\omega$ model equations [45]. The 
transport equation is given by

$$
\rho \frac{\partial k}{\partial t}+\rho V \nabla k-\frac{1}{\operatorname{Re}} \nabla\left[\left(\mu+\sigma^{*} \mu_{T}\right) \nabla k\right]=P_{k}-\beta^{*} \rho k \omega
$$

and the specific dissipation rate by

$$
\rho \frac{\partial \omega}{\partial t}+\rho V \nabla \omega-\frac{1}{\operatorname{Re}} \nabla\left[\left(\mu+\sigma_{\omega} \mu_{T}\right) \nabla \omega\right]=P_{\omega}-\beta \rho \omega^{2}+2\left(1-F_{1}\right) \frac{\rho \sigma_{\omega 2}}{\omega} \nabla k \nabla \omega
$$

The blending function $F_{1}$ is set to unity for a no-slip boundary and goes to zero towards the outer edge of the boundary layer. The function is defined as

$$
F_{1}=\tanh \left[\left[\min \left(\max \left[\frac{\sqrt{k}}{0.09 \omega y} ; \frac{500 \mu}{\rho y^{2} \omega}\right] ; \frac{4 \rho \sigma_{\omega 2} k}{C D_{k \omega} y^{2}}\right)\right]^{4}\right]
$$

where $C D_{k \omega}$ is the cross-diffusion term for the $k-\omega$ turbulence model and is defined as

$$
C D_{k \omega}=\max \left[\frac{2 \rho \sigma_{\omega 2}}{\omega} \nabla k \nabla \omega ; 10^{-20}\right]
$$

The appropriate constants must also be determined from the $k-\epsilon$ and $k-\omega$ models. Three coefficients remain constant for all cases:

$$
a_{1}=0.31, \quad \beta^{*}=0.09, \quad \kappa=0.41
$$

The remainder of the coefficients, $\beta, \gamma, \sigma_{k}$ and $\sigma_{\omega}$ are found by blending the constants from the other two turbulence models. Allowing $\phi$ to represent a characteristic coefficient with $\phi_{1}$ representing the value of the coefficients in the $k-\omega$ model and $\phi_{2}$ for the $k-\epsilon$ model, the pattern of coefficient blending according to $F_{1}$ is [45]

$$
\phi=F_{1} \phi_{1}+\left(1-F_{1}\right) \phi_{2}
$$


The coefficients of the $k-\omega$ model are

$$
\sigma_{k 1}=0.85, \sigma_{w 1}=0.85, \quad \beta_{1}=0.075, \gamma_{1}=\beta_{1} / \beta^{*}-\sigma_{\omega 1} \kappa^{2} / \sqrt{\beta^{*}}=0.553
$$

and for the $k-\epsilon$ model are

$$
\sigma_{k 2}=1.0, \quad \sigma_{w 2}=0.856, \quad \beta_{2}=0.0828, \quad \gamma_{2}=\beta_{2} / \beta^{*}-\sigma_{\omega 2} \kappa^{2} / \sqrt{\beta^{*}}=0.440
$$

\subsection{Structural Solver}

As the geometries of interest deform during the prescribed motion, the aerodynamic mesh must deform rather than rigidly translate and rotate. This is achieved for both flow models using Transfinite Interpolation of Displacements (TFI) within the blocks containing the wing [44]. The wing surface deflections are interpolated to the volume grid points $x_{i j k}$ as

$$
\delta x_{i j k}=\varphi_{j}^{0} x_{a, i k}
$$

where $\varphi_{j}^{0}$ are values of a blending function which varies between one at the wing surface $(j=1)$ and zero at the block face opposite. The surface deflections $x_{a, i k}$ are obtained from the transformation of the deflections on the structural grid. The grid speeds can be obtained by differentiating Eq. (4.27).

Finite element methods allow for the static and dynamic response of a structure to be determined. Mass $[M]$ and stiffness $[K]$ matrices are used to determine the equation of motion of an elastic structure subjected to an external force $\left\{f_{s}\right\}$

$$
[M]\{\ddot{u}\}+[K]\{u\}=\left\{f_{s}\right\}
$$


where $\{u\}$ denotes the vector of structural displacements. Note, the linear structural system is determined prior to performing flutter calculations. In the present work, the commercial package MSC/NASTRAN was employed to determine the $[M]$ and $[K]$ matrices.

The structural displacements of the structure are written as a linear combination of the generalized coordinates, $\{q\}$, and the physical displacements of the structure as

$$
\{u\}=[\Phi]\{q\}
$$

where $[\Phi]$ is the modal transformation matrix. The eigenvalues for this system are solved and scaled so that

$$
[\Phi]^{\top}[M][\Phi]=1
$$

Projecting the finite element equations onto the mode shapes results in

$$
\frac{\mathrm{d}^{2}\{q\}}{\mathrm{d} t^{2}}+\omega^{2}\{q\}=[\Phi]^{\top}\left\{f_{s}\right\}
$$

This equation can be solved by a two stage Runge-Kutta method, which requires a knowledge of $f_{s}^{n}$ and $f_{s}^{n+1}$. To avoid introducing sequencing errors by approximating the term $f_{s}^{n+1}$, the Runge-Kutta solution is iterated in pseudo time along with the fluid solution. At convergence of the pseudo time iterations, the structural and fluid solutions are properly sequenced in time [44].

Achieving effective information transfer between the structural and fluid grids is complex; the coupled solver needs to accurately determine the displacement of the 
fluid grid points based on the deflections of the structural grid points. The latter are caused by the fluid forces on the structural grid points which must be accurately determined from the known forces on the fluid surface grid points. The two grids are not, in general, coincident and where CFD is used to derive the aerodynamic forces it is unlikely that the grids will have common interfaces. The Constant Volume Tetrahedron (CVT) scheme was proposed to provide a consistent and accurate transformation procedure for coupling fluid and structure under these circumstances [44]. Refer to Reference [4] for the detailed CVT derivation. 


\section{Chapter 5}

\section{Test Cases}

\subsection{Validation of the Computational Procedure}

The finite difference solutions of the Euler and Navier-Stokes equations were applied to the simulation of the F-5 wing. This wing was selected as significant three-dimensional effects occur due to the low aspect ratio. The F-5 wing is a fighter type wing with a high leading edge sweep and a thickness of $5 \%$ of chord. Refer to References [47] and [48] for the description of the wing geometry, test conditions and the experimental results. Note in Chapter 5.2, the AGARD 445.6 wing was selected as the test case for flutter; however, experimental pressure data was not measured during the flutter tests. Therefore, the F-5 wing was employed to validate the finite difference solutions of the Euler and Navier-Stokes equations and the AGARD 445.6 wing was employed to validate the time and frequency domain flutter solutions. 


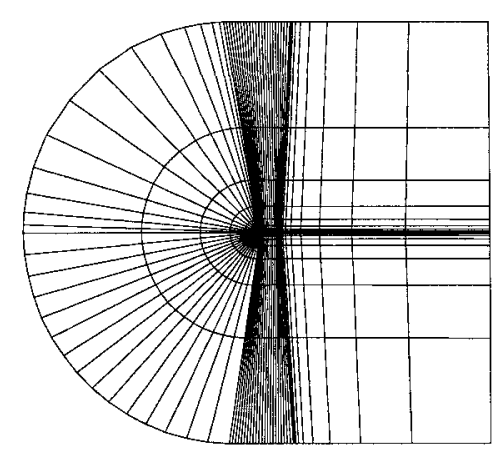

(a) Root Section

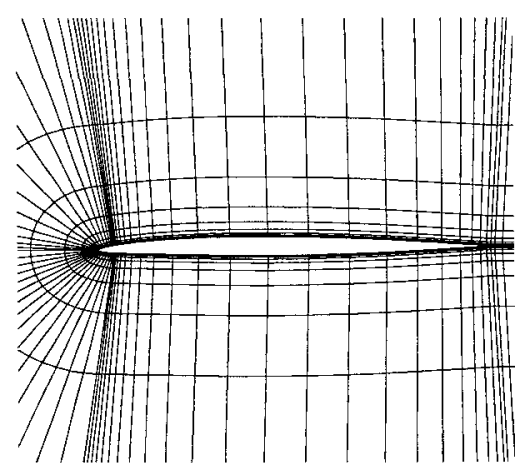

(b) Zoom of Root Section

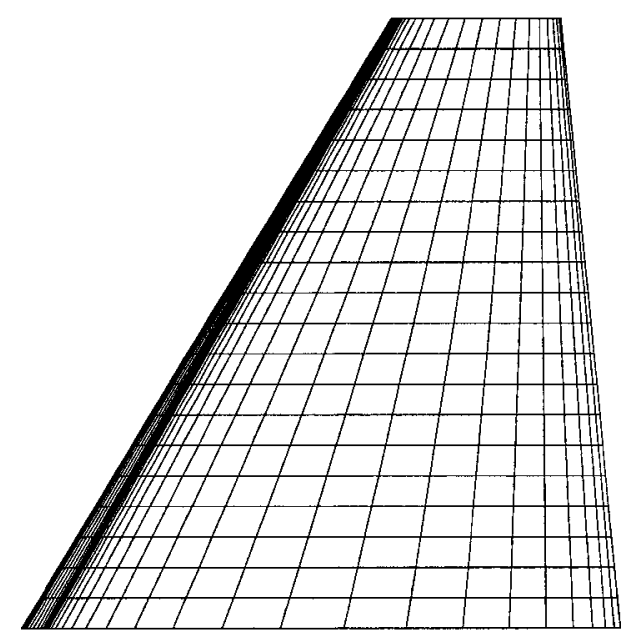

(c) Upper Wing Surface

Figure 5.1: F-5 wing fluid mesh.

\subsubsection{CFD Fluid Meshes}

Structured, multiblocked C-H medium and coarse grids were generated by a proprietary in-house code for use in both the Euler and Navier-Stokes simulations. Refer to Fig. 5.1. The medium grid contained 613,972 nodes with 7,730 points on the wing surface. The coarse grid contained 85,274 nodes with 2,374 points on the wing surface.

A grid convergence study was conducted for a steady case at zero angle of attack, 

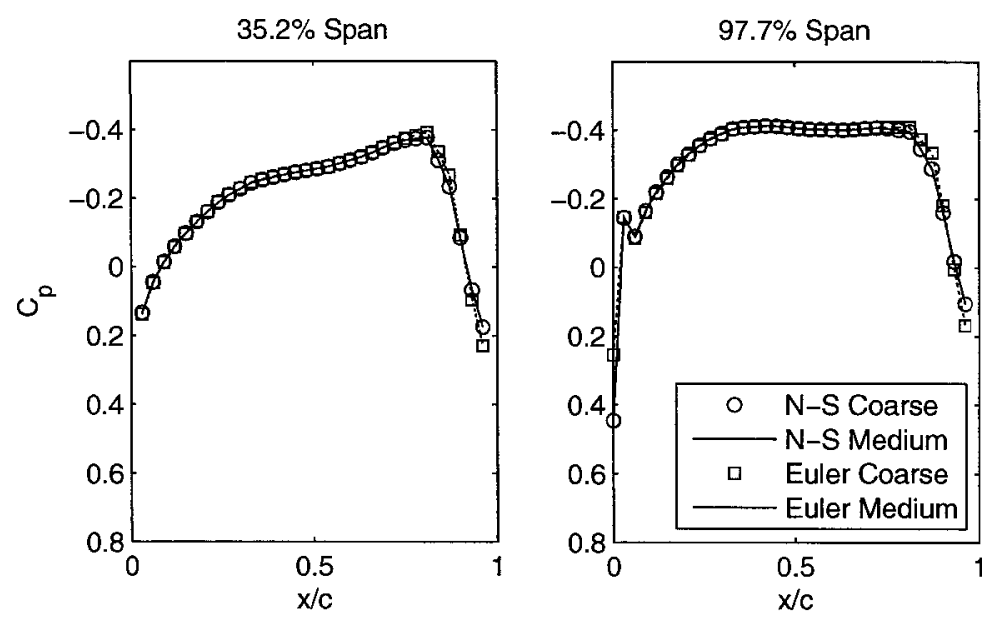

Figure 5.2: Steady $C_{p}$ over F-5 upper wing surface at $M_{\infty}=0.95$.

Mach number of 0.95 and a Reynolds number of $11 \times 10^{6}$ based on mean aerodynamic chord. The results for the Euler and Navier-Stokes solutions are shown in Fig. 5.2 for two wing stations. Both the medium and coarse grids show good agreement for the Euler and Navier-Stokes solutions. Therefore, the coarse F-5 wing grid was employed to validate the computational procedure.

\subsubsection{Steady Flow}

Steady state simulations of the F-5 wing were performed for validation of the Euler and Navier-Stokes numerical methods. The undisturbed flow conditions are shown in Table 5.1. Transonic effects are high for these flow conditions due to the presence of a strong shock wave. Note, the Reynolds number is scaled by the wing root chord $c_{r}=0.6396 \mathrm{~m}$

The steady pressures and experimental measurements are presented in Fig. 5.3 
Table 5.1: Steady flow conditions for F-5 wing.

\begin{tabular}{cc}
\hline \hline Description & Value \\
\hline Mach Number & 0.95 \\
Reynolds Number & $11 \times 10^{6}$ \\
Angle of Attack & $0.0^{\circ}$ \\
\hline \hline
\end{tabular}

for three spanwise stations. In order to evaluate the numerical method for use in the AIC correction procedure, the steady lifting pressures, $\Delta C_{p}$, are also shown. Good agreement is obtained for both upper and lower surfaces. The Euler solution required $65 \%$ less computational time to complete in comparision to the Navier-Stokes solution, yet produced virtually identical results. Refer to Fig. 5.4 for the convergence history.

\subsubsection{Unsteady Flow}

The time domain unsteady flow simulations were performed from a steady state converged solution. The unsteady flow simulation initiates a rigid body harmonic pitching rotation about the root midchord axis of the wing at a reduced frequency, $k$. The simulation parameters are shown in Table 5.2. Refer to Chapter 3.2.1 for the Fourier transformations that were applied to the pressure time histories.

Table 5.2: Unsteady flow conditions for F-5 wing.

\begin{tabular}{cc}
\hline \hline Description & Value \\
\hline Mach Number & 0.95 \\
Reynolds Number & $11 \times 10^{6}$ \\
Reduced Frequency & 0.264 \\
Pitching Motion & $\alpha(t)=0.0^{\circ}+0.222^{\circ} \sin (2 k t)$ \\
Number of Time Steps per Cycle & 20 \\
\hline \hline
\end{tabular}



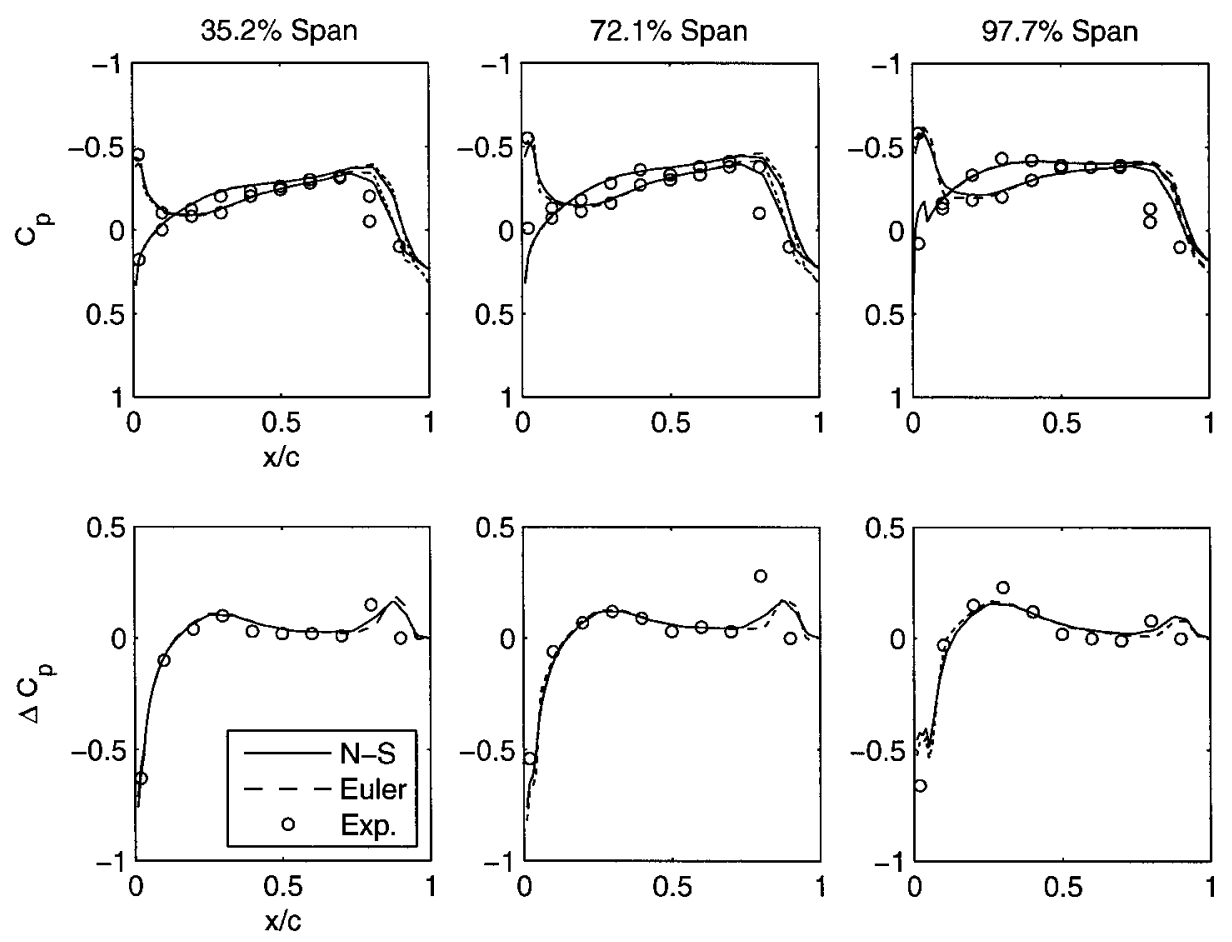

Figure 5.3: Steady $C_{p}$ and $\Delta C_{p}$ over F-5 wing at $M_{\infty}=0.95$.

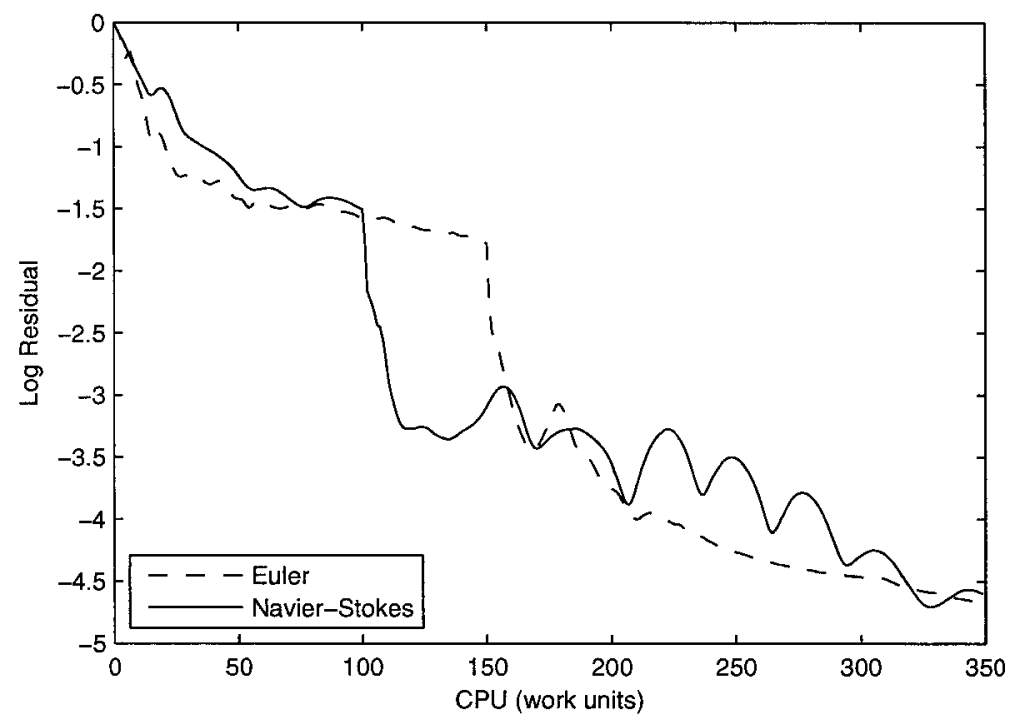

Figure 5.4: Steady state convergence for F-5 wing at $M_{\infty}=0.95$. 
The simulation was run for two and three complete cycles in order to avoid undesired transient effects [16]. Refer to Fig. 5.5. Two cycles are adequate to avoid these effects for both the Euler and the Navier-Stokes solutions. Therefore, for the present investigation, unsteady pressures are acquired from the second cycle.

Computed unsteady pressures and experimental measurements are presented in Fig. 5.6 for three spanwise stations. The unsteady lifting pressures are presented in Fig. 5.7. Good agreement of the unsteady pressures was observed. The strength and location of the primary shock wave, however, varied slightly; the Euler solution overestimated the strength whereas the Navier-Stokes solution underestimated the strength.

\subsection{Flutter Test Case}

The AGARD 445.6 wing was selected as the test case since flutter measurements are available for a wide range of Mach numbers. Results have also been published in various papers on computational aeroelasticity, including References [4], [49] and [50]. The wing has a quarter chord sweep of $45^{\circ}$, an aspect ratio of 1.65 , a taper ratio of 0.66 and a constant NACA 65A004 symmetric profile. Wind tunnel testing was conducted in the NASA Langley Transonic Dynamics Tunnel and the results were published in 1963. Various wing models were tested; the case most commonly used in computational aeroelasticity papers is the weakened wing model at zero angle of attack in air. This model was constructed of laminated mahogany and had holes 

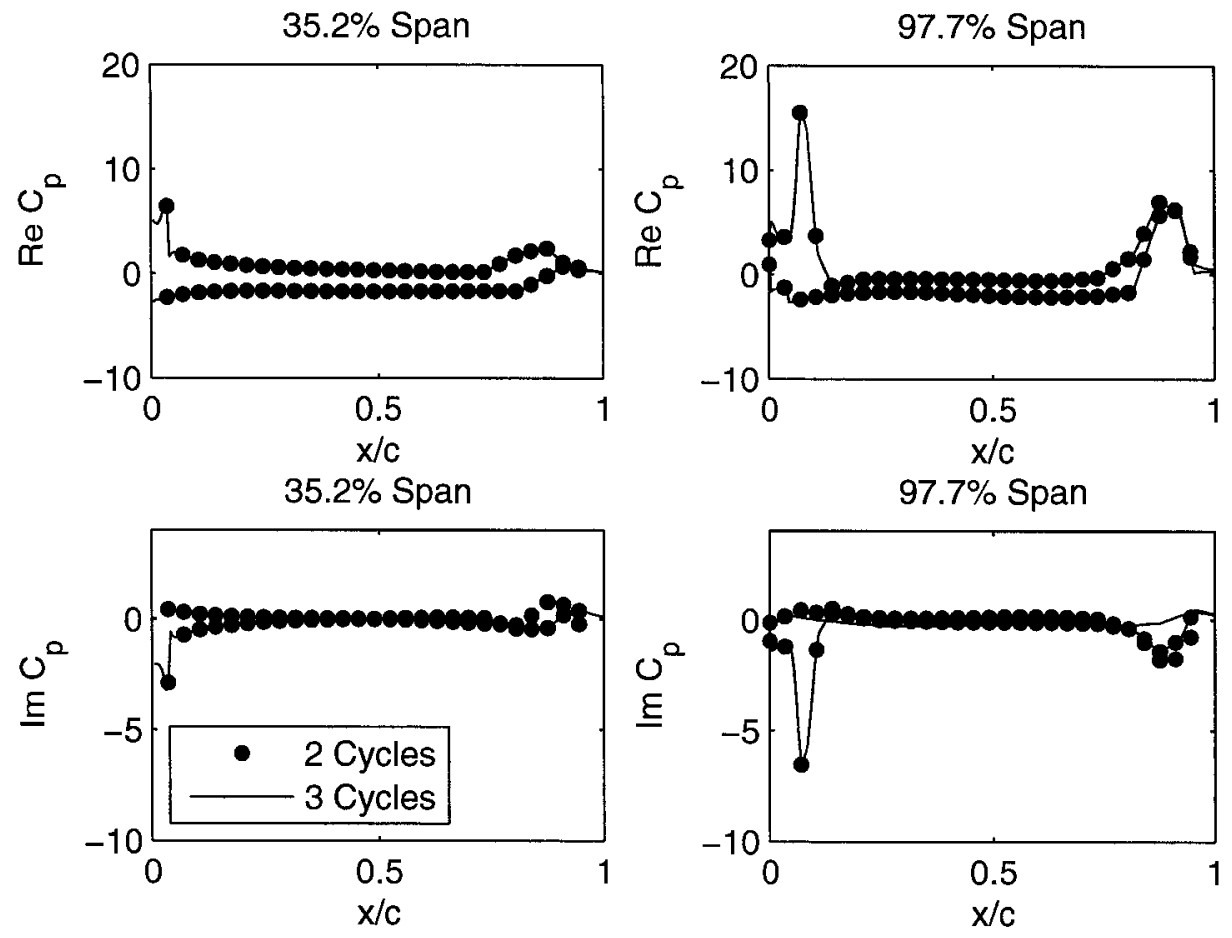

(a) Euler
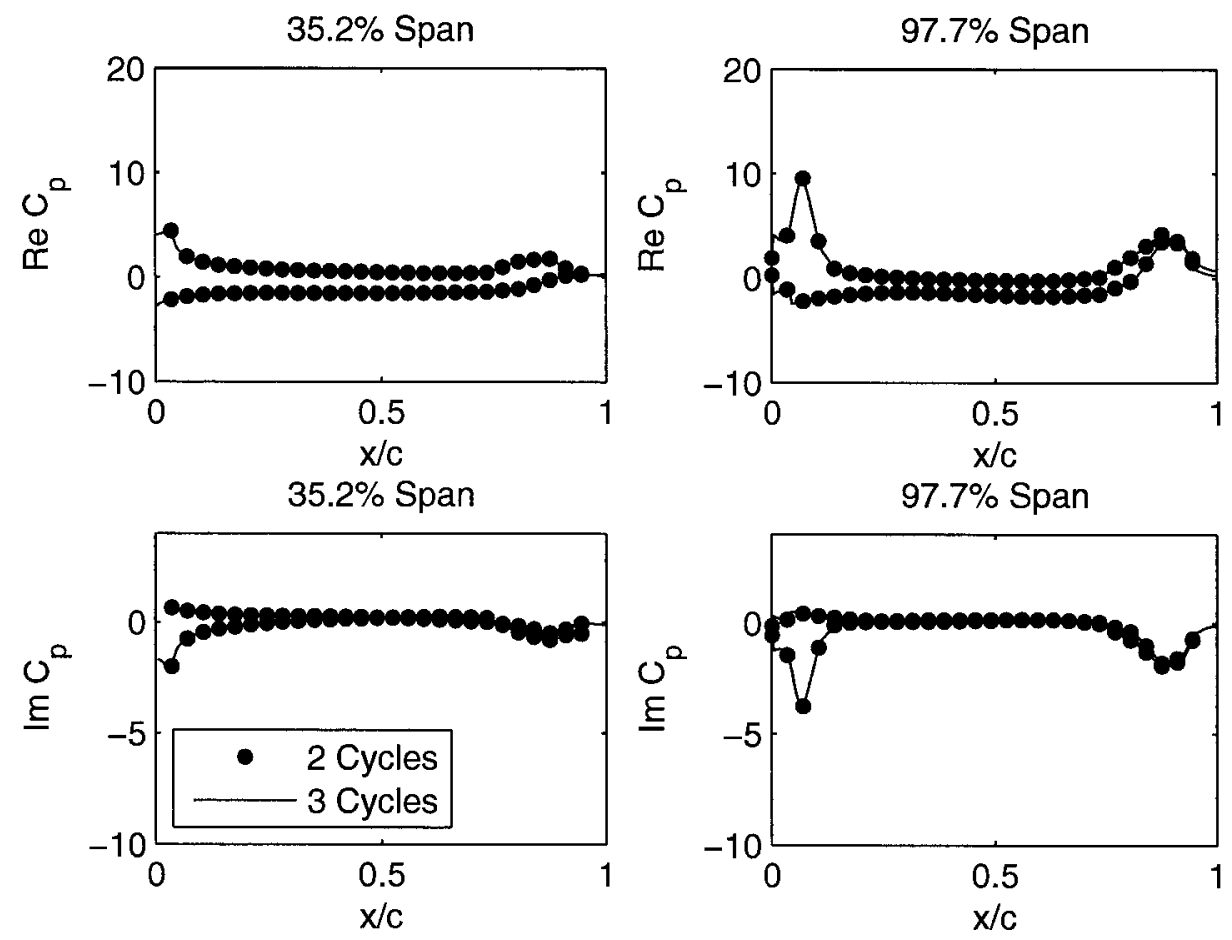

(b) Navier-Stokes

Figure 5.5: Real and imaginary $C_{p}$ over F-5 wing at $M_{\infty}=0.95$ for 2 and 3 cycles. 

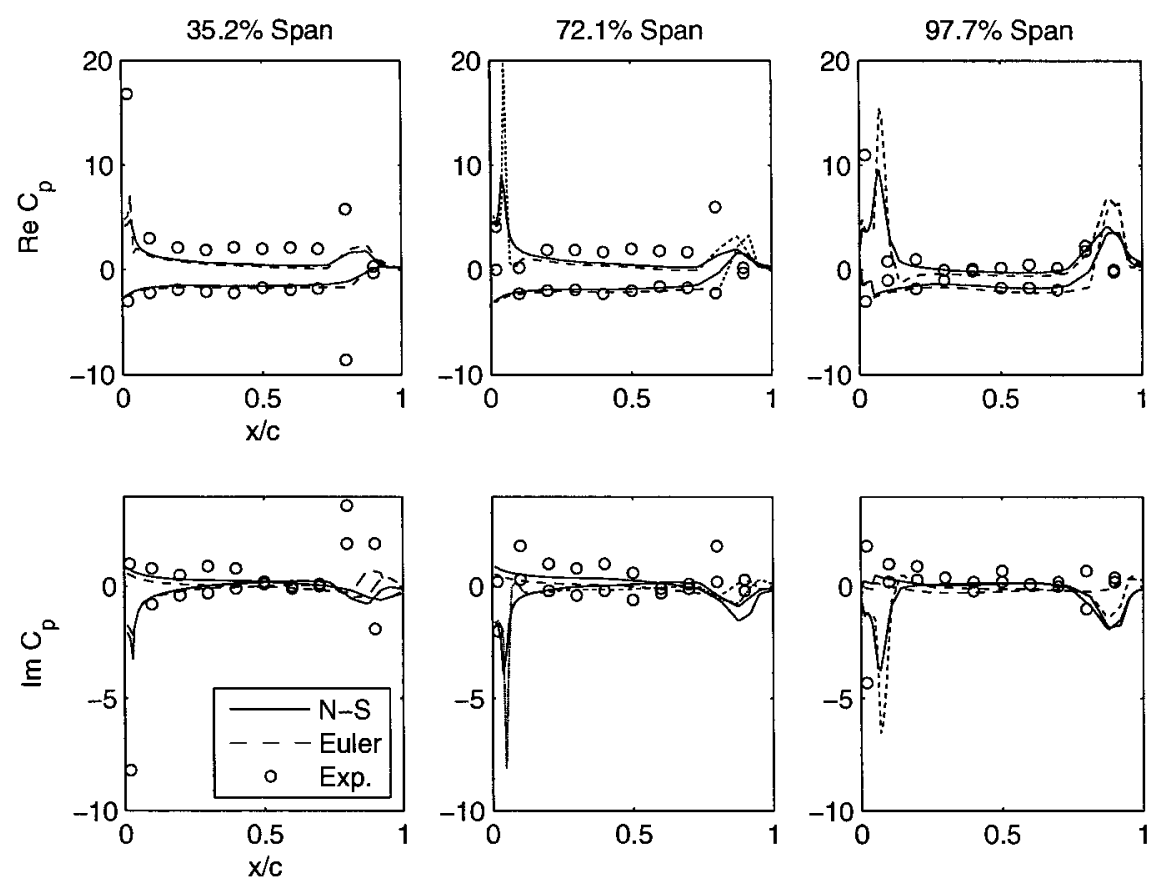

Figure 5.6: Real and imaginary $C_{p}$ over F-5 wing at $M_{\infty}=0.95$.
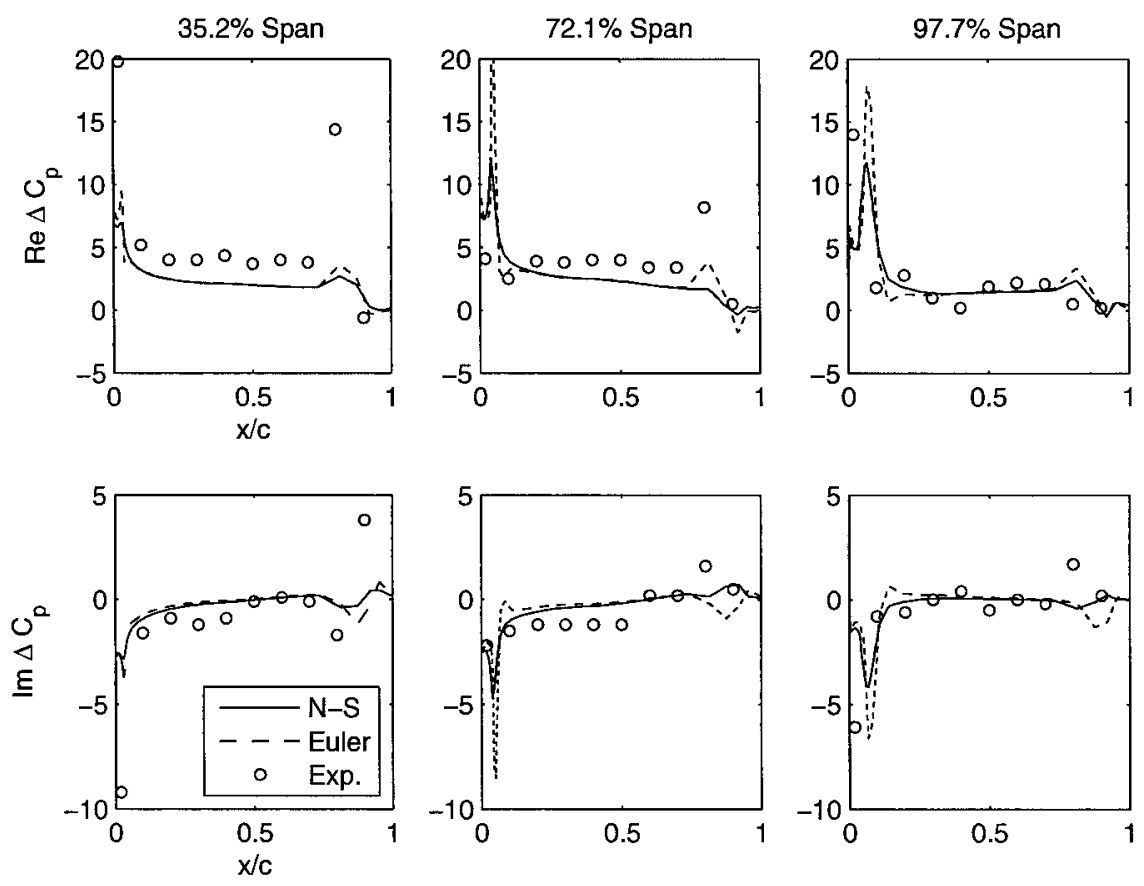

Figure 5.7: Unsteady $\Delta C_{p}$ over F-5 wing at $M_{\infty}=0.95$. 
drilled through the wing to reduce its stiffness. Flutter speed coefficients, $U_{F}$, for Mach numbers in the range of 0.338 to 1.141 were reported. Refer to Reference [51] for the description of the wing geometry, test conditions and the experimental results.

The flutter speed coefficient is expressed as

$$
U_{F}=\frac{U_{\infty}}{b_{s} \omega_{\alpha} \mu^{1 / 2}}
$$

where $U_{\infty}$ is the freestream velocity at flutter, $b_{s}$ is the wing semispan, $\omega_{\alpha}=39.44$ $\mathrm{Hz}$ is the frequency of the first torsional mode (shown in Chapter 5.2.3) and $\mu=$ $m /\left(\rho_{\infty} V\right)$ where $m=1.693 \mathrm{~kg}$ is the mass of the wing, $V=0.130 \mathrm{~m}^{2}$ is related to the volume of the wing and $\rho_{\infty}$ is the freestream density at flutter.

Flutter speed coefficients will be calculated for: (1) time marching simulations using the Euler and Navier-Stokes equations; (2) uncorrected frequency domain simulations using MSC/NASTRAN and KEDLMPL; and (3) AIC corrected frequency domain simulations using KEDLMPL and PMB CFD data. The flow conditions for the various Mach numbers are shown in Table 5.3. Results will be shown in Chapter 6 . The following sections will describe and validate the AGARD 445.6 wing aerodynamic and structural models.

\subsubsection{CFD Fluid Meshes}

An O-O grid and a $\mathrm{C}-\mathrm{H}$ grid were generated by a proprietary in-house code for use in the Euler and Navier-Stokes calculations, respectively. Medium and coarse grids 
Table 5.3: Flow conditions for AGARD 445.6 wing flutter analysis.

\begin{tabular}{ccc}
\hline \hline Mach Number & Reynolds Number & Density $\left[\mathrm{kg} / \mathrm{m}^{3}\right]$ \\
\hline 0.678 & $1.410 \times 10^{6}$ & 0.2082 \\
0.901 & $0.911 \times 10^{6}$ & 0.0995 \\
0.960 & $0.627 \times 10^{6}$ & 0.0634 \\
0.990 & $0.534 \times 10^{6}$ & 0.0593 \\
1.072 & $0.442 \times 10^{6}$ & 0.0552 \\
\hline \hline
\end{tabular}

were created for both the O-O and the $\mathrm{C}-\mathrm{H}$ grids. The medium O-O grid had 190,000 nodes with 4,453 points on the wing surface. The coarse O-O grid had 27,000 nodes with 1,131 points on the wing surface. The medium $\mathrm{C}-\mathrm{H}$ grid had 324,000 nodes with 2,862 points on the wing surface. The coarse $\mathrm{C}-\mathrm{H}$ grid had 45,000 nodes with 979 points on the wing surface. Refer to Fig. 5.8.

A grid convergence study was conducted for a steady case at zero angle of attack, Mach number of 0.96 and a Reynolds number of $4.51 \times 10^{5}$ based on mean aerodynamic chord. The results for the Euler and Navier-Stokes solutions are shown in Fig. 5.9 for four wing stations. Convergence history for the medium grids is shown in Fig. 5.10. Both the medium and coarse grids show good agreement for the respective topologies. The Euler and Navier-Stokes solutions show minor disagreement near the trailing edge of the wing due to the difference between the grid topologies.

Therefore, the coarse $\mathrm{O}-\mathrm{O}$ and $\mathrm{C}-\mathrm{H}$ grids were employed in the present investigations. 


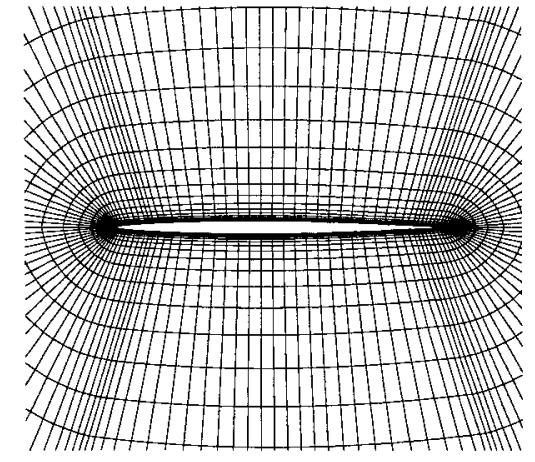

(a) Euler Root Section

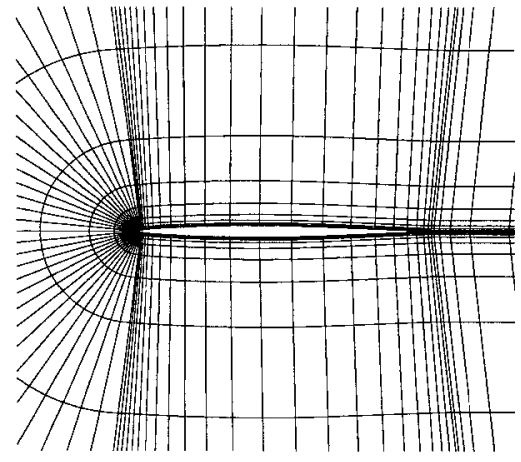

(b) Navier-Stokes Root Section

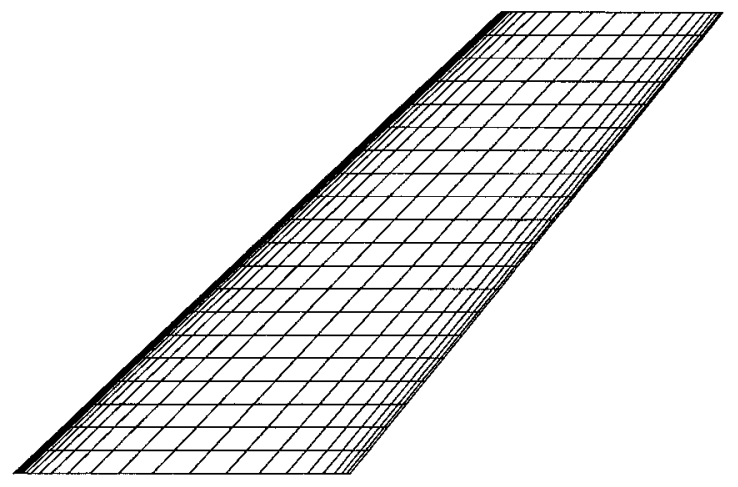

(c) Euler and Navier-Stokes Upper Wing Surfaces

Figure 5.8: Medium density AGARD 445.6 CFD meshes. 

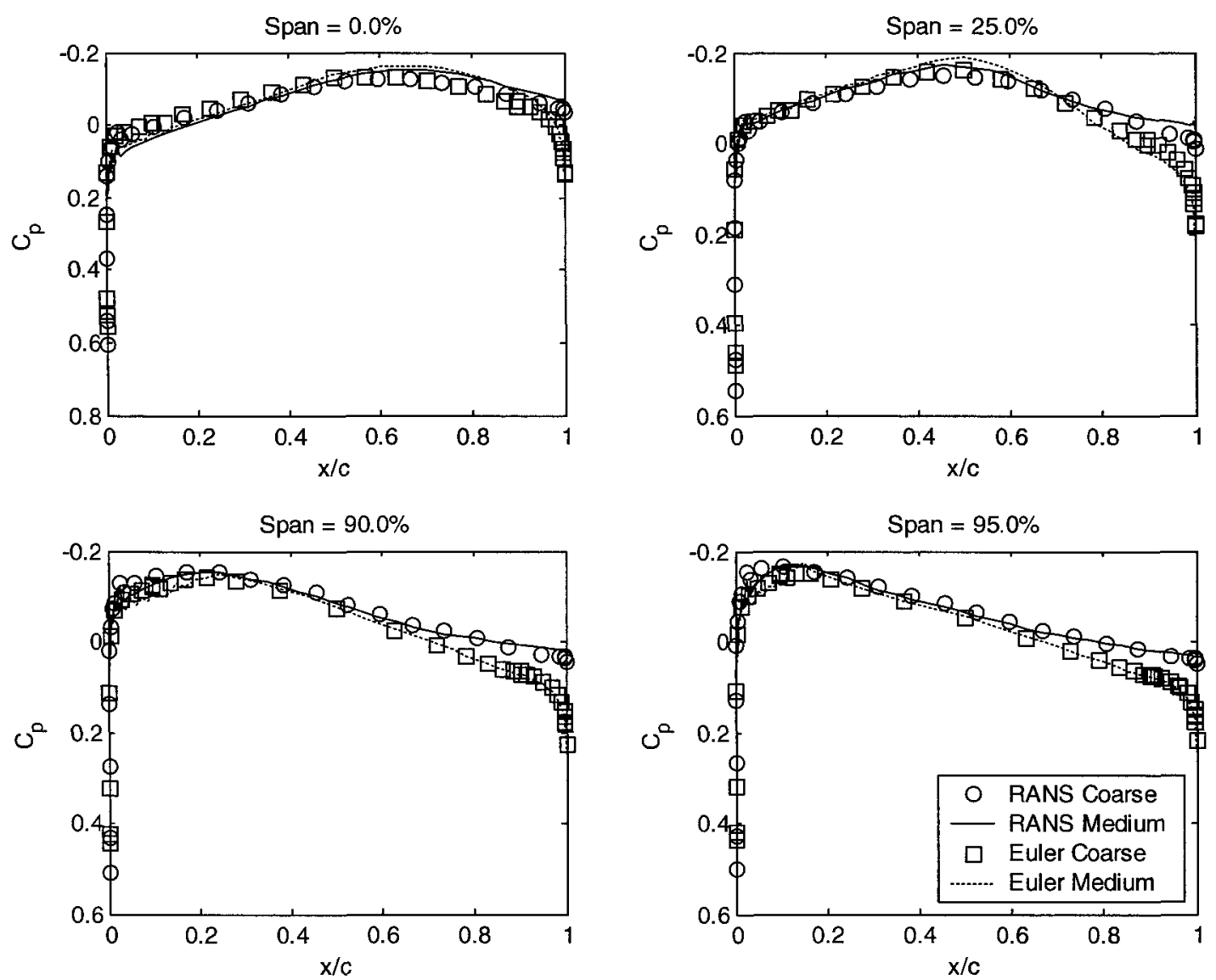

Figure 5.9: Steady $C_{p}$ over AGARD 445.6 upper wing surface at $M_{\infty}=0.96$. 


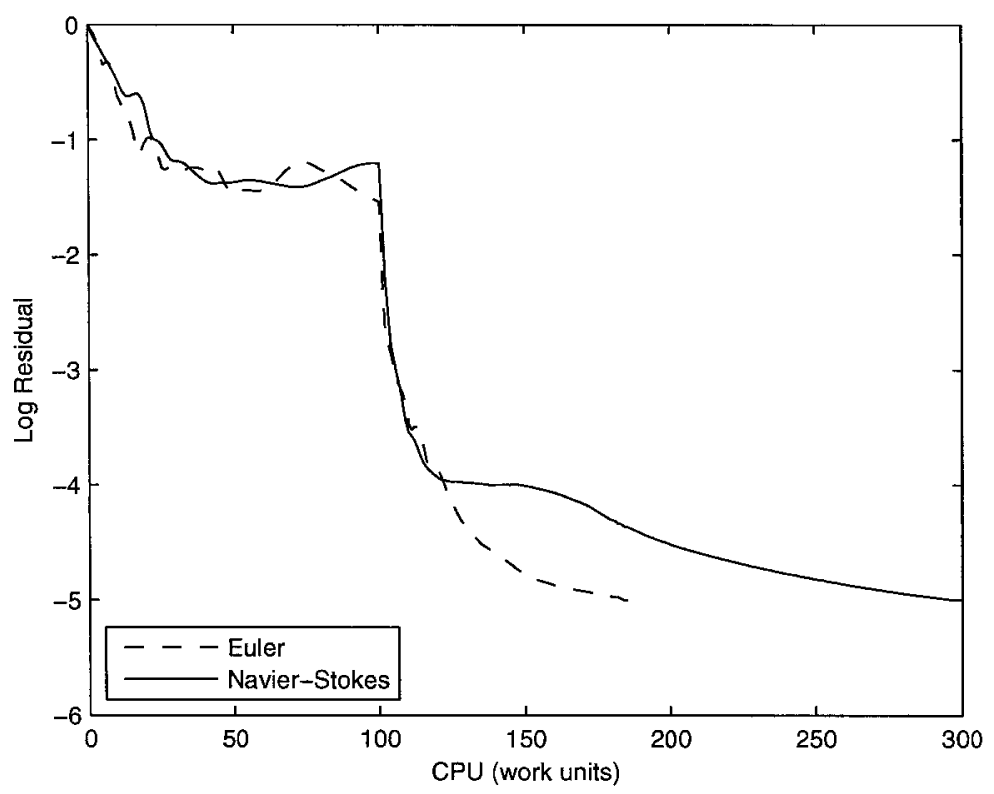

Figure 5.10: Steady state convergence for medium density AGARD 445.6 wing meshes at $M_{\infty}=0.96$.

\subsubsection{DLM Mesh}

A linear aerodynamic model was created for use in the DLM simulations employing MSC/NASTRAN and KEDLMPL. The wing was modelled by panels and the lifting surfaces were assumed to lie parallel to the flow. Note, the DLM does not account for thickness effects of the lifting surfaces and assumes the undisturbed flow is uniform or varying harmonically. The model consisted of 21 points in the spanwise direction and 21 points in the chordwise direction. The spanwise and chordwise point distributions were uniformly set in order to maintain near unity box ratios as recommended by Reference [52]. 


\subsubsection{Structural Model}

The linear structural model was created in MSC/NASTRAN using the model parameters in the aeroelastic optimization study by Reference [53]. This model was selected for comparative purposes as it was also used by References [4], [49] and [50] for time marching studies. The wing was modelled with plate elements as a single layer orthotropic material. The model consisted of 231 nodes and 200 elements. Refer to Fig. 5.11. The thickness distribution was governed by the airfoil shape. The material properties used were $E_{1}=3.1511 \mathrm{GPa}, E_{2}=0.4162 \mathrm{GPa}, \nu=0.31, G=0.4392 \mathrm{GPa}$ and $\rho=381.98 \mathrm{~kg} / \mathrm{m}^{3}$ where $E_{1}$ and $E_{2}$ are the moduli of elasticity in the longitudinal and lateral directions, $\nu$ is Poisson's ratio, $G$ is the shear modulus in each plane and $\rho$ is the wing density. Table 5.4 compares the measured and calculated first four fundamental modes and Fig. 5.12 shows the calculated mode shapes.

Table 5.4: Comparison of modal frequencies for AGARD 445.6 wing.

\begin{tabular}{lcccc}
\hline \hline & Mode 1 [Hz] & Mode 2 [Hz] & Mode 3 [Hz] & Mode 4 [Hz] \\
\hline Experiment [51] & 9.60 & 38.10 & 50.70 & 98.50 \\
Reference [4] & 9.67 & 36.87 & 50.26 & 90.00 \\
Reference [53] & 9.63 & 37.12 & 50.50 & 89.94 \\
Reference [54] & 9.69 & 37.84 & 51.00 & 92.36 \\
Calculated & 9.46 & 39.44 & 49.71 & 94.39 \\
\hline \hline
\end{tabular}

References [4] and [54] employed the density and model definition of Reference [53]. Reference [4] reported a match in the model mass, suggesting that the thickness of the plate elements were adjusted in order to force match the model mass. Reference [54], 
however, does not report the mass.

Using the density and model definition of Reference [53] resulted in a wing mass of $1.693 \mathrm{~kg}, 9 \%$ lighter than the Reference [53] model (mass equal to the experimental model). The modal frequencies and shapes showed good comparison between all three models and thus, the density was not adjusted to match the wing mass.

The linear structural model was used for both the Euler and Navier-Stokes time marching as well as the frequency domain simulations. The addition of structural damping is not included for the time marching or KEDLMPL solutions. Thus, for comparative purposes, the structural damping for the MSC/NASTRAN model was set to zero. 


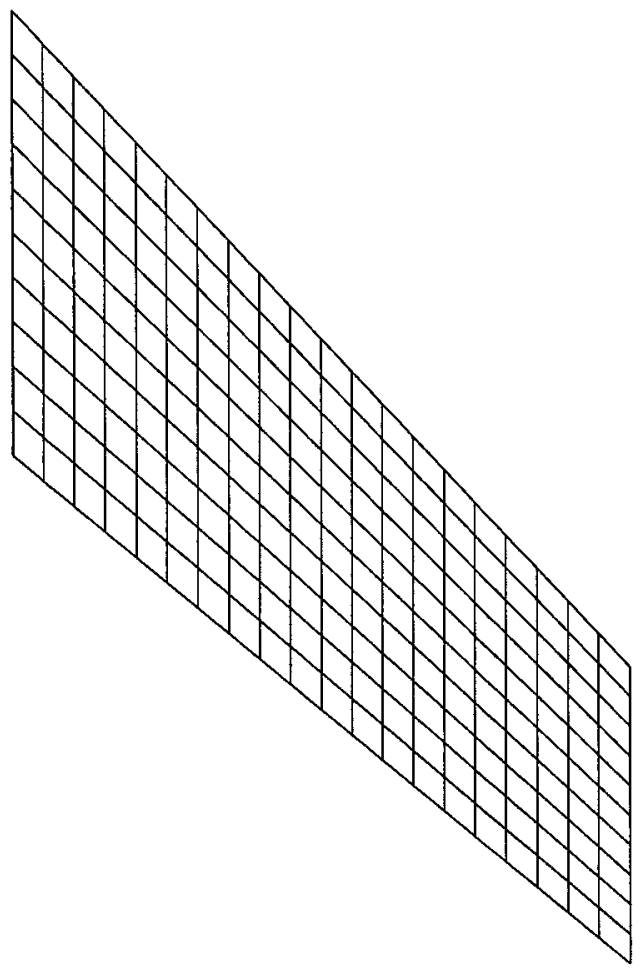

Figure 5.11: AGARD 445.6 structural model. 


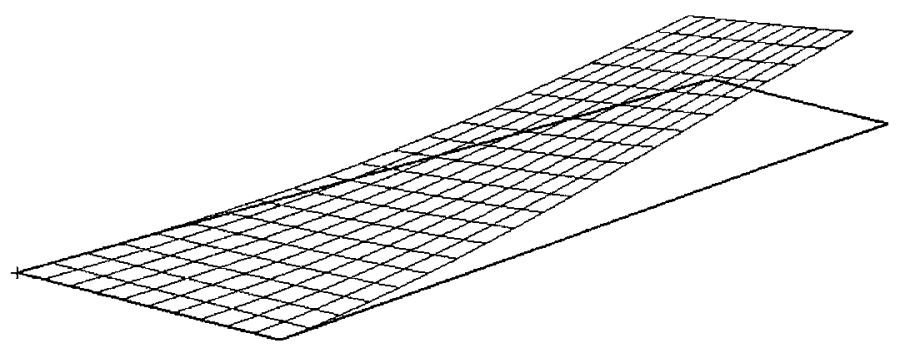

(a) Mode 1 (1-B)

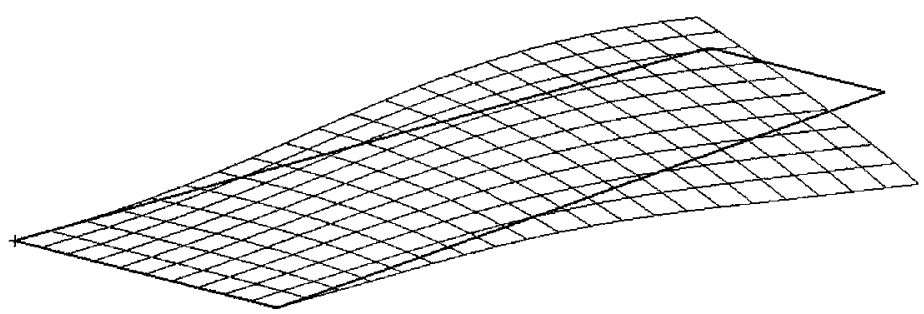

(b) Mode 2 (1-T)

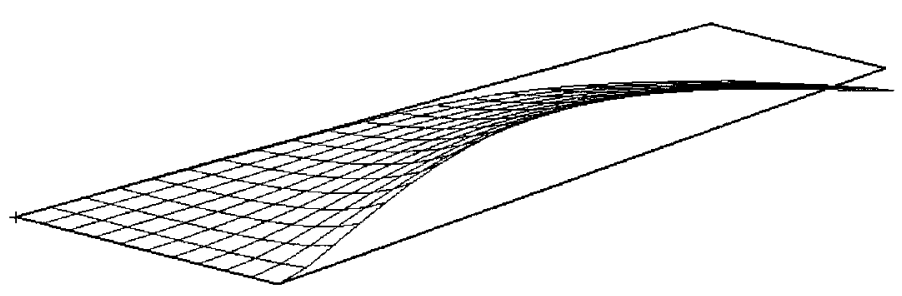

(c) Mode $3(2-\mathrm{B})$

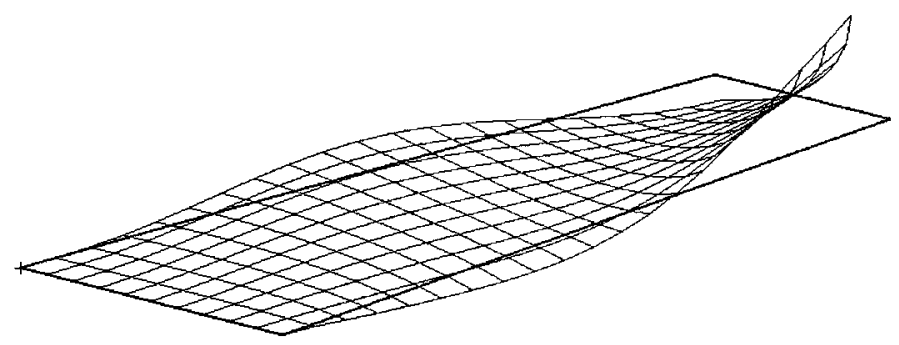

(d) Mode 4 (2-T)

Figure 5.12: Calculated AGARD 445.6 wing mode shapes. 


\section{Chapter 6}

\section{Results}

\subsection{Uncorrected Frequency Domain Results}

Based on DLM aerodynamic theory and the KE-method, uncorrected frequency domain results were obtained using MSC/NASTRAN and KEDLMPL. Refer to Chapter 2 for the frequency domain formulation and to Chapter 5 for the description of the test case.

Flutter boundaries for the AGARD 445.6 weakened wing are shown in Fig. 6.1 at $M_{\infty}=0.678$ to $M_{\infty}=0.990$. The MSC/NASTRAN and KEDLMPL solutions produced significantly higher flutter boundaries in comparison to the experimental results. These programs also failed to predict the transonic dip located after $M_{\infty}=0.90$. Instead, MSC/NASTRAN and KEDLMPL produced erroneous linearly decreasing flutter boundaries.

KEDLMPL produced a boundary similar to MSC/NASTRAN, the code employed by industry for flutter certification. The variance in flutter speed between KEDLMPL 
and MSC/NASTRAN was unexpected as both programs utilized the same aerodynamic and structural models as well as the same implementation of the DLM. Further investigation was not possible as the source code of MSC/NASTRAN was unavailable.

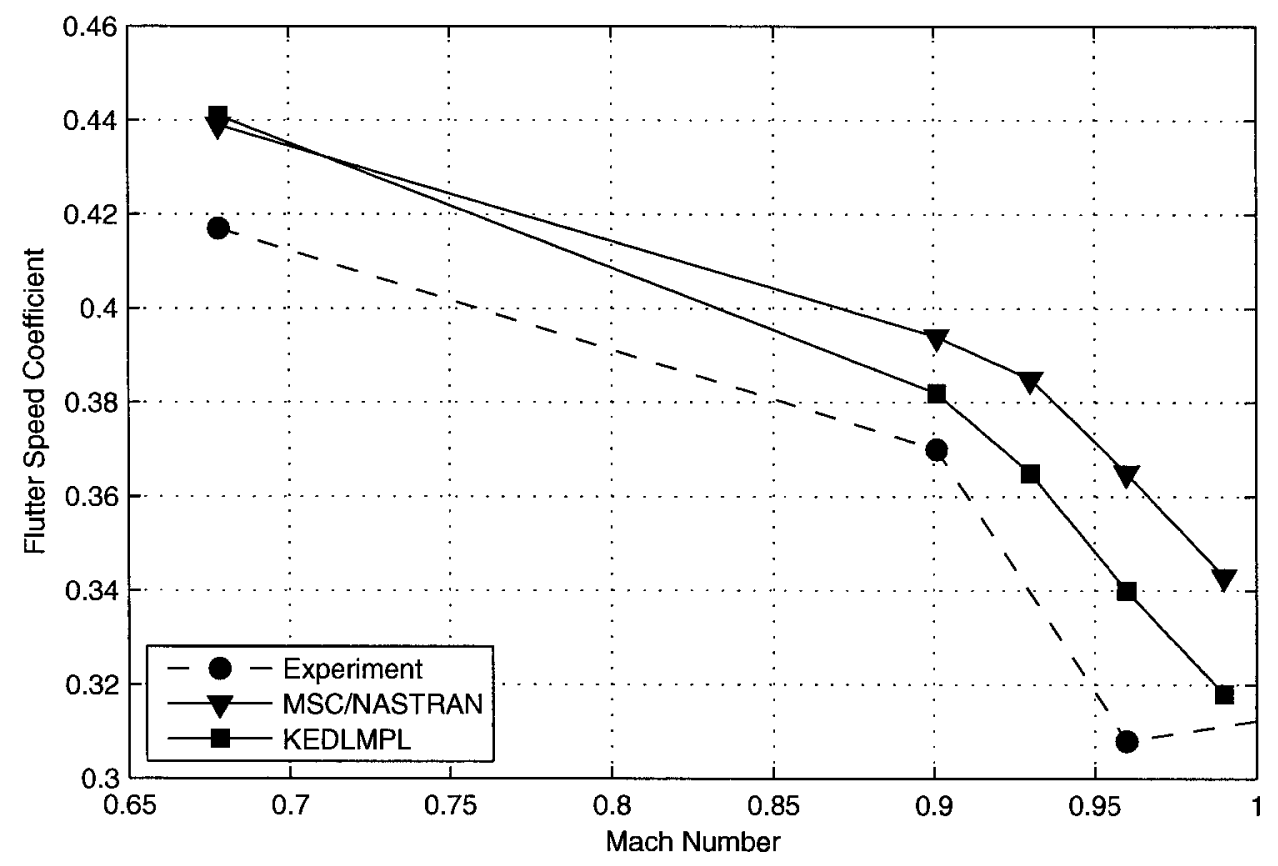

Figure 6.1: Uncorrected frequency domain flutter boundaries for AGARD 445.6 wing.

The mechanism of flutter for all tested Mach numbers was between modes 1 (first bending) and 2 (first torsion). This mechanism can be observed in the velocitydamping $(\mathrm{V}-\mathrm{g})$ and velocity-frequency (V-f) plots. Refer to Fig. 6.2 for the $\mathrm{V}$-g and V-f plots at $M_{\infty}=0.678$

The uncorrected frequency domain simulations were conducted on a machine with a single $2.8 \mathrm{GHz}$ processor. The computational time required to calculate each flutter data point for MSC/NASTRAN and KEDLMPL was 5 minutes and 4 minutes, 
respectively.

\subsection{Time Marching Results}

Time marching simulations based on the Euler and Navier-Stokes equations were conducted using PMB. Refer to Chapter 4 for the time marching formulation.

In these simulations, the structure was given a small initial dimensionless velocity in the first mode. The subsequent time evolution of the modal response was then calculated in order to determine whether the perturbation had grew or had decayed. If the perturbation decayed, a new simulation was performed using an increased freestream density. This process was repeated until flutter occurred, i.e., when the perturbation grew.

\subsubsection{Grid Density and Fluid Model Effect on Flutter Speed}

The comparison of the time marching solutions at $M_{\infty}=0.96$ (near the bottom of the transonic dip) to various published grid densities and fluid models is shown in Table 6.1. The results of Reference [50] show a $6 \%$ downward trend in the flutter speed between the medium and coarse grids. The current results show an upward trend ( $6 \%$ for Navier-Stokes, $4 \%$ for Euler) similar to the results of Reference [49].

The time marching simulations were conducted on a Beowulf cluster consisting of four machines with $3.2 \mathrm{GHz}$ processors. The computational time required to calculate each flutter data point using the Euler equations with the coarse O-O grid was 4 hours. 

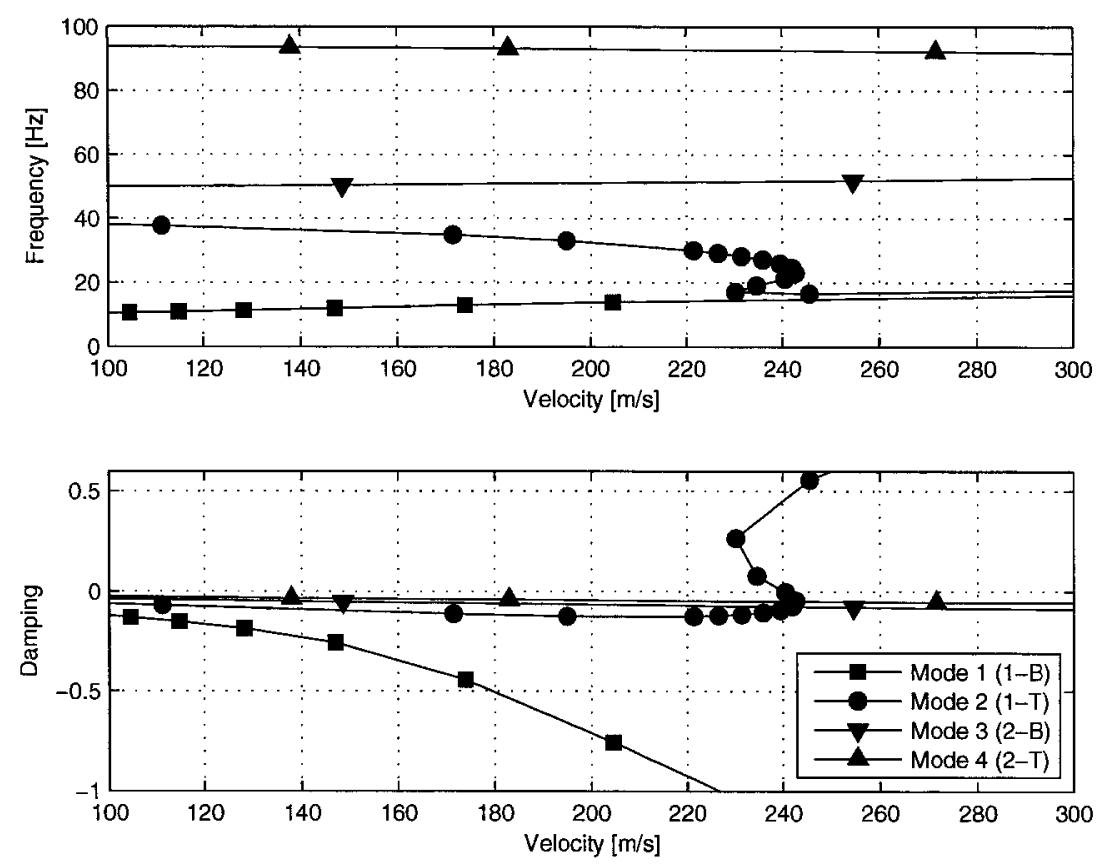

(a) MSC/NASTRAN
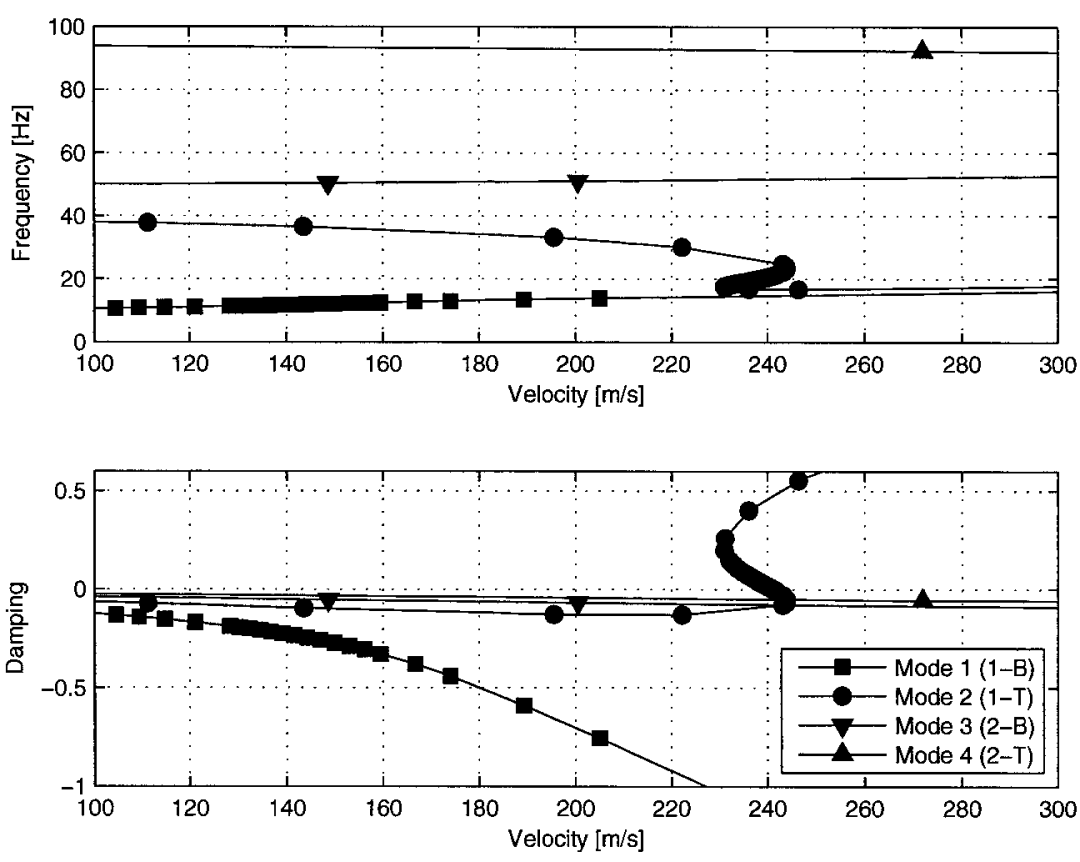

(b) KEDLMPL

Figure 6.2: Uncorrected frequency domain V-f and V-g plots at $M_{\infty}=0.678$. 
The computational time required using the Navier-Stokes equations with coarse C-H grid was 6 hours.

The coarse grids showed good agreement with the medium grids and required approximately three times less computational time. Therefore, the coarse grids were employed to determine the AGARD 445.6 wing flutter boundary.

Table 6.1: Comparison of flutter speed coefficients at Mach 0.96 for various grids.

\begin{tabular}{cccc}
\hline \hline Reference & Grid Volume & Fluid Model & $U_{F}$ \\
\hline Current & Medium & Navier-Stokes & 0.308 \\
Current & Coarse & Navier-Stokes & 0.327 \\
\hline Current & Medium & Euler & 0.317 \\
Current & Coarse & Euler & 0.330 \\
\hline Reference [49] & Fine & Euler & 0.175 \\
Reference [49] & Medium & Euler & 0.192 \\
Reference [49] & Coarse & Euler & 0.227 \\
\hline Reference [50] & Medium & Navier-Stokes & 0.314 \\
Reference [50] & Medium & Navier-Stokes & 0.304 \\
Reference [50] & Medium & Navier-Stokes & 0.285 \\
\hline \hline
\end{tabular}

\subsubsection{Flutter Boundaries}

Flutter boundaries for time marching simulations including previously published results by Reference [4] are shown in Fig. 6.3. The modal responses of the AGARD 445.6 wing before and after flutter using the Euler and Navier-Stokes equations are shown in Figs. 6.6 to 6.8 .

The coarse grid time domain Euler and Navier-Stokes simulations produced similar flutter speeds and boundary shapes between $M_{\infty}=0.678$ and $M_{\infty}=0.96 \mathrm{in}$ 


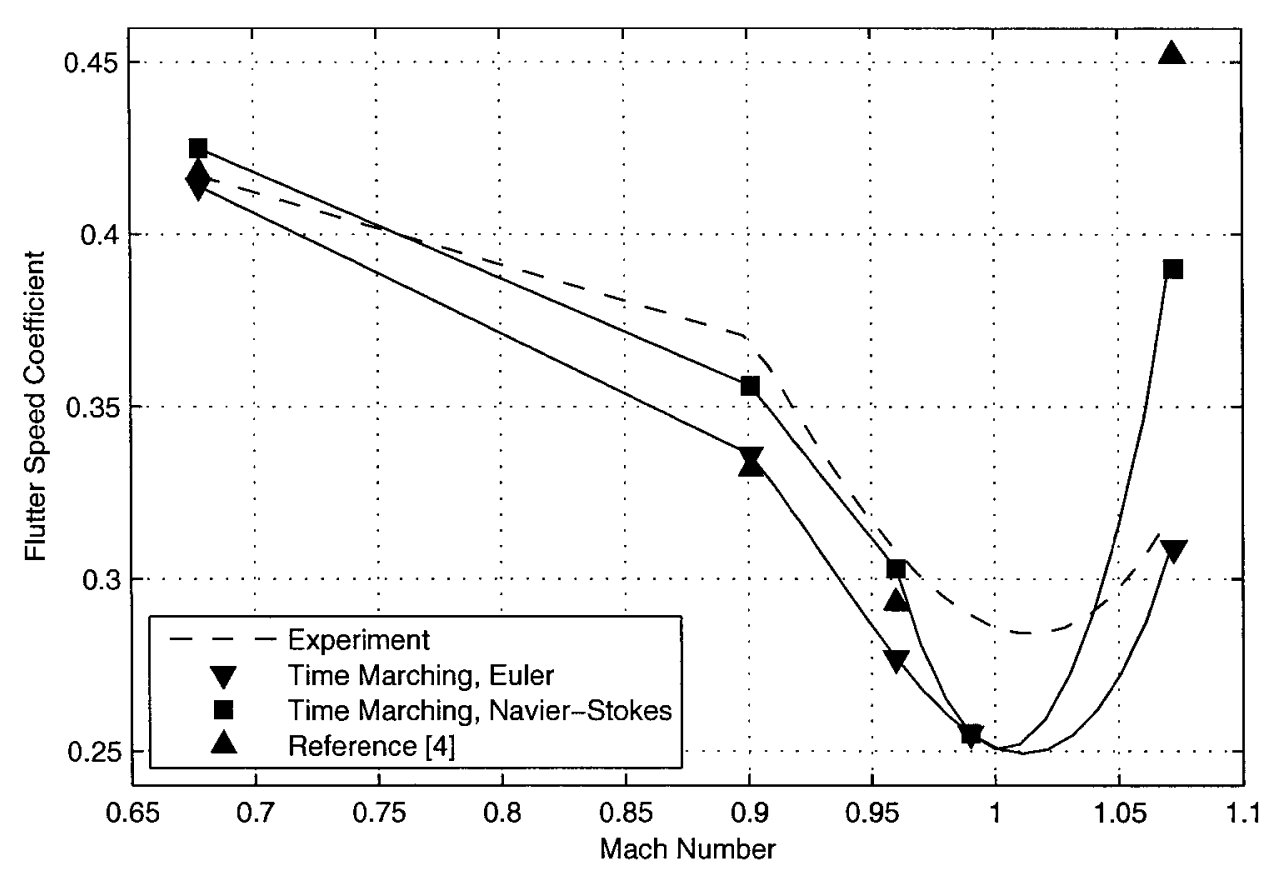

Figure 6.3: Time marching flutter boundaries.

comparison to the experimental results. The time domain boundaries, however, are approximately $6 \%$ lower. This difference may be attributed to the lack of structural damping in the time domain solutions; previous results have shown using a value of structural damping of $0.5 \%$ will shift the boundary towards the experimental results [49].

The Euler and Navier-Stokes simulations predicted identical values for the bottom of the transonic dip. The dip is more pronounced in the Navier-Stokes simulation than the Euler simulation and less pronounced than Reference [4]. 

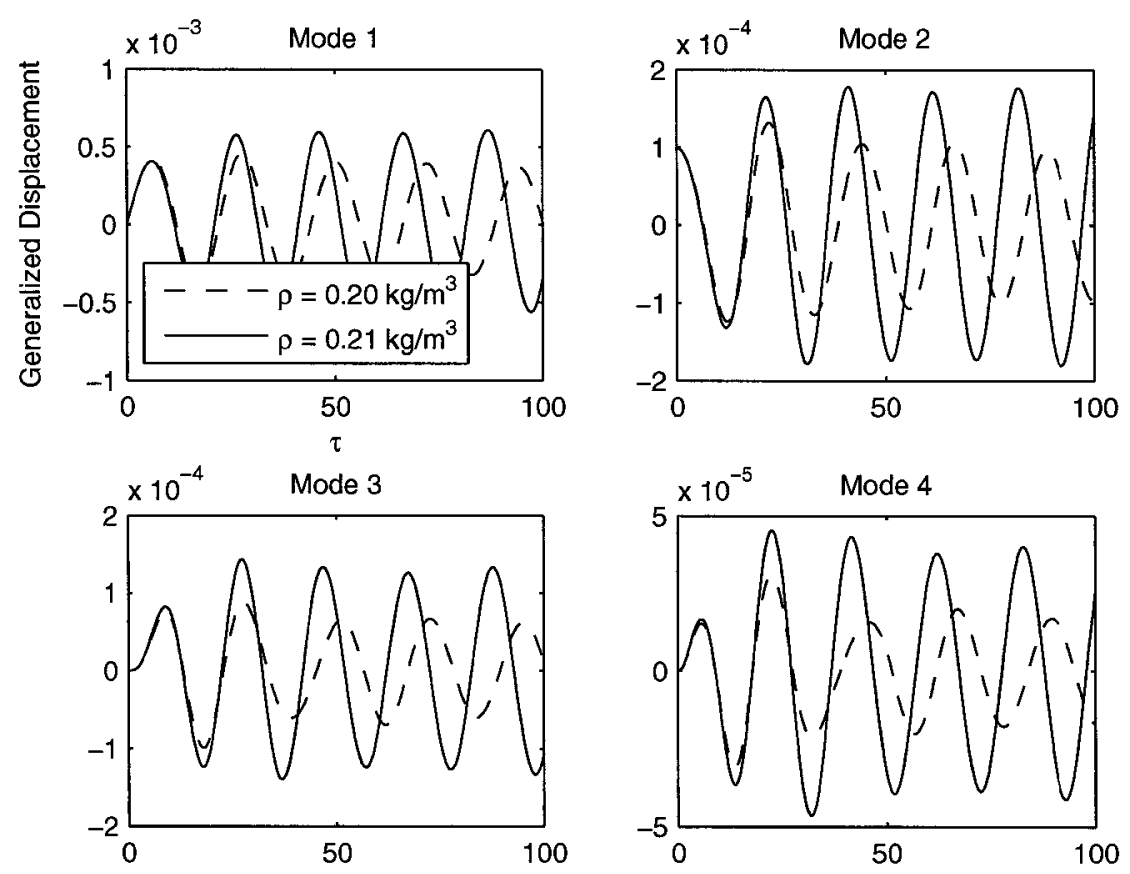

(a) Navier-Stokes
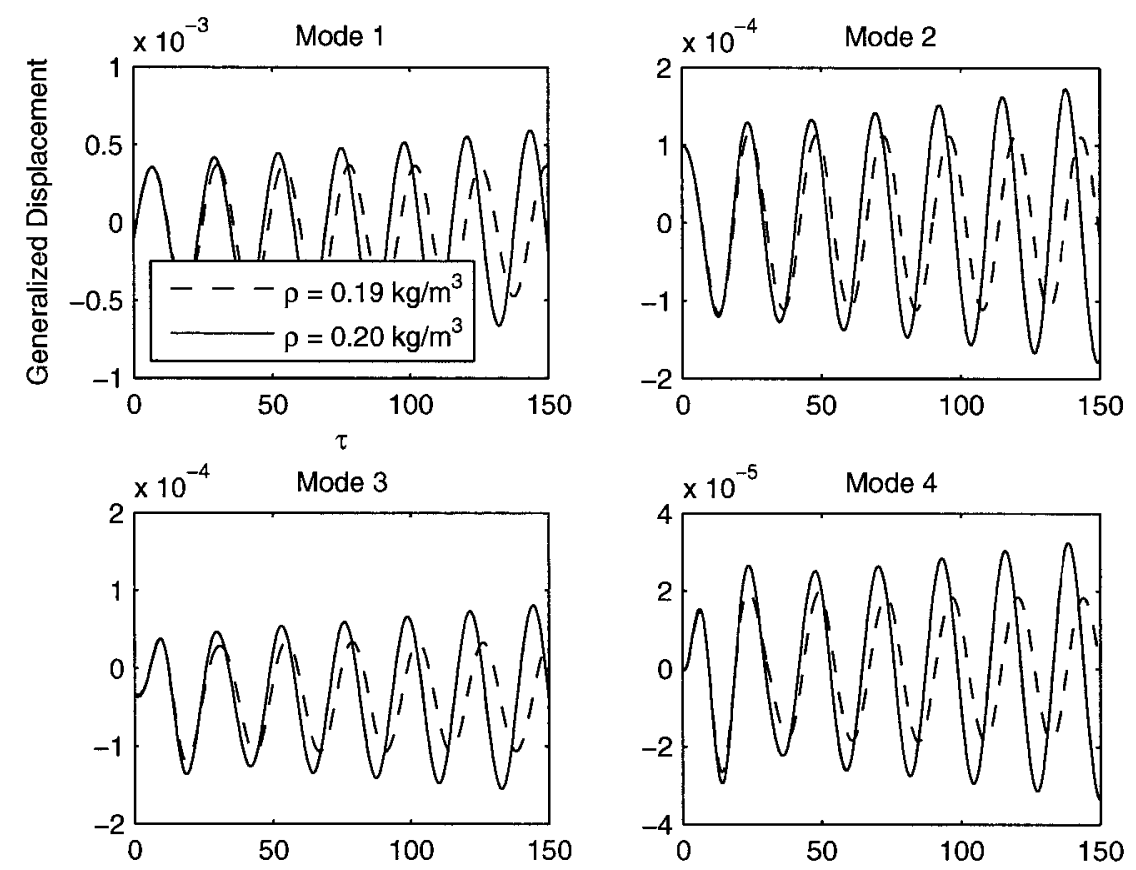

(b) Euler

Figure 6.4: Time marching responses at $M_{\infty}=0.678$. 

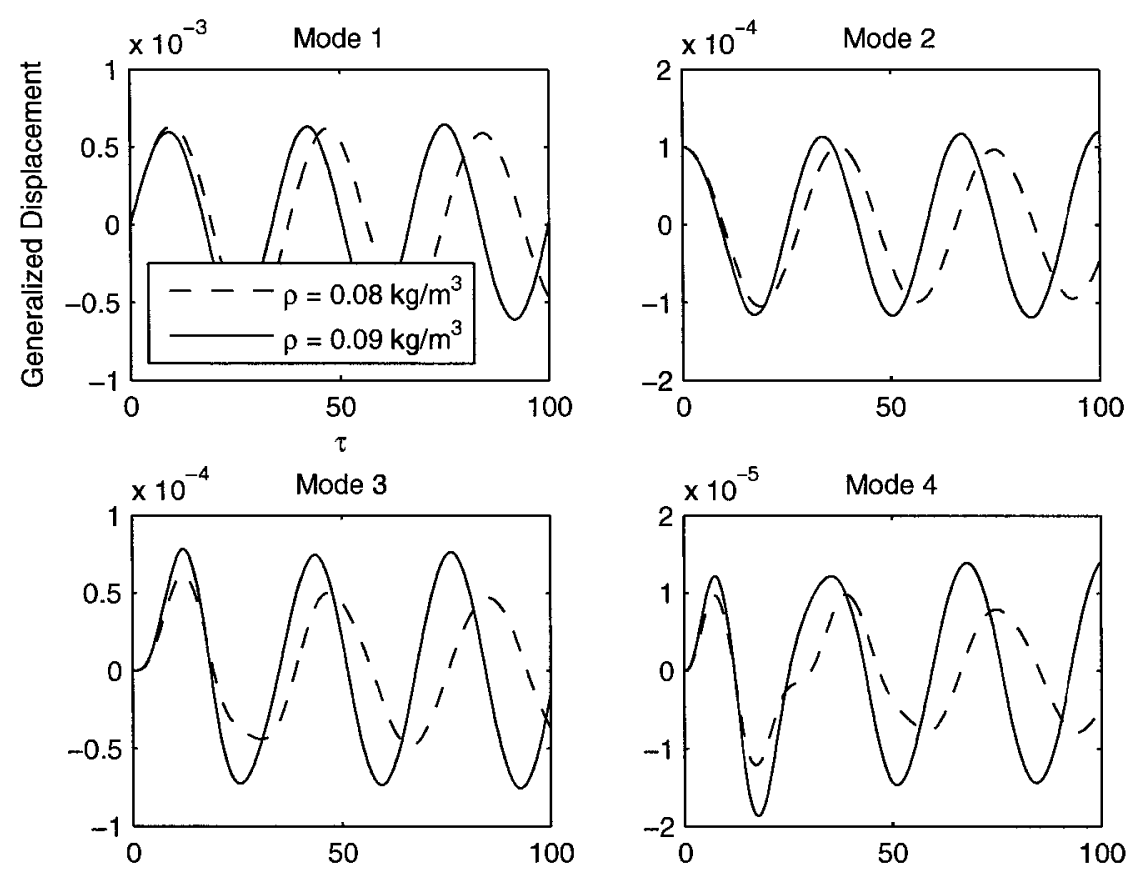

(a) Navier-Stokes
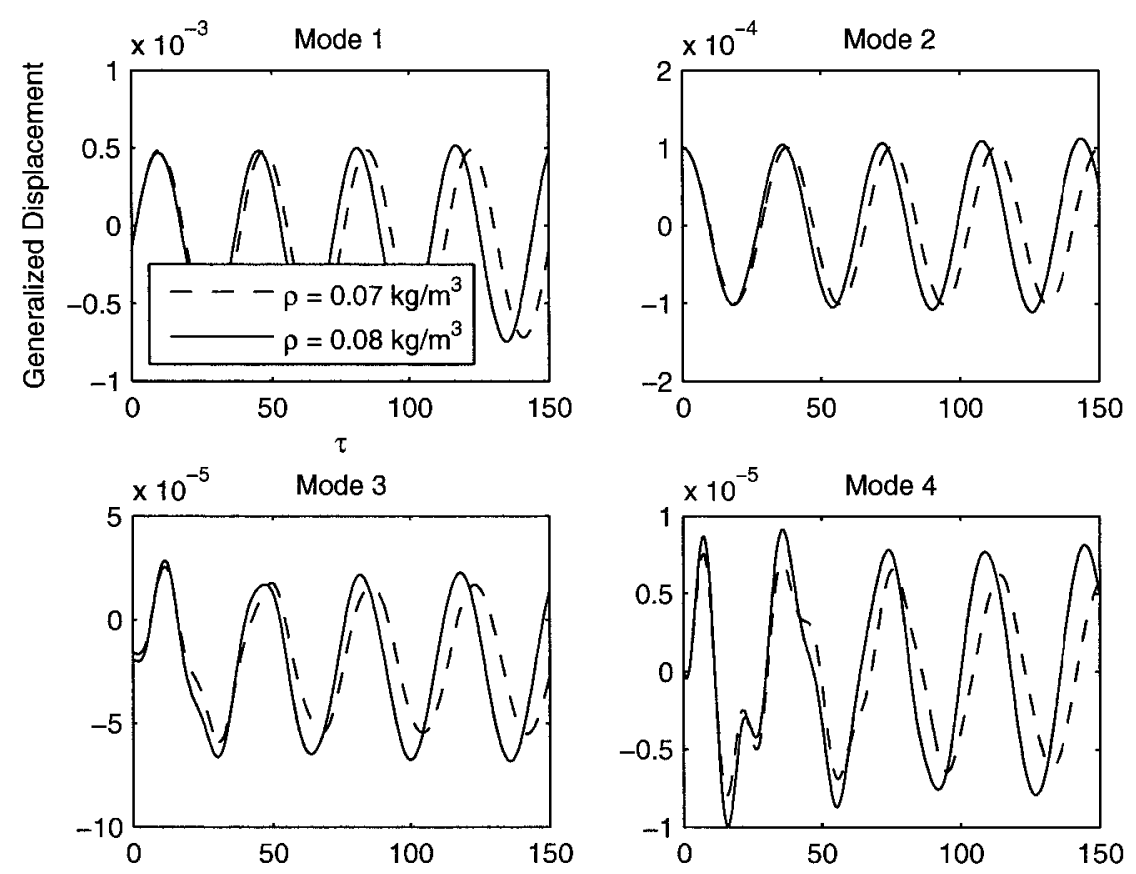

(b) Euler

Figure 6.5: Time marching responses at $M_{\infty}=0.901$. 

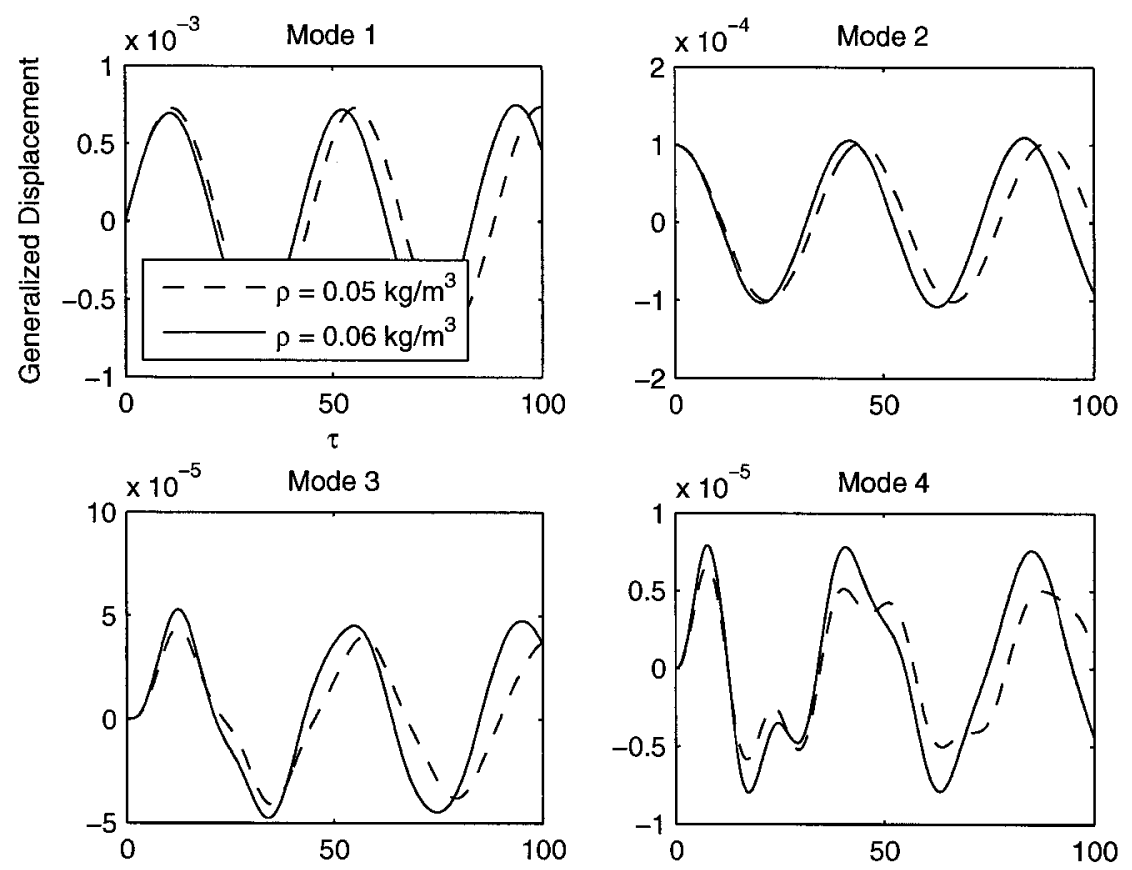

(a) Navier-Stokes
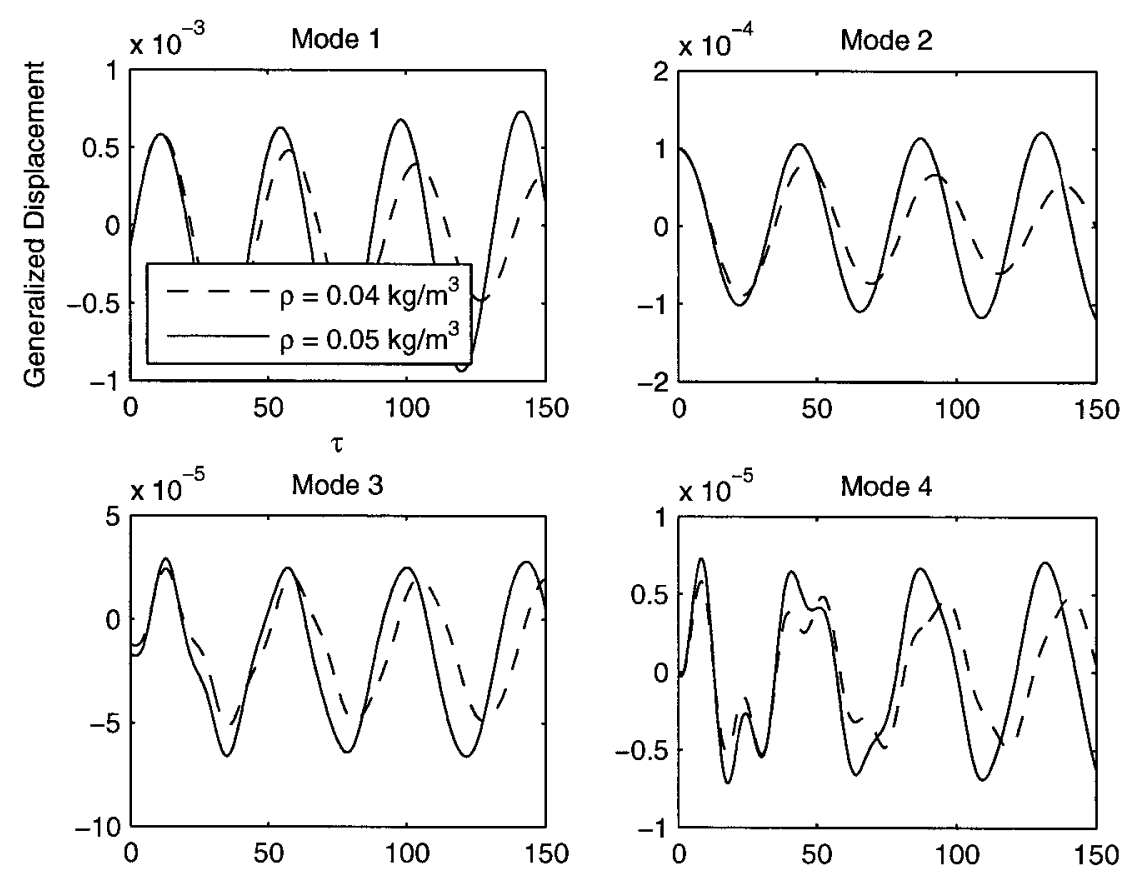

(b) Euler

Figure 6.6: Time marching responses at $M_{\infty}=0.960$. 

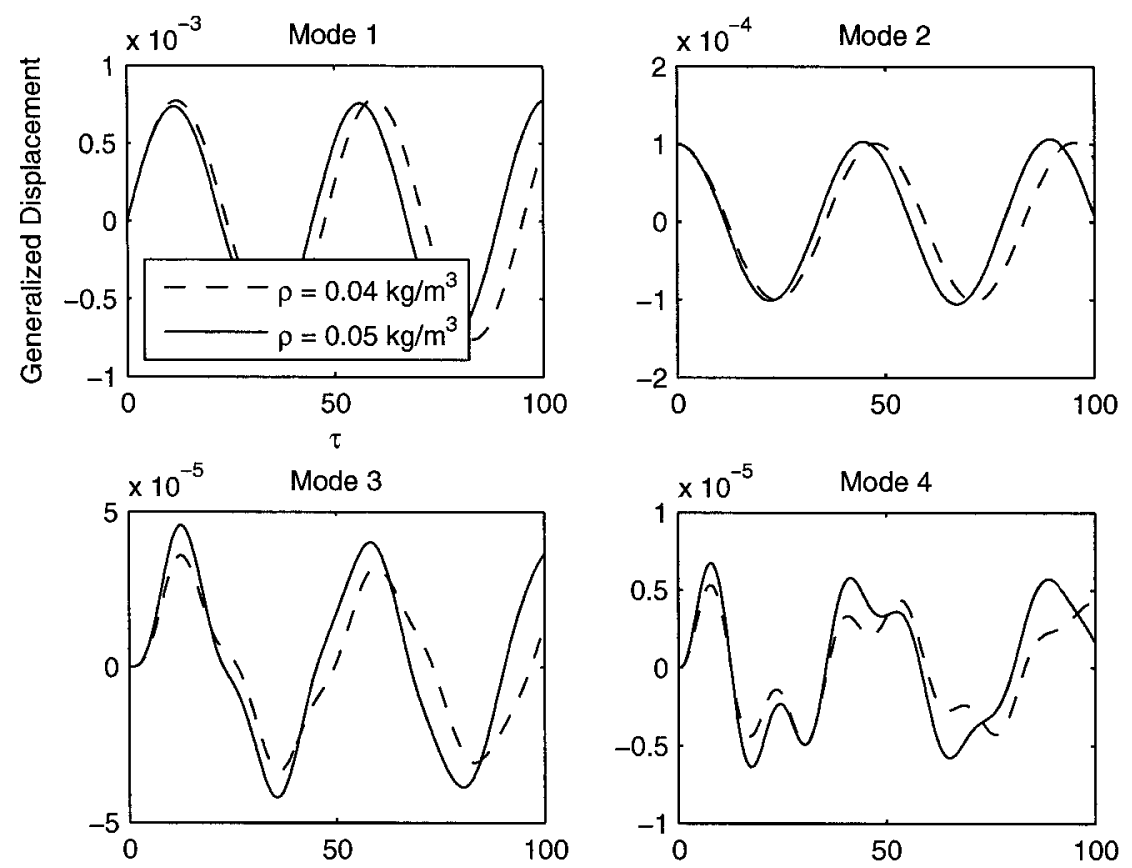

(a) Navier-Stokes
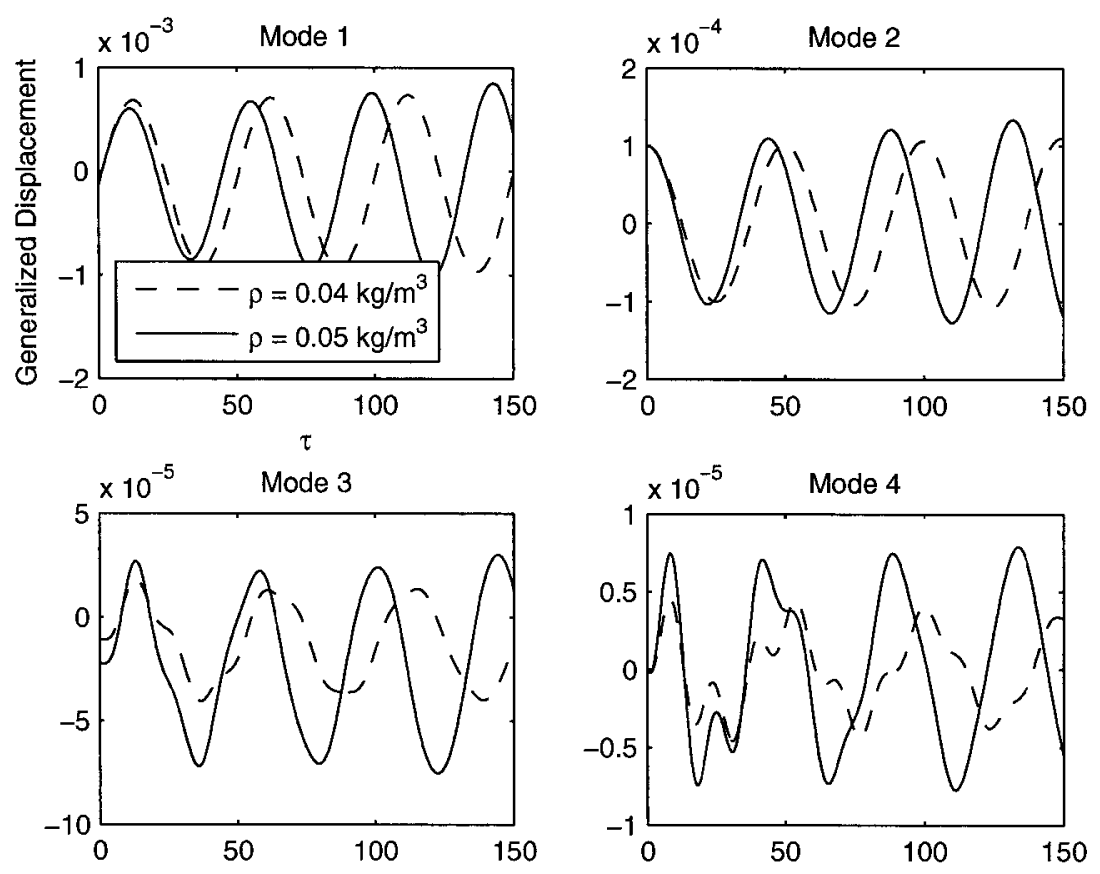

(b) Euler

Figure 6.7: Time marching responses at $M_{\infty}=0.990$. 

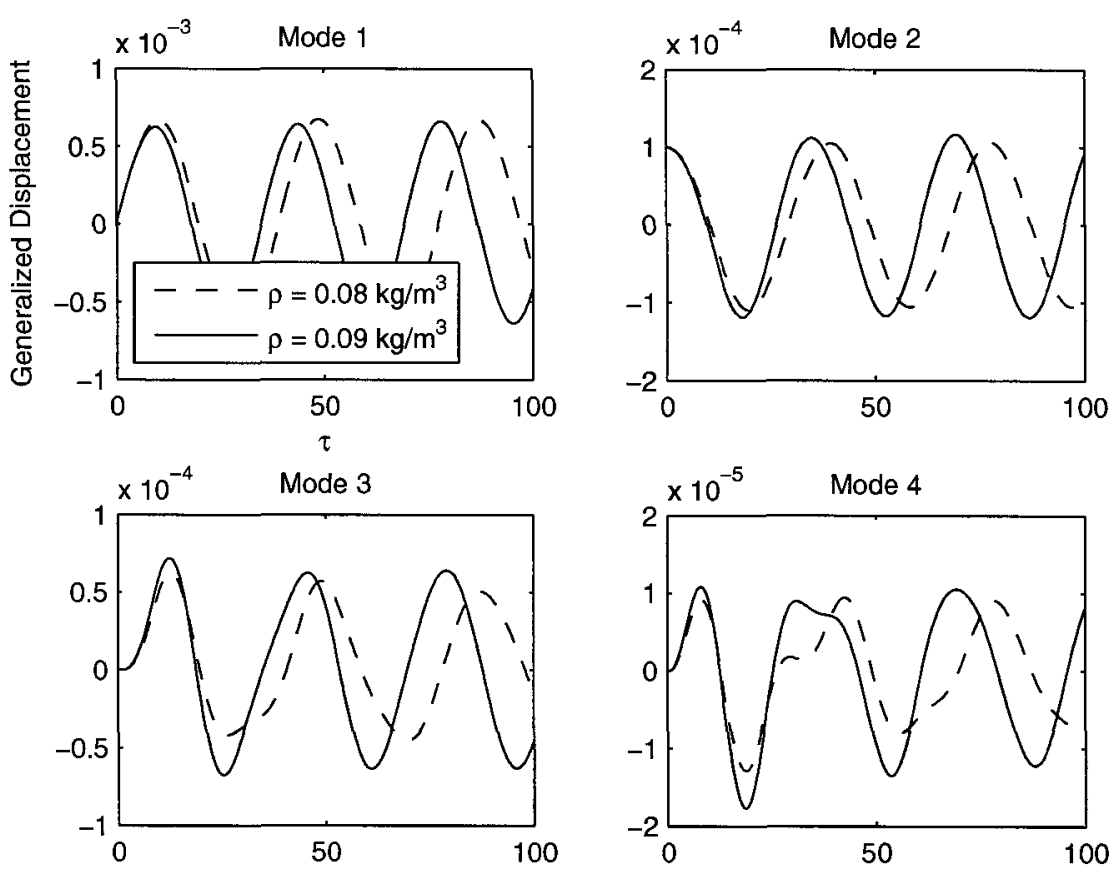

(a) Navier-Stokes
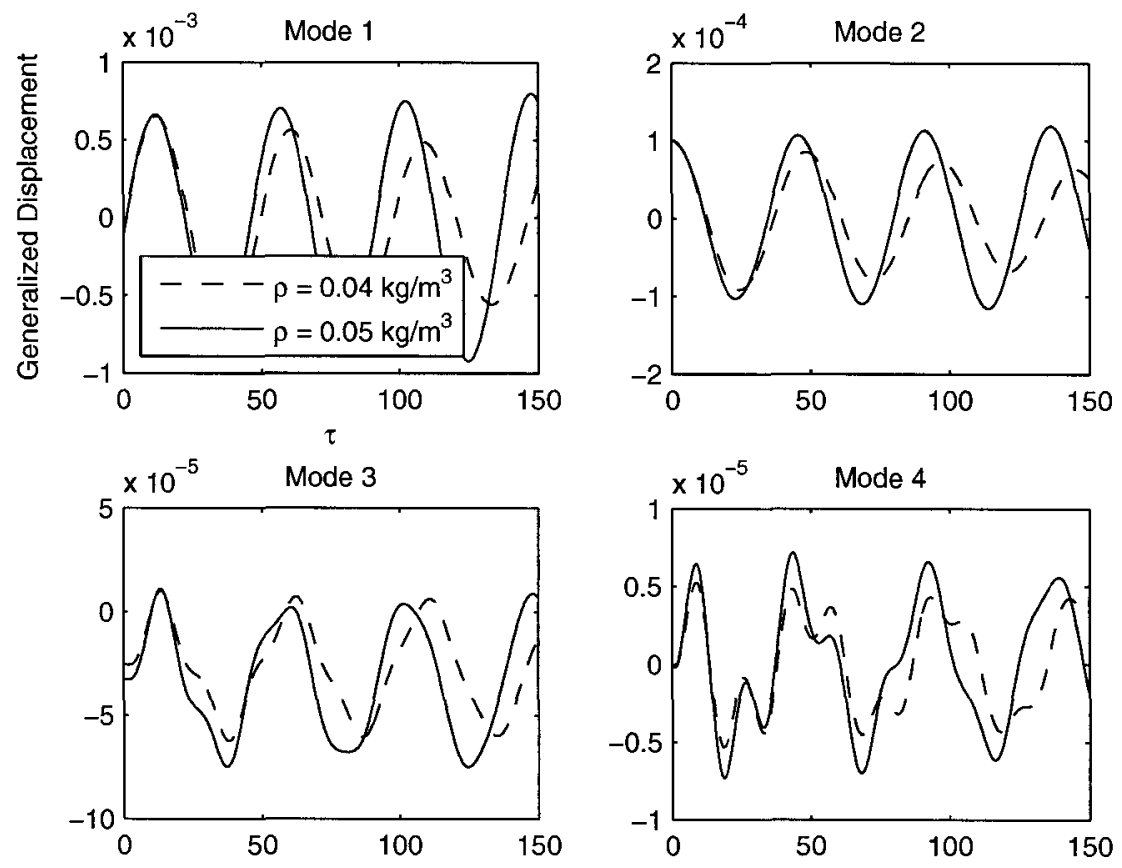

(b) Euler

Figure 6.8: Time marching responses at $M_{\infty}=1.072$. 


\subsection{AIC Correction Method Results}

The unsteady pressure matching correction method, based on a downwash weighting approach, was conducted using KEDLMPL. Unsteady lifting pressures employed for this technique were obtained from Euler and Navier-Stokes CFD simulations undergoing a rigid body pitching motion. These results were employed to calculate the weighting vector, WT. Refer to Chapter 3 for the derivation of the unsteady pressure matching correction method.

Reduced frequencies of the rigid pitching motion were calculated for each Mach number based on experimental flutter speeds and frequencies. Refer to Table 6.2 for the experimental values and the resulting reduced frequencies, $k$. The amplitude of the pitching motion was set to $1^{\circ}$ based on the aeroelastic analysis of Reference [16] for the AGARD 445.6 wing.

The rigid pitch simulations were conducted on a single $3.2 \mathrm{GHz}$ processor. The computational time required using the Euler and Navier-Stokes equations for two rigid pitch cycles was approximately 30 minutes and 45 minutes, respectively.

Table 6.2: Reduced frequencies of the CFD simulations used in the AIC correction method.

\begin{tabular}{cccc}
\hline \hline Mach Number & $U_{\infty}[\mathbf{m} / \mathbf{s}]$ & $\omega_{F}[\mathbf{H z}]$ & $k$ \\
\hline 0.678 & 231.37 & 17.98 & 0.244 \\
0.901 & 296.69 & 16.09 & 0.170 \\
0.960 & 309.01 & 13.89 & 0.141 \\
\hline \hline
\end{tabular}




\subsubsection{Present Work Results}

Unsteady lifting pressures obtained from the Euler and Navier-Stokes rigid pitch simulations were interpolated to mid-span and three-quarter chord for each of the 400 panels contained in the linear aerodynamic model.

Refer to Fig. 6.9 for the unsteady lifting pressures for three spanwise stations. Good agreement between the Euler and Navier-Stokes simulations was observed. The real portion of the unsteady lifting pressures were consistent with the results presented in Reference [16]; however, vast differences were noted for the imaginary portions. The resulting phase angle along the entire wing span was approximately $-45^{\circ}$, thereby suggesting an error in the Fourier transformation. Experimental pressure measurements are unavailable for the AGARD 445.6 wing, thus, a detailed investigation was not conducted to determine the cause of the errors in the unsteady lifting pressures.

The unsteady lifting pressures are employed to calculate the weighting vector, $W T$ for use in the AIC correction procedure. The error in the imaginary portion of these pressures resulted in a highly scaled weighting vector, $W T$. The calculated weighting vector was approximately $10-30 i$ whereas the expected weighting vector is near unity, $1.0 \pm 1.0 i$.

The AIC correction procedure was conducted in KEDLMPL employing the erroneous unsteady lifting pressures obtained from the Navier-Stokes simulations. Refer to Fig. 6.10 for the V-g and V-f plots at $M_{\infty}=0.678$. No coupling between the modes 

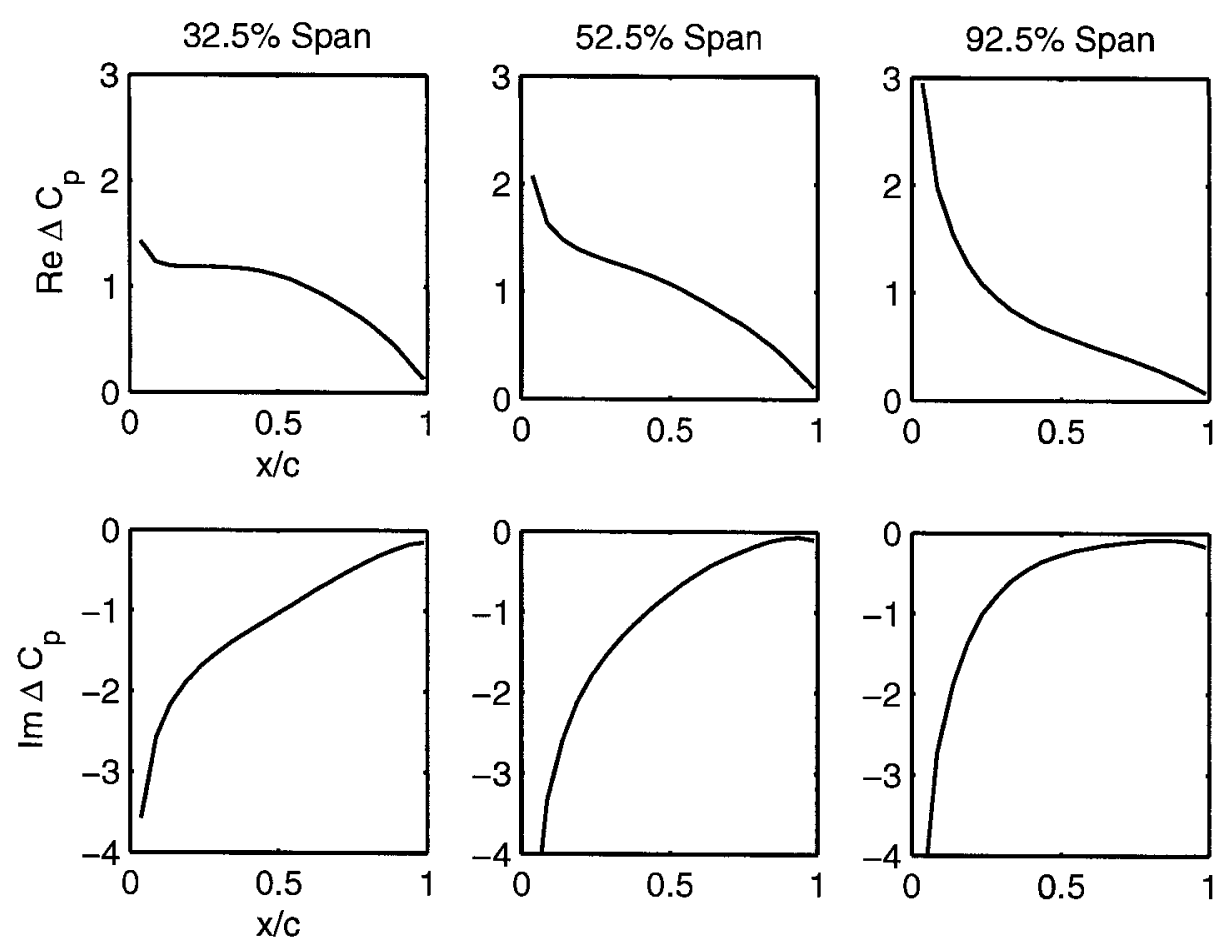

(a) Navier-Stokes
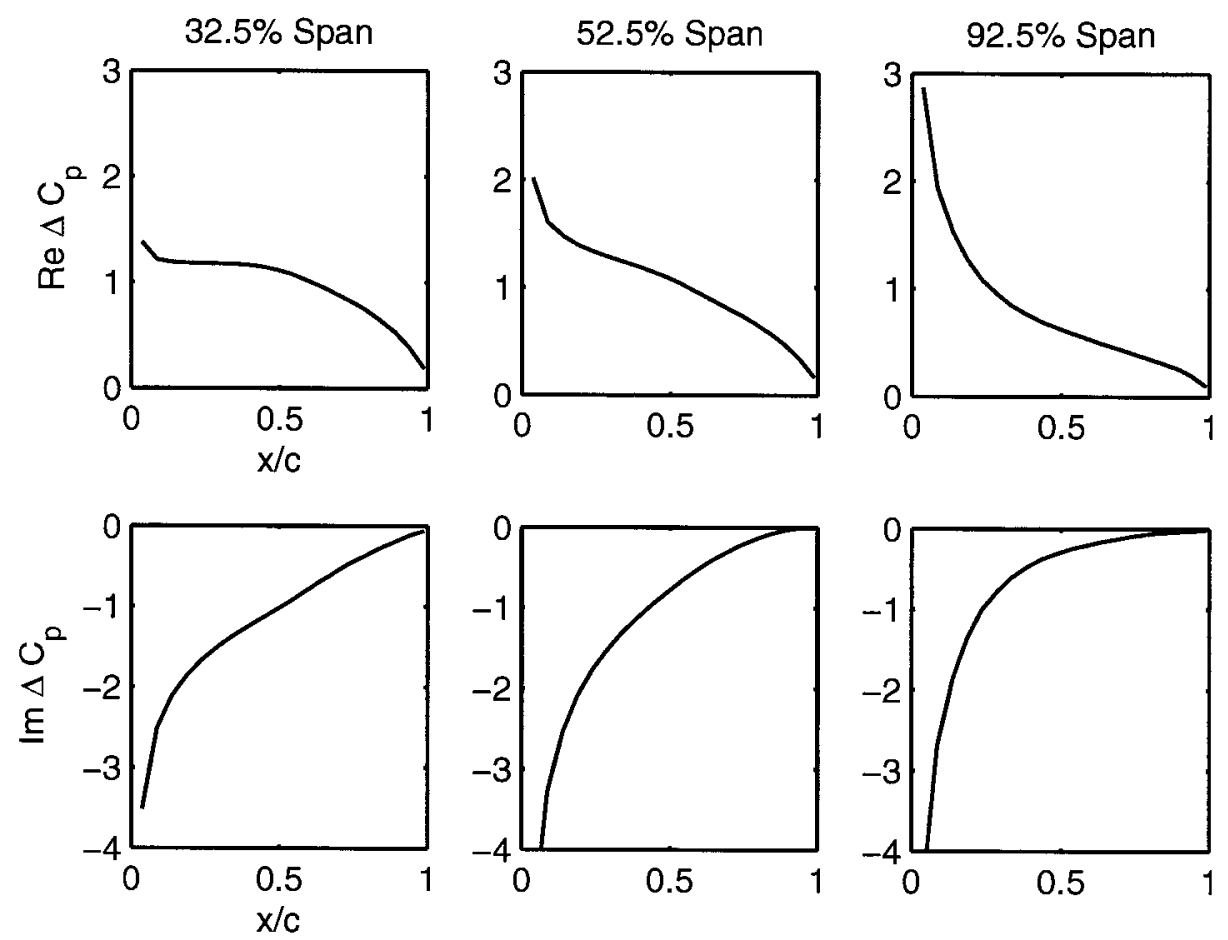

(b) Euler

Figure 6.9: Unsteady $\Delta C_{p}$ over AGARD 445.6 wing at $M_{\infty}=0.678$. 

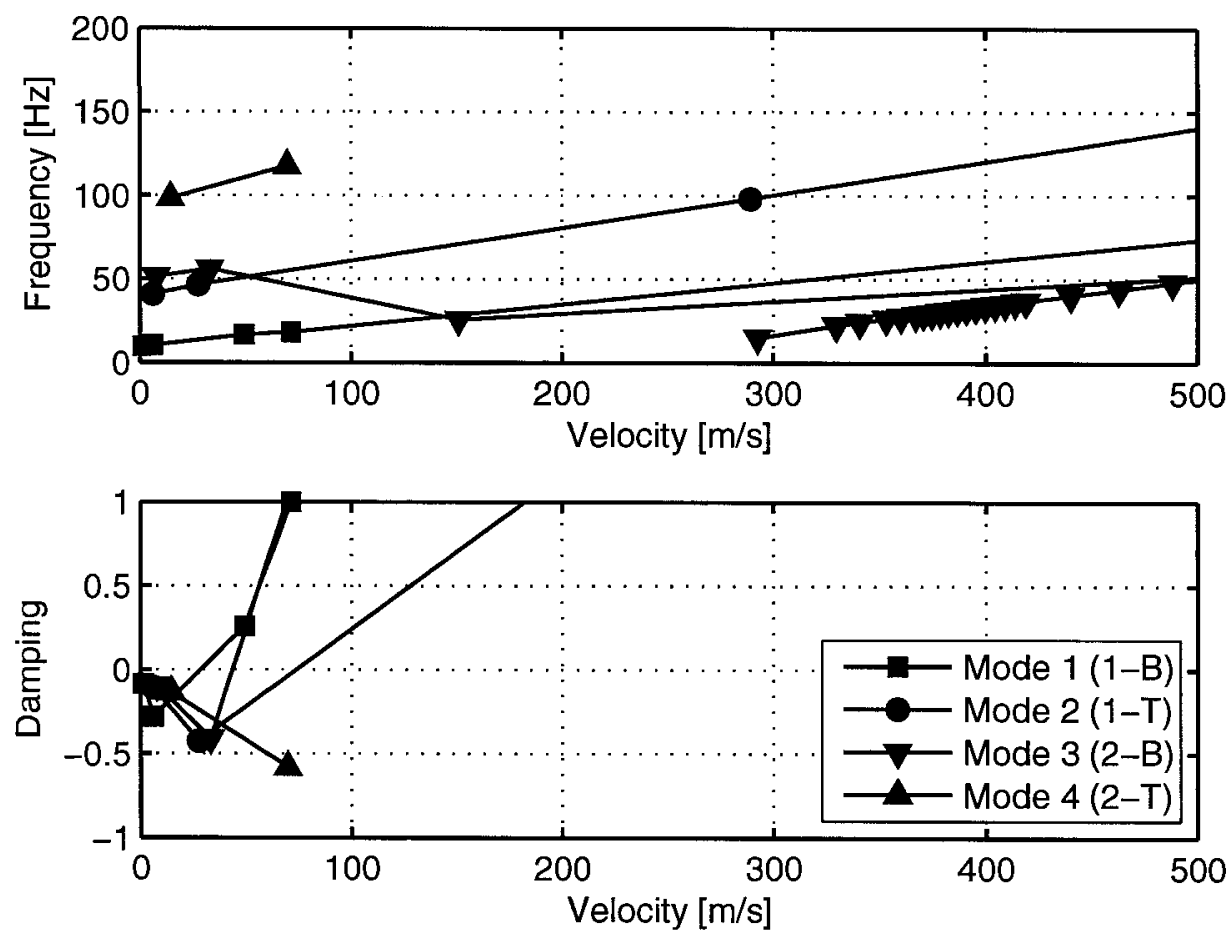

Figure 6.10: AIC corrected frequency domain V-f and V-g plots at $M_{\infty}=0.678$.

was observed in the V-f plot. The resulting flutter speed coefficient was calculated as 0.31, approximately $30 \%$ lower than the experimental, uncorrected frequency domain and time marching results.

As previously discussed, the correction procedure of the present work was developed and successfully implemented in Reference [16]. Therefore, the following subsection will employ the results from Reference [16] in order to illustrate the effectiveness of the correction procedure. 


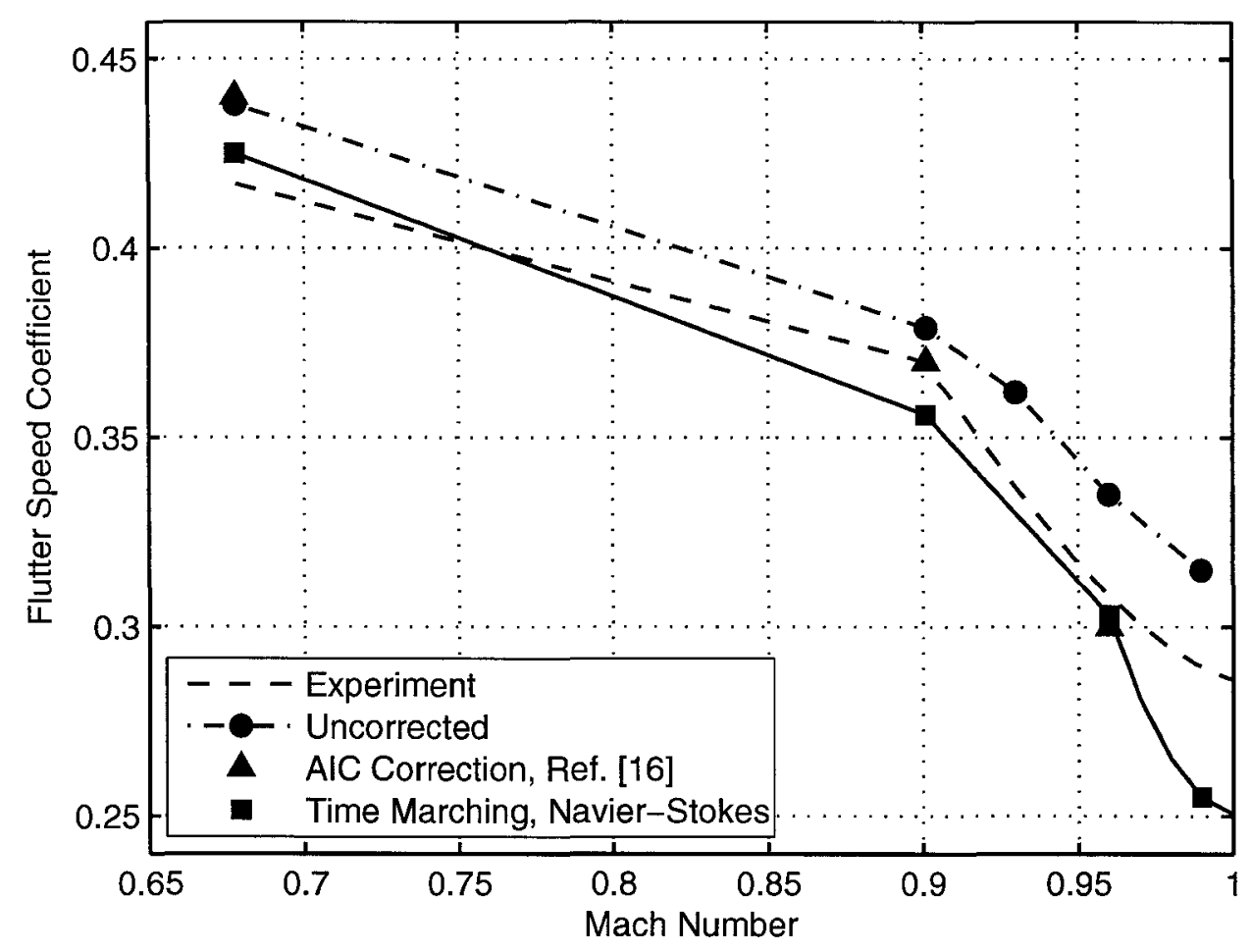

Figure 6.11: Flutter boundaries for AGARD 445.6 wing.

\subsubsection{Referenced Results}

In Reference [16], a flutter analysis was conducted for the AGARD 445.6 wing using the AIC correction procedure for $M_{\infty}=0.678, M_{\infty}=0.901$ and $M_{\infty}=0.960$. The flow was modelled using the Navier-Stokes equations. Similar to the present works, the structural model was based on Reference [53].

Flutter boundaries for the wing are shown in Fig. 6.11. The AIC correction procedure showed good agreement with the experimental and time marching solutions. 


\subsection{Conclusions}

The aircraft industry requires a robust and efficient aerodynamic tool for use in transonic flutter analyses. Presently, a subsonic linearized discrete lifting surface theory, the DLM, is employed but it cannot capture the flow nonlinearities in the transonic regime as illustrated in Fig. 6.2.

These nonlinearities can be captured using two different approaches: (1) performing a time marching simulation using Euler and Navier-Stokes equations; or (2) correcting the DLM aerodynamic data in a frequency domain analysis using CFD results. Refer to Table 6.3 for the comparison of required computational time. Note that the time marching simulations were conducted on a Beowulf cluster consisting of four machines with $3.2 \mathrm{GHz}$ processors. The uncorrected and corrected frequency domain simulations were conducted on a single $2.8 \mathrm{GHz}$ processor.

Table 6.3: Computational time required to calculate each flutter point.

\begin{tabular}{cc}
\hline \hline Method & Time $[\mathrm{hr}]$ \\
\hline KEDLMPL (Uncorrected) & 0.06 \\
MSC/NASTRAN & 0.08 \\
\hline Time Marching, Euler & 4.00 \\
Time Marching, N-S & 6.00 \\
\hline KEDLMPL (AIC Corrected, Euler) & 0.58 \\
KEDLMPL (AIC Corrected, N-S) & 0.83 \\
\hline \hline
\end{tabular}

As illustrated in Fig. 6.3, the Euler and Navier-Stokes time marching simulations 
adequately predicted the transonic flutter boundary. However, the large computational time requirement for this method negates its use in industry. Also, structural design changes occur during most design cycles. The uncorrected and corrected frequency domain approaches produce aerodynamic influence coefficient (AIC) matrices which are independent of the structural model. These matrices can be reapplied to new structural models thus reducing the computational requirements. In contrast, for new structural models, the time marching solutions would require a completely new simulation.

As illustrated in Fig. 6.11, the flutter boundary obtained using the AIC correction frequency domain method demonstrated good agreement with the time marching simulations. The largest error for the AGARD 445.6 wing flutter boundary was $2.8 \%$ at $M_{\infty}=0.901$. Therefore, this approach is ideally suited for industrial applications as this approach: (1) captures the flow nonlinearities in the transonic regime; (2) requires only one-tenth of the computational time of the time marching simulations; and (3) produces AIC matrices which are applicable to new structurals models.

\subsection{Future Works}

The AIC correction method should be further developed once the error in the Fourier transformation has been eliminated from the post-processing of the unsteady lifting pressures. Possible areas to investigate are: (1) correct the AIC matrix using unsteady pressures obtained from modal motions instead of a rigid pitching motion; 
(2) incorporate higher harmonics of the unsteady pressures into the analysis; and (3) extend the method for use on a complete aircraft.

A major limiting factor for future extensions is the lack of experimental data available for validation. A new dataset (MAVRIC-I) of a business jet wing-fuselage flutter model has been generated at NASA Langley Research Center. Unfortunately, however, it has not been released for general use. Ideally, new experimental flutter datasets should contain: (1) aerodynamic unsteady pressure measurements; (2) a detailed structural model; and (3) flutter results in the transonic regime. 


\section{References}

[1] R. L. Bisplinghoff, H. Ashley, and R. L. Halfman, Aeroelasticity. Cambridge, MA: Addison-Wesley Publishing Company Inc., 1955.

[2] I. E. Garrick and W. H. Reed, "Historical development of aircraft flutter," Journal of Aircraft, vol. 18, pp. 898-912, 1981.

[3] A. M. Rampurawala, Assessment of Inter-Grid Transformation for Complete Aircraft Aeroelastic Analysis. Master's thesis, University of Glasgow, 2002.

[4] G. S. L. Goura, Time Marching Analysis of Flutter Using Computational Fluid Dynamics. PhD thesis, University of Glasgow, 2001.

[5] A. Jeziorski, "F-2 wing cracks delay development completion," Flight International, June 16, 1999.

[6] G. Norris, "Asraam flight tests reveal lower-speed F/A-18 flutter," Flight International, October 10, 2000.

[7] M. A. Dornheim, "Report pinpoints factors leading to YF-22 crash," Aviation Week \& Space Technology, pp. 53-54, November 9, 1992.

[8] M. A. Dornheim, "Elevon vibration leads to F-117 crash," Aviation Week 8 Space Technology, p. 30, September 22, 1997.

[9] M. W. Kehoe, "A historical overview of flight flutter testing," NASA-TM-4720, 1995. 
[10] ZONA Technologies Inc., ZAERO Software System - Theoretical Manual - Version 7.0. 2003 .

[11] J. P. Giesing, T. P. Kalman, and W. P. Rodden, "Subsonic unsteady aerodynamics for general configurations, direct application of the non-planar DoubletLattice Method," Air Force Flight Dynamics Lab, Air Force Systems Command, Wright-Patterson Air Force Base, Report AFFDL-TR-71-5 Part 1, 1972.

[12] E. C. Yates Jr., "Modified-strip-analysis method for predicting wing flutter at subsonic to hypersonic speeds," Journal of Aircraft, vol. 3, no. 1, pp. 25-29, 1966.

[13] R. N. Yurkovich, "Status of unsteady aerodynamic prediction for flutter of highperformance aircraft," Journal of Aircraft, vol. 40, no. 5, pp. 832-842, 2003.

[14] E. Albano and W. P. Rodden, "A doublet-lattice method for calculating lift distributions on oscillating surfaces in subsonic flows," AIAA Journal, vol. 7, no. 2, pp. 279-285, 1969.

[15] T. P. Kalman, W. P. Rodden, and J. P. Giesing, "Aerodynamic influence coefficients by the Doublet Lattice Method for interfering nonplanar lifting surfaces oscillating in a subsonic flow," Douglas Report DAC-67977, 1969.

[16] R. G. A. da Silva, A study on correction methods for aeroelastic analysis in transonic flow. PhD thesis, São José dos Campos, 2004.

[17] R. Palacios, H. Climent, A. Karlsson, and B. Winzell, "Assessment of strageies for correcting linear unsteady aerodynamics using CFD or test results," Proc. of the CEAS/AIAA International Forum on Aeroelasticity and Structural Dynamics, Madrid, Spain, pp. 195-210, 2001.

[18] J. P. Giesing, T. P. Kalman, and W. P. Rodden, "Correction factor techniques for improving aerodynamic prediction methods," NASA-CR-144967, 1976. 
[19] R. J. Zwaan, "Verification of calculation methods for unsteady airloads in the prediction of transonic flutter," Journal of Aircraft, vol. 22, no. 10, pp. 833-839, 1985.

[20] D. M. Pitt and C. E. Goodman, "Flutter calculations using Doublet Lattice aerodynamics modified by the Full Potential equations," Proc. of the 28th AIAA/ASME/ASCE/AHS Structures, Structural Dynamics, and Materials Conference, Monterey, California, Paper AIAA-87-0882-CP, 1987.

[21] J. Brink-Spalink and J. M. Bruns, "Correction of unsteady aerodynamic influence coefficients using experimental or CFD data," Proc. of the 41st AIAA/ASME/ASCE/AHS/ASC Structures, Structural Dynamics and Materials Conference and Exhibit, Atlanta, Georgia, Paper AIAA-2000-1498, 2000.

[22] W. P. Rodden and J. D. Revell, "The status of unsteady aerodynamic influence coefficients," Fairchild Fund Paper FF-33, 1962.

[23] H. Bergh and R. J. Zwaan, "A method for estimating unsteady pressure distributions for arbitrary vibration modes from theory and from measured distributions for one single mode," NLR-TR F.250, National Aerospace Laboratory, Netherlands, 1966.

[24] W. Luber and H. Schmid, "Flutter investigations in the transonic flow regime for a fighter type aircraft," AGARD Report No. 703, 1982.

[25] M. L. Baker, "CFD based corrections for linear aerodynamic methods," AGARD Report No. 822, 1997.

[26] I. Jadic, D. Hartley, and J. Giri, "An enhanced correction factor technique for aerodynamic influence coefficient methods," Proc. of MacNeal-Schwendler Corporation's Aerospace User's Conference, 1999. 
[27] I. Jadic, D. Hartley, and J. Giri, "Improving the linear aerodynamic approximation in linear aeroelasticity," Proc. of the 41st AIAA/ASME/ASCE/AHS/ASC Structures, Structural Dynamics and Materials Conference and Exhibit, Atlanta, Georgia, Paper AIAA-2000-1450, 2000.

[28] H. C. Garner, "A practical framework for evaluation of oscillating aerodynamic loadings on wings in supercritical flows," AE Technical Memorandum Structures 900, United Kingdom, 1977.

[29] K. Dau, "A semi-empirical method for calculating pressures on oscillating wings in unsteady transonic flow," Report No. DA-EF24-B08/92, Deutche Airbus, 1992.

[30] G. SenGupta, "Evaluation of the Dau-Garner method for predicting unsteady pressures in transonic flow," Proc. of the International Symposium on Aeronautical Science \& Technology, Jakarta, Indonesia, Paper ISASTI-96-1.3.4, 1996.

[31] K. Yonemoto, "A practical method predicting transonic wing flutter phenomena," Proc. of the 14th International Council of the Aeronautical Sciences Congress, Toulouse, France, pp. 724-732, 1984.

[32] D. D. Liu, Y. F. Kao, and K. Y. Fung, "An efficient method for computing unsteady transonic aerodynamics of swept wings with control surfaces," Journal of Aircraft, vol. 25, no. 1, pp. 25-31, 1988.

[33] P. C. Chen, D. Sarhaddi, and D. D. Liu, "Transonic aerodynamic influence coefficient approach for aeroelastic and MDO applications," Journal of Aircraft, vol. 37, no. 1, pp. 85-94, 2000.

[34] P. Guezaine, C. Farhat, and G. Brown, "Application of three field nonlinear fluid-structure formulation to the prediction of aeroelastic parameters of an F-16 fighter," Computers and Fluids, vol. 32, pp. 3-29, 2003. 
[35] C. J. Borland and D. P. Rizetta, "Nonlinear transonic flutter analysis," $A I A A$ Journal, vol. 20, no. 11, pp. 1606-1615, 1982.

[36] J. T. Batina, H. J. Cunningham, and R. M. Bannett, "Modern wing flutter analysis by computational fluid dynamics methods," Journal of Aircraft, vol. 25, no. 10, pp. 962-968, 1988.

[37] E. M. Lee-Rausch and J. T. Bantina, "Calculation of AGARD 445.6 wing flutter using Navier-Stokes aerodynamics," Proc. of the AIAA Applied Aerodynamics Conference, Monterey, California, Paper AIAA-93-3476-CP, 1993.

[38] G. P. Guruswamy and C. Byun, "Direct coupling of Euler flow equations with plate finite element structures," AIAA Journal, vol. 33, no. 2, pp. 375-377, 1994.

[39] R. Melville, "Nonlinear simulation of F-16 aeroelastic instability," Proc. of the 39th AIAA Aerospace Sciences Meeting and Exhibit, Reno, Nevada, Paper AIAA-2001-0570, 2001.

[40] K. J. Badcock, M. A. Woodgate, F. Cantariti, and B. E. Richards, "Solution of the unsteady euler equations in three dimensions using a fully unfactored method," Aerospace Engineering Report 9909, University of Glasgow, 1999.

[41] W. P. Rodden, "A comparison of methods used in interfering lifting surface theory," AGARD Report No. 643, 1976.

[42] E. Sulaeman, Effect of Compressive Force on Aeroelastic Stability of a StrutBraced Wing. PhD thesis, Virginia Polytechnic Institute and State University, 2001.

[43] K. J. Badcock, B. E. Richards, and M. A. Woodgate, "Elements of computational fluid dynamies on block structured grids using implicit solvers," Progress in Aerospace Sciences, vol. 36, pp. 351-392, 2000. 
[44] M. A. Woodgate, K. J. Badcock, A. M. Rampurawala, and B. Richards, "Aeroelastic calculations for the hawk aircraft using the euler equations," Aerospace Engineering Report 0313, University of Glasgow, 2003.

[45] G. L. Davis, Trailing Edge Flap Control of Dynamic Stall on Helicopter Rotor Blades. Master's thesis, Carleton University, 2005.

[46] C. R. Group, "Modelling of turbulence in eros-uk," Doc. No. TN02-012, University of Glasgow, 2002.

[47] H. Tijdeman and J. W. G. van Nunen, "Transonic wind-tunnel tests on an oscillating wing with external store, part 1: General description," NLR TR 78105 U Part 1, 1978.

[48] J. W. G. van Nunen and H. Tijdeman, "Results of transonic wind tunnel measurements on an oscillating wing with external store (data report)," NLR TR $78030 \mathrm{U}, 1978$.

[49] K. J. Badcock, M. A. Woodgate, and B. Richards, "Direct aeroelastic bifurcation analysis of a symmetric wing based on the euler equations," Aerospace Engineering Report 0315, University of Glasgow, 2003.

[50] R. E. Gordnier and R. B. Melville, "Transonic flutter simulations using an implicit aeroelastic solver," Journal of Aircraft, vol. 37, no. 5, pp. 872-879, 2000.

[51] E. C. Yates Jr., AGARD Standard Aeroelastic Configurations for Dynamic Response I-Wing 445.6. AGARD Report 765, 1988.

[52] W. P. Rodden and E. H. Johnson, MSC/NASTRAN Aeroelastic Analysis User's Guide. Los Angeles, CA: The MacNeal-Schwendler Corporation, Version 68, 1994. 
[53] R. M. Kolonay, Unsteady Aeroelastic Optimization in the Transonic Regime. PhD thesis, Purdue University, 1996.

[54] K. Kavukcuolu, Wing Flutter Analysis with an Uncoupled Method. Master's thesis, Middle East Technical University, 2003. 